\title{
CRISPR/CAS9 based engineering of actinomycetal genomes
}

\author{
Weber, Tilmann; Tong, Yaojun; Lee, Sang Yup
}

Publication date:

2016

Document Version

Publisher's PDF, also known as Version of record

Link back to DTU Orbit

Citation (APA):

Weber, T., Tong, Y., \& Lee, S. Y. (2016). CRISPR/CAS9 based engineering of actinomycetal genomes. (Patent No. WO2016150855).

\section{General rights}

Copyright and moral rights for the publications made accessible in the public portal are retained by the authors and/or other copyright owners and it is a condition of accessing publications that users recognise and abide by the legal requirements associated with these rights.

- Users may download and print one copy of any publication from the public portal for the purpose of private study or research.

- You may not further distribute the material or use it for any profit-making activity or commercial gain

- You may freely distribute the URL identifying the publication in the public portal

If you believe that this document breaches copyright please contact us providing details, and we will remove access to the work immediately and investigate your claim 
(12) INTERNATIONAL APPLICATION PUBLISHED UNDER THE PATENT COOPERATION TREATY (PCT)

(19) World Intellectual Property Organization

International Bureau

(43) International Publication Date

29 September $2016(29.09 .2016)$

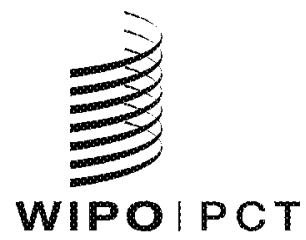

|||||| |||||||||||||||||||||||||||||||||||||||||||||||||||||||||||||||||||||||||||||||||||||||||

(10) International Publication Number

WO 2016/150855 A1

(51) International Patent Classification:

C12N 15/63 (2006.01) C12N 15/90 (2006.01)

(21) International Application Number:

PCT/EP2016/055967

(22) International Filing Date:

18 March $2016(18.03 .2016)$

(25) Filing Language:

English

(26) Publication Language:

English

(30) Priority Data: 15160126.7

20 March 2015 (20.03.2015)

EP

(71) Applicant: DANMARKS TEKNISKE UNIVERSITET [DK/DK]; Anker Engelunds Vej 1, Bygning 101A, 2800 Kgs. Lyngby (DK).

(72) Inventors: WEBER, Tilmann; Slotsgade 8F, st.tv., 3480 Fredensborg (DK). TONG, Yaojun; Kammerrådensvej 31, st.tv., 2970 Hørsholm (DK). LEE, Sang Yup; Expo Apt. 212-702, Expo-ro 448, Yuseong-gu, Daejeon 305-761 (KR).

(74) Agent: HØIBERG A/S; Adelgade 12, 1304 Copenhagen $\mathrm{K}$ (DK).

(81) Designated States (unless otherwise indicated, for every kind of national protection available): $\mathrm{AE}, \mathrm{AG}, \mathrm{AL}, \mathrm{AM}$, $\mathrm{AO}, \mathrm{AT}, \mathrm{AU}, \mathrm{AZ}, \mathrm{BA}, \mathrm{BB}, \mathrm{BG}, \mathrm{BH}, \mathrm{BN}, \mathrm{BR}, \mathrm{BW}, \mathrm{BY}$, $\mathrm{BZ}, \mathrm{CA}, \mathrm{CH}, \mathrm{CL}, \mathrm{CN}, \mathrm{CO}, \mathrm{CR}, \mathrm{CU}, \mathrm{CZ}, \mathrm{DE}, \mathrm{DK}, \mathrm{DM}$,
DO, DZ, EC, EE, EG, ES, FI, GB, GD, GE, GH, GM, GT, HN, HR, HU, ID, IL, IN, IR, IS, JP, KE, KG, KN, KP, KR, KZ, LA, LC, LK, LR, LS, LU, LY, MA, MD, ME, MG, MK, MN, MW, MX, MY, MZ, NA, NG, NI, NO, NZ, OM, PA, PE, PG, PH, PL, PT, QA, RO, RS, RU, RW, SA, SC, SD, SE, SG, SK, SL, SM, ST, SV, SY, TH, TJ, TM, TN, TR, TT, TZ, UA, UG, US, UZ, VC, VN, ZA, ZM, ZW.

(84) Designated States (unless otherwise indicated, for every kind of regional protection available): ARIPO (BW, GH, GM, KE, LR, LS, MW, MZ, NA, RW, SD, SL, ST, SZ, TZ, UG, ZM, ZW), Eurasian (AM, AZ, BY, KG, KZ, RU, TJ, TM), European (AL, AT, BE, BG, CH, CY, CZ, DE, DK, EE, ES, FI, FR, GB, GR, HR, HU, IE, IS, IT, LT, LU, LV, MC, MK, MT, NL, NO, PL, PT, RO, RS, SE, SI, SK, SM, TR), OAPI (BF, BJ, CF, CG, CI, CM, GA, GN, GQ, GW, KM, ML, MR, NE, SN, TD, TG).

Declarations under Rule 4.17:

- of inventorship (Rule 4.17(iv))

Published:

- with international search report (Art. 21(3))

- before the expiration of the time limit for amending the claims and to be republished in the event of receipt of amendments (Rule 48.2(h))

- with sequence listing part of description (Rule 5.2(a))

(54) Title: CRISPR/CAS9 BASED ENGINEERING OF ACTINOMYCETAL GENOMES

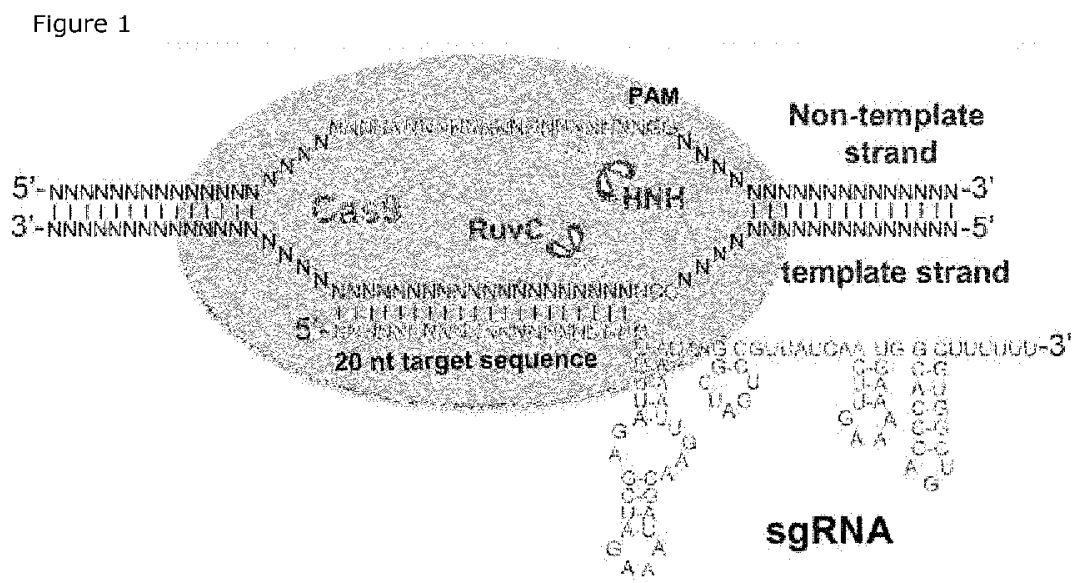

(57) Abstract: The present invention relates to CRISPR/Cas-based methods for generating random-sized deletions around at least one target nucleic acid sequence, or for generating precise indels around at least one target nucleic acid sequence, or for modulating ranscription of at least one target nucleic acid sequence. Also disclosed is a clonal library comprising clones with random-sized de letions, as well as polynucleotides, polypeptides, cells and kits useful for performing the present methods. The present methods can be performed in organisms where gene editing is typically considered as difficult, such as actinomycetes, in particular streptomycetes. 
CRISPR/CAS9 BASED ENGINEERING OF ACTINOMYCETAL GENOMES

\section{Field of invention}

5

The present invention relates to CRISPR/Cas-based methods for generating randomsized deletions around at least one target nucleic acid sequence, or for generating precise indels around at least one target nucleic acid sequence, or for modulating transcription of at least one target nucleic acid sequence. Also disclosed is a clonal library comprising clones with random-sized deletions, as well as polynucleotides, polypeptides, cells and kits useful for performing the present methods. The present methods can be performed in organisms where gene editing is typically considered as difficult, such as actinomycetes, in particular streptomycetes.

\section{Background of invention}

Actinomycetes are Gram-positive bacteria with the capacity to produce a wide variety of medically and industrially relevant secondary metabolites, including many antibiotics, herbicides, parasiticides, anti-cancer agents, and immunosuppressants. It becomes

20 harder and harder to find new bioactive compounds from actinomycetes using traditional approaches.

Recent advances in genome sequencing and genome mining have significantly accelerated the ability to identify secondary metabolism genes and gene clusters. Precise gene editing technologies are needed to enable systematic reverse engineering of causal genetic variations by allowing selective perturbation of individual genetic elements, as well as to advance synthetic biology and biotechnology. There are four major universal gene editing tools developed so far: 1) meganucleases derived from microbial mobile genetic elements, 2) zinc finger (ZF) nucleases based on eukaryotic transcription factors, 3) transcription activator-like effectors (TALEs) from Xanthomonas bacteria, and 4) the RNA-guided DNA endonuclease Cas9 from the type II bacterial adaptive immune system Clustered Regularly Interspaced Short Palindromic Repeats (CRISPR), called CRISPR-Cas9 system. However, each of the first three methods has its own unique limitations: the specificity of a meganuclease for a target DNA is difficult to control, the assembly of functional zinc finger proteins with the desired DNA binding 
specificity remains a major challenge, and the construction of novel TALE arrays are labour intensive and costly.

The CRISPR-Cas9 system displays certain advantages. The CRISPR nuclease Cas9 can be guided by a short single guide RNA (sgRNA) that recognizes the target DNA via Watson-Crick base pairing (Figure $1 \mathrm{~A}$ ) instead of complex protein-DNA recognition, thereby easing the design and construction of targeting vectors. The sgRNAs are artificially generated chimeras of the CRISPR RNA (crRNA) and the associated transactivating CRISPR RNA (tracrRNA) found in the native CRISPR systems, which origi-

10 nally corresponds to phage sequences, constituting the natural mechanism for CRISPR antiviral defense of bacteria and archaea, but can be easily replaced by a sequence of interest to reprogram the Cas9 nuclease for gene editing. Multiplexed targeting by Cas 9 can now be achieved at an unprecedented scale by introducing a plurality of sgRNAs rather than a library of large, bulky proteins.

The Cas 9 protein family is characterized by two signature nuclease domains, $\mathrm{HNH}$ and RuvC. A critical feature of recognition by CRISPR-Cas9 is the protospacer-adjacent motif (PAM), which flanks the 3' end of the DNA target site (Figure 1) and directs the DNA target recognition by the Cas9-sgRNA complex. The Cas9 and the sgRNA first 20 form a complex, and the complex subsequently starts to scan the whole genome for the PAM sequences. Once the complex has identified the PAM, which can have on its 5' flank a sequence complementary to the target sequence within the sgRNA in the complex, the complex binds to this position. This triggers the Cas 9 nuclease activity by activating the $\mathrm{HNH}$ and RuvC domains.

The CRISPR/Cas9 system generates a break, such as a nick or a double-strand break (DSB) in the DNA, which is repaired by one of the two main repair pathways: nonhomologous end-joining (NHEJ) or homologous recombination (HR). HR requires the presence of a homologous template DNA, which can comprise additional sequences which can thus be introduced at the site of the break. NHEJ does not require the presence of donor DNA, and usually results in small deletions. The system can thus be used for integrating new sequences into a target sequence, or for the precise generation of deletions around the target site. 
Because of its modularization and easy handling, the CRISPR-Cas9 system has been successfully applied as a gene editing tool in a wide range of organisms such as Saccharomyces cerevisiae, some plants, Caenorhabditis elegans, Drosophila, Chinese hamster ovary $(\mathrm{CHO})$ cells, frogs, mice, rats, rabbits, and human cells with high specificity. Recently, the CRISPR-Cas9 system was re-programmed to control gene expression by mutating the $\mathrm{HNH}$ and RuvC domains of Cas9 (D10A and $\mathrm{H} 840 \mathrm{~A}$ ), resulting in a catalytically dead Cas 9 (dCas9) lacking endonuclease activity. This system has so far successfully been applied in Escherichia coli (Qi, L. S., et, al. 2013).

10 As stated above, one of the challenges in the deep application of actinomycetes is to systematically engineer them for the overproduction of effective secondary metabolites and non-natural chemical compounds as well as new bioactive compounds, which corresponds to a fundamental objective of metabolic engineering. Unfortunately, genetic manipulation of actinomycetes is considered to be more difficult than model organisms,

15 such as Escherichia coli and Saccharomyces cerevisiae. This is due in part to their more diverse genomic contents; for example, the GC content of their genomes is high.

There are to our knowledge only two very recent publications describing a CRISPR based system using homologous recombination templates to generate defined muta20 tions in streptomycetes (Cobb et al., 2014, Huang et al., 2015). The use of CRISPRbased systems for generating random-sized, targeted deletions around a target site has not yet been reported.

Thus, rapid, efficient and convenient methods for gene editing of actinomycetes, in particular for streptomycetes, are needed.

\section{Summary of invention}

30 The invention is as defined in the claims.

Herein are disclosed methods useful for gene editing. These methods are based on the surprising finding that in organisms having a partly deficient non-homologous endjoining pathway (NHEJ), gene editing based on the CRISPR/Cas 9 system targeting a

35 nucleic acid sequence of interest results in the generation of clones with random-sized deletions around the target site. In order to generate precise indels (i.e. precise inser- 
tions or deletions) around a target site in such organisms, the NHEJ pathway can be restored by engineering the host cell so that it has a fully functional NHEJ pathway.

The methods described herein are of particular interest for organisms where gene editing is typically considered to be labor-intensive, such as actinomycetes. The methods can be used to generate clonal libraries in order to investigate a given pathway, for example in order to optimize production of a secondary metabolite.

Also described herein is a method for modulating transcription of a nucleic acid sequence of interest by using a catalytically dead Cas9. This method can be applied to actinobacteria, e.g. streptomycetes.

\section{Description of Drawings}

Figure 1. Diagram of the Cas 9 and sgRNA complex. The Cas9 HNH and RuvC-like domains each cleave one strand of the sequence targeted by the sgRNA; the trinucleotide PAM is labelled; the binding of the $20 \mathrm{nt}$ target sequence to the genome is shown; the sgRNA core structure and sequence is shown.

Figure 2. Design of easily changeable sgRNA scaffold: the forward primer, labelled as "P-F", comprises a $20 \mathrm{nt}$ sgRNA core sequence, a $20 \mathrm{nt}$ target sequence and the Ncol sequence, while the reverse primer, labelled as "P-R", comprises a $20 \mathrm{nt}$ sgRNA core sequence and the SnaBI sequence. To construct a new sgRNA, a $20 \mathrm{nt}$ target sequence of interest is designed and integrated in the forward primer. The arrow represents the ermE* promoter, while the circle represents the to terminator, and the core sgRNA is shown as a box.

Figure 3. Map of pCRISPR-Cas9. Restriction endonuclease sites are available for additional elements sub-cloning, for instance, the Stul site.

Figure 4. Actinorhodin biosynthesis. A. Organization of the actinorhodin biosynthetic gene cluster; B. The steps to synthetize actinorhodin are: I. 1X Acetyl-CoA and 7x malonyl-CoA are condensed to form the carbon skeleton by Actl; II. The above carbon backbone is cyclized to form a three ring intermediate, DNPA by ActIII, ActVII, ActlV, ActVI-1 and ActVI-3; III. DNPA is then modified to form DHK by ActVI-2, ActVI-4 and 
ActVA-6; IV. 2 DHK is dimerized to form the final product, actinorhodin by ActVA-5 and ActVB. The arrows mark the two selected genes.

Figure 5. Functional sgRNAs PCR screening results: the positive size is $234 \mathrm{bp}$, the negative size is $214 \mathrm{bp}$, the agrose gel concentration is $4 \%$ in TAE. A-C, 36 clones for actIORF1 gene; D-F, 36 clones for actVB gene.

Figure 6. Actinorhodin biosynthetic pathway was inactivated by CRISPR-Cas9. 1-5, represent strains WT, $\Delta$ actlorf1-1, Mismatch, $\Delta a c t v b-1$, and No Target, respectively; the plate in the left panel is without inducer thiostrepton, while the plate in the right panel is with inducer thiostrepton, the $\mathrm{pH}$ of the plates is $>7$. A. ISP2 plate without antibiotics. All five strains are blue. B. ISP2 plate with $1 \mu \mathrm{g} / \mathrm{ml}$ thiostrepton. Labels correspond to those in B. The blue from strains $\Delta a c t l o r f 1-1$ and $\Delta a c t v b-1$ disappeared. The photos were taken after 7 days incubation at $30^{\circ} \mathrm{C}$.

Figure 7. Actinorhodin detection by UV-visible spectrometry. When the $\mathrm{pH}$ is lowered to 2, actinorhodin turns from blue to red, and has a maximum absorption at about $530 \mathrm{~nm}$. From the scanning, the actinorhodin peak of $\Delta a c t l o r f 1$ and $\Delta a c t v b$ disappeared.

Figure 8. Analysis of the sequencing data. A. Heatmap of the 7 mapped sequencing samples to the $S$. coelicolor A3(2) reference genome. Dark colours represent a high read coverage, white represents low/no coverage. Displayed is the region spanning 5508800 to 5557230 of the S. coelicolor genome. The actinorhodin gene cluster is denoted by brackets; the target sites of the actIORF1 and actVB sgRNAs are displayed as arrows. The deletion sizes are shown on the map. 1-7 represent strains: WT, No Target, Mismatch, $\Delta a c t / o r f 1-1, \Delta a c t / o r f 1-2, \Delta a c t v b-1$, and $\Delta a c t v b-2$, respectively. B. Alignment of the sequence traces of $\Delta a c t l o r f 1-1$ with the WT. The arrow indicates the genomic target site of the sgRNA: Actlorf1-6 T. The PAM sequence is shown. C. and D. DNA sequences of 8 randomly selected clones without actinorhodin production aligned to the WT genomic sequence of actIORF1 and actVB, respectively. The arrow indicates the genomic target sites of the related sgRNAs. The PAM sequences are shown. Dark shadow, light shadow with a dash and dark shadow with a box indicate insertions, deletions and substitutions, respectively. 
Figure 9. Plasmid map for pCRISPR-Cas9-ScaligD. An expression cassette of S. carneus ligD was introduced into pCRISPR-Cas9 using Gibson Assembly in Stul site. The S. carneus ligD was under control by ermE* promoter, ending with a to terminator.

Figure 10. HDR pathway to repair the DNA DSBs caused by CRISPR-Cas9 system. A. and $B$. Diagrams of the CRISPR-Cas 9 vectors with homologous recombination templates for act/ORF1 and actVB. C. and D. Colony PCR of 10 randomly selected clones that lost actinorhodin production to confirm deletion of actIORF1 (C) and actVB (D) after use of the two vectors in A and B. I, II, and III represent the WT genome, ac10 tIORF1 deleted and actVB deleted genome, respectively. 1-10 represent 10 randomly selected clones that lost actinorhodin production.

Figure 11. The plasmid map for pCRISPR-dCas9. The only difference between pCRISPR-dCas 9 and pCRISPR-Cas 9 is the Cas 9 was a catalytically dead version without the endonuclease activity (D10A and H840A), called dCas9 in pCRISPRdCas9.

Figure 12. CRISPRi effectively silences actIORF1 expression in a reversible manner. A. Location of the twelve sgRNAs for CRISPRi. Half were designed to target the promoter region, while the other half were designed to target the ORF. In addition, half target the template strand and half target the non-template strand. The dashes represent sgRNAs. B. $530 \mathrm{~nm}$ absorbance of extracts from cultures tested with the twelve sgRNAs shown in A relative to the wild-type control. Left panel shows the sgRNAs target on promoter region, while right panel shows the sgRNAs target on ORF region. Mean values from three independent extractions are shown. Error bars represent the standard deviation from three independent extractions. C. and D. Reversibility of the CRISPRi system. Red clones become blue when the incubation temperature is increased to $37^{\circ} \mathrm{C}$, indicating that the CRISPRi effect has gone away. The red color is boxed, while the blue is not. 0-12 represent sgRNAs: control (without any sgRNA), orf1p-A1 NT, orf1p-A4 NT, orf1p-A5 NT, orf1p-S1 T, orf1p-S3 T, orf1p-S5 T, Actlorf1-1 NT, Actlorf1-7 NT, Actlorf1-8 NT, Actlorf1-2 T, Actlorf1-3 T, and Actlorf1-4 T, respectively. 


\section{Detailed description of the invention}

The present inventors have surprisingly found that a partial deficiency of the nonhomologous end-joining (NHEJ) pathway in a host cell conferred the host cell interesting properties. For example, inducing a CRISPR-Cas9 system in said host cell results in the generation of random-sized deletions around a target site recognized by said CRISPR-Cas 9 system. On the other hand, restoring full functionality of the NHEJ pathway prior to or simultaneously with induction of the CRISPR-Cas9 system results in the generation of precise indels around the target site.

10

In a first aspect, the invention relates to a method for generating at least one deletion around at least one target nucleic acid sequence comprised within a host cell having a non-homologous end-joining (NHEJ) pathway which is at least partly deficient, said method comprising the steps of:

(i) optionally, restoring the full functionality of the NHEJ pathway,

(ii) inducing a CRISPR-Cas9 system in said host cell, wherein said CRISPR-Cas 9 system is able to generate at least one break in said at least one target nucleic acid sequence and wherein the CRISPRCas9 system comprises a Cas9 nuclease and at least one guiding means,

thereby generating:

a. if the method does not comprise step (i)., at least one random-sized deletion around said at least one target nucleic acid sequence, wherein said at least one deletion is a random-sized deletion of at least $1 \mathrm{bp}$; or

b. if the method does comprise step (i), at least one indel around said at least one target nucleic acid sequence, wherein said at least one indel is a deletion or insertion of at least $1 \mathrm{bp}$.

In a second aspect, the invention relates to a polynucleotide having at least $94 \%$ identity with SEQ ID NO: 1 , such as at least $95 \%$ identity, such as at least $96 \%$ identity, such as at least $97 \%$ identity, such as at least $98 \%$ identity, such as at least $99 \%$ identity, such as $100 \%$ identity with SEQ ID NO: 1. 
In yet another aspect, the invention relates to a polypeptide encoded by the polynucleotide described herein.

In yet another aspect, the invention relates to a cell comprising the polynucleotide described herein.

In yet another aspect, the invention relates to a cell comprising the polypeptide described herein.

10 In yet another aspect, the invention relates to a vector comprising the polynucleotide described herein.

In yet another aspect, the invention relates to a clonal library obtainable by the above method, said clonal library comprising a plurality of clones harboring at least one deletion and/or indel around at least one target nucleic acid sequence, wherein said deletion is a random-sized deletion of at least $1 \mathrm{bp}$ and wherein said indel is a deletion or insertion of at least $1 \mathrm{bp}$.

In yet another aspect, the invention relates to a method for selectively modulating transcription of at least one target nucleic acid sequence in a host cell, the method comprising introducing into the host cell:

i. at least one guiding means, or a nucleic acid comprising a nucleotide sequence encoding guiding means, wherein the guiding means comprises a nucleotide sequence that is complementary to a target nucleic acid sequence in the host cell; and

ii. a variant Cas9, or a nucleic acid comprising a nucleotide sequence encoding the variant Cas9, wherein the variant Cas9 is the polypeptide described herein, or wherein the nucleotide sequence encoding the variant Cas 9 is the polynucleotide described herein, and wherein the variant Cas 9 has reduced endodeoxyribonuclease activity, wherein said guiding means and said variant Cas 9 form a complex in the host cell, said complex selectively modulating transcription of at least one target nucleic acid in the host cell. 
In yet another aspect, the invention relates to a clonal library obtainable by the methods disclosed herein, said clonal library comprising a plurality of clones harbouring at least one deletion and/or indel around at least one target nucleic acid sequence, wherein said deletion is a random-sized deletion of at least $1 \mathrm{bp}$ and wherein said indel is a deletion or insertion of at least $1 \mathrm{bp}$.

In yet another aspect, the invention relates to a kit for performing the method of the first aspect, said kit comprising a vector comprising a nucleic acid sequence encoding a Cas9 nuclease or a variant thereof, and instructions for use.

10

In yet another aspect, the invention relates to a kit for performing the method of the second aspect, said kit comprising a vector comprising a variant Cas9, or a nucleic acid comprising a nucleotide sequence encoding the variant Cas9, wherein the variant Cas 9 is the polypeptide of claim 4 or the nucleotide sequence encoding the variant Cas 9 is the polynucleotide of claim 3 , and wherein the variant Cas9 has reduced endodeoxyribonuclease activity, and instructions for use.

\section{Definitions}

Break: the term 'break' shall be construed as referring to a double strand break, a 20 single strand break or a nick in a DNA strand.

Cluster or gene cluster: these terms refer to a group of closely linked genes that are collectively responsible for a multi-step process such as the biosynthesis of a metabolite, for example a secondary metabolite.

CRISPR-Cas9 system: the terms 'CRISPR-Cas9', 'CRISPR/Cas9' and 'type II CRISPR' and systems thereof will be used interchangeably and refer to a system comprising a CRISPR-Cas9 protein and at least one guiding means, so that the CRISPR-Cas9 system is capable, when induced, of generating at least one break in at least one target nucleic acid sequence. Thus a CRISPR-Cas9 system herein comprises Cas9 and at least one guiding means. The guiding means are as defined below.

Deletion: the term 'deletion' refers to the deletion of one or more nucleotides or base pairs in a nucleic acid sequence. The term 'precise deletion' refers to smaller deletions, while the term 'random-sized deletion' refers to deletions of at least $1 \mathrm{bp}$ which can 
span over several kilobases, as detailed below.

Double strand break (DSB): a double strand break (DSB) as understood herein refers to a break on both strands of a nucleic acid. DSBs are particularly hazardous to the cell because they can lead to genome rearrangements. Two major mechanisms exist to repair DSBs: non-homologous end joining (NHEJ) and homologous recombination (HR). The choice of pathway depends on parameters such as the nature of the organism and the cell cycle phase.

10 Enhancers: enhancers are cis-acting elements that can regulate transcription from nearby genes and function by acting as binding sites for transcription factors.

Gene: A gene as understood herein refers to a gene or a putative gene. The gene may code for a selection marker, a protein of interest, a peptide, a secondary metabolite, or 15 it may be a gene resulting in the production of a miRNA, a siRNA, a tRNA, or any gene which can be transcribed and/or translated.

Guiding means: in the present context, the term refers to an element capable of guiding a nuclease such as Cas 9 towards its target. Guiding means can be for example a 20 single guide RNA (sgRNA) or a crRNA/tracrRNA set.

Homologous Recombination (HR): Homologous Recombination is one of the two major pathways for repairing DSBs. HR is a type of genetic recombination in which nucleotide sequences are exchanged between two similar or identical molecules of DNA. HR involves copying information from a donor DNA. The terms HR and HDR (homologydirected repair) are herein used interchangeably.

Homology arm or homologous recombination (HR) template: the term covers a stretch of DNA with sequences homologous to the upstream and downstream regions of a region of interest, in particular of a cut site or a targeted endonuclease site.

Indel: an indel refers to a mutation class, resulting in an insertion and/or a deletion of nucleotides, leading to a net change in the total number of nucleotides. The change in the total number of nucleotides is typically in the range of 1 to 5 nucleotides, but may be up to 100 nucleotides or more. 
Knockdown: the term refers to the process by which genes transcription levels can be reduced in an organism.

5

Knockin: the term refers to the process by which genes can be inserted in a genome. The inserted genes may be genes from the same organism or from other species.

Knockout: the term refers to the process by which genes can be inactivated in an organism, for example by deletion or mutation of part or all of the gene, or of part or all of the elements necessary for the gene to be expressed in a functional protein.

Multiplex editing: the term refers herein to editing nucleic acid sequences of multiple sequences, which can be performed simultaneously or serially. For example, multiplex editing may refer to serial knockins and/or serial knockouts or a combination of knockins and knockouts. It may also refer to simultaneous knockins and/or knockouts of multiple target nucleic acid sequences.

Nick: a nick is a discontinuity in a double-stranded DNA molecule where there is no 20 phosphodiester bond between adjacent nucleotides of one strand.

Non-Homologous End Joining (NHEJ): NHEJ is one of the two major pathways for repairing DSBs. The NHEJ pathway harbours four NHEJ activities defined below, which usually involve at least one Ku protein and a ligase. The two ends at the break are joined directly. The ends at the break may be resected prior to repair, which may lead to loss of some nucleotides and improper repair. Thus NHEJ is often error-prone.

NHEJ activity: the term 'activity' as used herein may refer to a protein activity such as an enzymatic activity involved in the NHEJ pathway. In particular, the term is used to refer to a domain, a peptide or a protein capable of acting as a ligase, or as a polymerase, or as a primase, or as a protein capable of binding DNA ends around a break. The DNA binding activity is typically performed by one or more Ku proteins. The ligase and primase activities can be performed by a single protein, such as ligase $D$. Ligase $D$ can however also be capable of performing only one of the primase or ligase or polymerase activities. A fully functional NHEJ pathway comprises all four activities, 
while a partly functional or partly deficient NHEJ lacks at least one of these four activities.

Nuclear Localisation Sequence (NLS): a nuclear localisation signal or sequence (NLS) is an amino acid sequence which 'tags' a protein for import into the cell nucleus by nuclear transport. Typically, this signal consists of one or more short sequences of positively charged lysines or arginines exposed on the protein surface. Different nuclear localised proteins may share the same NLS. An NLS has the opposite function of a nuclear export signal, which targets proteins out of the nucleus.

10

Nucleic acid: the term refers herein to a sequence of nucleotides.

Parasiticide: the term is to be understood in its broadest sense as an agent capable of inactivating or killing any undesirable organism and thus comprises insecticides, anthelmintic compounds, larvacides, antiparasitic agents and antiprotozoal agents.

Polynucleotide / Oligonucleotide: the terms "polynucleotide" and "oligonucleotide" as used herein denote a nucleic acid chain. Throughout this application, nucleic acids are designated starting from the 5 '-end.

20

Promoter: a promoter is a DNA sequence near the beginning of a gene (typically upstream) that signals the RNA polymerase where to initiate transcription. Eukaryotic promoters may comprise regulatory elements several kilobases upstream of the gene and typically bind transcription factors involved in the formation of the transcriptional 25 complex. Promoters may be inducible, i.e. their activity may be induced by the presence or absence of a biotic or abiotic compound.

Recognition: as understood herein, the term 'recognition' refers to the ability of a molecule to identify a nucleotide sequence. Certain enzymes may require the presence of additional recognition means, such as guiding RNAs or DNA binding domains, to efficiently recognise their substrate sequence. For example, an enzyme or a DNA binding domain may recognise a nucleic acid sequence as a potential substrate and bind to it. Guiding means such as sgRNAs or crRNA/tracrRNA sets may recognise a specific sequence to which they are at least partly homologous. 
Recombinase: as understood herein, the term 'recombinase' refers to an enzyme that can catalyse directionally sensitive DNA exchange reactions between short (30-40 nucleotides) target site sequences. These reactions enable four basic functional modules, excision/insertion, inversion, translocation and cassette exchange.

Terminator: a terminator is a DNA sequence near the end of a gene (typically downstream) that signals the RNA polymerase where to stop transcription. Eukaryotic terminators are recognized by protein factors and termination is followed by polyadenylation of the mRNA.

10

\section{CRISPR-Cas9 system}

The invention relates to methods for gene editing around or modulation of the transcription of at least one target nucleic acid sequence in a host cell based on the use of a CRISPR-Cas9 system. The terms 'target nucleic acid sequence' and 'target sequence' will be used interchangeably.

It will be understood that throughout this document, the term 'CRISPR-Cas9' system refers to a system comprising a CRISPR-Cas9 protein and at least one guiding means, so that the CRISPR-Cas9 system is capable of recognising at least one target nucleic

20 acid sequence. In some embodiments, the CRISPR-Cas 9 system is capable of generating a break in the target nucleic acid sequence, such as a nick on one of the two strands or a double-strand break. Thus the CRISPR-Cas9 system herein comprises Cas 9 and at least one guiding means, where the guiding means is capable of directing Cas9 to its target nucleic acid sequence. The guiding means may be any guiding means known in the art and suitable for this purpose. In some embodiments, the guiding means is a single guide RNA. In other embodiments, the guiding means is a set of a crRNA and a tracrRNA. The skilled person knows how to design guiding means which direct the CRISPR-Cas9 system to a desired target nucleic acid sequence.

30 The nucleic acid sequence encoding Cas9 may be present in the genome of the host cell, e.g. on a chromosome of the host cell, or it may be present on a vector comprised within the host cell. Likewise, the guiding means may be present in the genome of the host cell, e.g. on a chromosome of the host cell, or it may be present on a vector comprised within the host cell. The term 'present in the genome of the host cell' means that either the Cas9 gene or the guiding means are naturally present in the genome of the 
host cell or that they has been introduced e.g. by genome editing and conventional transformation.

In embodiments where the nucleic acid sequence encoding Cas9 and the guiding means are comprised within a vector, Cas 9 and the guiding means may be comprised within the same vector. In embodiments where the guiding means are comprised within a vector and the guiding means is a crRNA and a tracrRNA, the nucleic acid sequences for the crRNA and the tracrRNA may be comprised within two different vectors. The nucleic acid sequence encoding Cas 9 may then be comprised within one of these two vectors, within a third vector or within the genome of the host cell.

The CRISPR-Cas9 system used for the methods disclosed herein may be capable of generating a break in at least one target nucleic acid sequence, such as in at least two target nucleic acid sequences, such as in at least three target nucleic acid sequences, 15 such as in at least four target nucleic acid sequences, such as in at least five target nucleic acid sequences. The CRISPR-Cas9 system can thus be used for multiplex editing.

The skilled person knows how to adapt the CRISPR-Cas9 system recognising more 20 than one target nucleic acid sequence. By way of illustration, the system may comprise two different sgRNAs that each target one target nucleic acid sequence when recognition of two target nucleic acid sequences is desired, or the system may comprise one sgRNA targeting a first target nucleic acid sequence and a crRNA and tracrRNA targeting a second target nucleic acid sequence. Where editing of three target sequences is 25 desired, three different sgRNAs can be used, or two different sgRNAs each targeting a first and a second target sequence and a crRNA and tracrRNA targeting a third sequence, or one sgRNA targeting a first sequence and two sets of crRNA and tracrRNA each targeting a second and a third sequence, or three sets of crRNA and tracrRNA each targeting a different target sequence.

The sequences of the nucleic acid(s) encoding the elements of the CRISPR-Cas9 system may be codon-optimized depending on the host cell in which gene editing is to be performed. Methods for codon optimization are known in the art. 
Host cell

The methods of the present invention allow editing of at least one target nucleic acid sequence comprised within a host cell.

5 The present method can be performed in an archaea, in a prokaryotic cell or in a eukaryotic cell. In one embodiment, the host cell is a prokaryotic cell. The present methods are particularly advantageous for gene editing in host cells that have a high GC content and where gene editing can be difficult to perform. In some embodiments, the GC content is higher than $50 \%$ or more, such as $55 \%$ or more, such as $60 \%$ or more, such as $65 \%$ or more, such as $70 \%$ or more, such as $75 \%$ or more, such as $80 \%$ or more. In a particular embodiment, the host cell is an actinobacterium. The host cell may be selected from the group consisting of Actinomycetales, such as Streptomyces sp., Amycolatopsis sp. or Saccharopolyspora sp. In some embodiments, the host cell is selected from the group consisting of Streptomyces coelicolor, Streptomyces avermiti-

15 lis, Streptomyces aureofaciens, Streptomyces griseus, Streptomyces parvulus, Streptomyces albus, Streptomyces vinaceus, Streptomyces acrimycinis, Streptomyces calvuligerus, Streptomyces lividans, Streptomyces limosus, Streptomyces rubiqinosis, Streptomyces azureus, Streptomyces glaucenscens, Streptomyces rimosus, Streptomyces violaceoruber, Streptomyces kanamyceticus, Amycolatopsis orientalis, Amyco-

20 latopsis mediterranei and Saccharopolyspora erythraea. In a preferred embodiment, the host cell is Streptomyces coelicolor.

In some embodiments, the host cell is from the order Micromonosporales, in particular from the family Micromonosporaceae. In one embodiment, the genus of the host cell is selected from Actinocatenispora, Actinoplanes, Allocatelliglobosispora, Asanoa, Catellatospora, Catelliglobosispora, Catenuloplanes, Couchioplanes, Dactylosporangium, Hamadaea, Jishengella, Krasilnikovia, Longispora, Luedemannella, Micromonospora, Phytohabitans, Phytomonospora, Pilimelia, Planosporangium, Plantactinospora, Polymorphospora, Pseudosporangium, Rhizocola, Rugosimonospora, Salinispora, Solwaraspora, Spirilliplanes, Verrucosispora, Virgisporangium, Wangella or Xiangella.

In some embodiments, the host cell is from the order Streptomycetales, in particular from the family Streptomycetaceae. In one embodiment, the genus of the host cell is selected from Kitasatospora, Parastreptomyces, Streptacidiphilus, Streptomyces or Trichotomospora. 
In some embodiments, the host cell is from the order Propionibacteriales, in particular from the family Nocardioidaceae. In one embodiment, the genus of the host cell is selected from Actinopolymorpha, Aeromicrobium, Flindersiella, Friedmanniella, Kribbella, Marmoricola, Micropruina, Mumia, Nocardioides, Pimelobacter, Propionicicella, Propionicimonas, Tenggerimyces or Thermasporomyces.

In some embodiments, the host cell is from the order Propionibacteriales, in particular from the family Propionibacteriaceae. In one embodiment, the genus of the host cell is selected from Aestuariimicrobium, Auraticoccus, Brooklawnia, Granulicoccus, Luteococcus, Mariniluteicoccus, Microlunatus, Naumannella, Ponticoccus, Propionibacterium, Propioniciclava, Propioniferax, Propionimicrobium or Tessaracoccus.

In some embodiments, the host cell is from the order Pseudonocardiales, in particular from the family Pseudonocardiaceae. In one embodiment, the genus of the host cell is selected from Actinoalloteichus, Actinokineospora, Actinomycetospora, Actinophytocola, Actinorectispora, Actinosynnema, Alloactinosynnema, Allokutzneria, Amycolatopsis, Crossiella, Goodfellowiella, Haloechinothrix, Kibdelosporangium, Kutzneria, Labedaea, Lechevalieria, Lentzea, Longimycelium, Prauserella, Prauseria, Pseudonocardia, Saccharomonospora, Saccharopolyspora, Saccharothrix, Saccharothrixopsis,

20 Sciscionella, Streptoalloteichus, Tamaricihabitans, Thermocrispum, Thermotunica, Umezawaea or Yuhushiella.

In some embodiments, the host cell is from the order Streptosporangiales, in particular from the family Nocardiopsaceae. In one embodiment, the genus of the host cell is selected from Allosalinactinospora, Haloactinospora, Marinactinospora, Murinocardiopsis, Nocardiopsis, Salinactinospora, Spinactinospora, Streptomonospora or Thermobifida.

In some embodiments, the host cell is from the order Streptosporangiales, in particular from the family Streptosporangiaceae. In one embodiment, the genus of the host cell is selected from Acrocarpospora, Astrosporangium, Clavisporangium, Herbidospora, Microbispora, Microtetraspora, Nonomuraea, Planobispora, Planomonospora, Planotetraspora, Sinosporangium, Sphaerimonospora, Sphaerisporangium, Streptosporangium, Thermoactinospora, Thermocatellispora or Thermopolyspora. 
In some embodiments, the host cell is from the order Streptosporangiales, in particular from the family Thermomonosporaceae. In one embodiment, the genus of the host cell is selected from Actinoallomurus, Actinocorallia, Actinomadura, Spirillospora or Thermomonospora.

5

The following table lists examples of species for the host cell.

Table 1. Non-exhaustive list of suitable host cells.

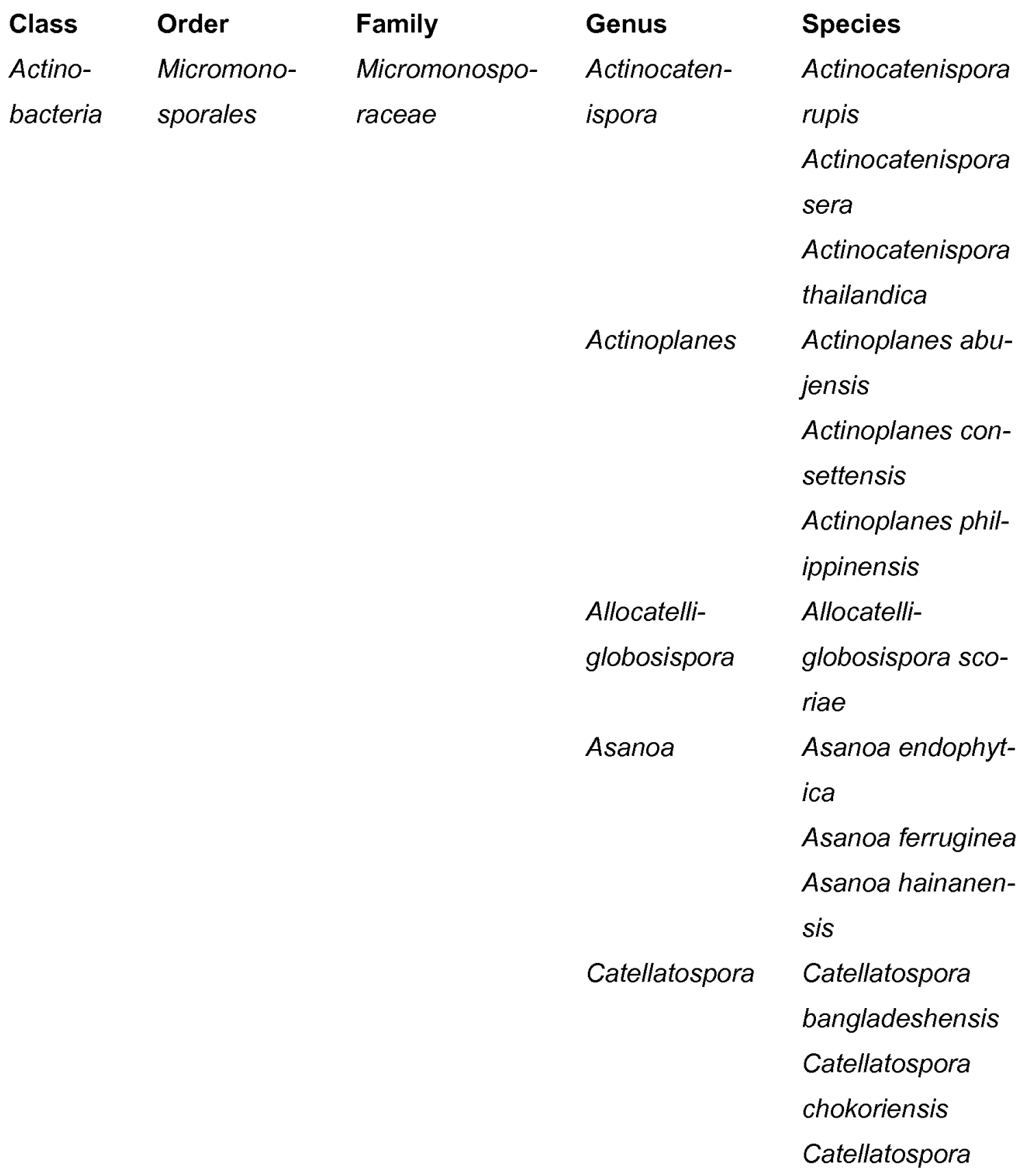




\begin{tabular}{|c|c|}
\hline & citrea \\
\hline Catelli- & Catelliglobosispo- \\
\hline globosispora & ra koreensis \\
\hline \multirow[t]{6}{*}{ Catenuloplanes } & Catenuloplanes \\
\hline & atrovinosus \\
\hline & Catenuloplanes \\
\hline & castaneus \\
\hline & Catenuloplanes \\
\hline & crispus \\
\hline \multirow[t]{2}{*}{ Couchioplanes } & Couchioplanes \\
\hline & caeruleus \\
\hline \multirow{6}{*}{$\begin{array}{l}\text { Dactylosporan- } \\
\text { gium }\end{array}$} & Dactylosporangi- \\
\hline & um darangshiense \\
\hline & Dactylosporangi- \\
\hline & um fulvum \\
\hline & Dactylosporangi- \\
\hline & um luridum \\
\hline \multirow[t]{3}{*}{ Hamadaea } & Hamadaea flava \\
\hline & Hamadaea tsuno- \\
\hline & ensis \\
\hline \multirow[t]{2}{*}{ Jishengella } & Jishengella endo- \\
\hline & phytica \\
\hline \multirow[t]{2}{*}{ Krasilnikovia } & Krasilnikovia cin- \\
\hline & namomea \\
\hline \multirow[t]{2}{*}{ Longispora } & Longispora albida \\
\hline & Longispora fulva \\
\hline \multirow[t]{4}{*}{ Luedemannella } & Luedemannella \\
\hline & flava \\
\hline & Luedemannella \\
\hline & helvata \\
\hline \multirow{5}{*}{$\begin{array}{l}\text { Micromonospo- } \\
\text { ra }\end{array}$} & Micromonospora \\
\hline & aquatica \\
\hline & Micromonospora \\
\hline & arenae \\
\hline & Micromc \\
\hline
\end{tabular}


arenincolae

Phytohabitans Phytohabitans

flavus

Phytohabitans

houttuyneae

Phytohabitans

rumicis

Phytomonospo-

Phytomonospora

ra

endophytica

Pilimelia

Pilimelia anulata

Pilimelia columellifera

Planosporangi- Planosporangium um flavigriseum

Planosporangium mesophilum

Planosporangium thailandense

Plantactinospo- Plantactinospora

ra endophytica Plantactinospora mayteni

Plantactinospora siamensis

Polymor- Polymorphospora phospora rubra

Pseudosporan- Pseudosporangigium um ferrugineum

Rhizocola Rhizocola hellebori

Rugosimono- Rugosimonospora spora acidiphila Rugosimonospora africana

Salinispora alinispora arenico- 
la

Salinispora pacifi-

ca

Salinispora tropica

Solwaraspora

Spirilliplanes

Spirilliplanes ya-

manashiensis

Verrucosispora Verrucosispora

andamanensis

Verrucosispora

fiedleri

Verrucosispora

gifhornensis

Virgisporangium Virgisporangium

aliadipatigenens

Virgisporangium

aurantiacum

Virgisporangium

ochraceum

Wangella Wangella har-

binensis

Xiangella Xiangella phaseoli

Streptomy- Streptomyceta- Kitasatospora

Kitasatospora ar-

boriphila

Kitasatospora vi-

ridis

Kitasatospora

cystarginea

Parastreptomy- Parastreptomyces

ces abscessus

Streptacidiphi- Streptacidiphilus lus albus

Streptacidiphilus

griseus

Streptacidiphilus 


\begin{tabular}{|c|c|}
\hline & rugosus \\
\hline & Streptacidiphilus \\
\hline & thailandensis \\
\hline & Streptacidiphilus \\
\hline & carbonis \\
\hline Streptomyces & Streptomyces al- \\
\hline & bidoflavus group \\
\hline & Streptomyces ac- \\
\hline & rimycinis \\
\hline & Streptomyces \\
\hline & avermitilis \\
\hline & Streptomyces au- \\
\hline & reofaciens \\
\hline & Streptomyces al- \\
\hline & bus \\
\hline & Streptomyces az- \\
\hline & ureus \\
\hline & Streptomyces catt- \\
\hline & leya \\
\hline & Streptomyces \\
\hline & clavuligerus \\
\hline & Streptomyces col- \\
\hline & linus \\
\hline & Streptomyces eu- \\
\hline & rocidicus \\
\hline & Streptomyces \\
\hline & erythrogriseus \\
\hline & Streptomyces fil- \\
\hline & amentosus \\
\hline & Streptomyces \\
\hline & fradiae \\
\hline & Streptomyces \\
\hline & griseus group \\
\hline & Streptomyces \\
\hline & glaucenscens \\
\hline
\end{tabular}


Streptomyces himastatinicus

Streptomyces hygroscopicus Streptomyces hygrospinosus Streptomyces kanamyceticus Streptomyces lactacystinaeus Streptomyces lavendulae Streptomyces levis Streptomyces libani

Streptomyces limosus Streptomyces lividans Streptomyces lomondensis Streptomyces marinus

Streptomyces melanosporofaciens group Streptomyces mexicanus Streptomyces mobaraensis Streptomyces polyantibioticus Streptomyces parvulus

Streptomyces pur- 


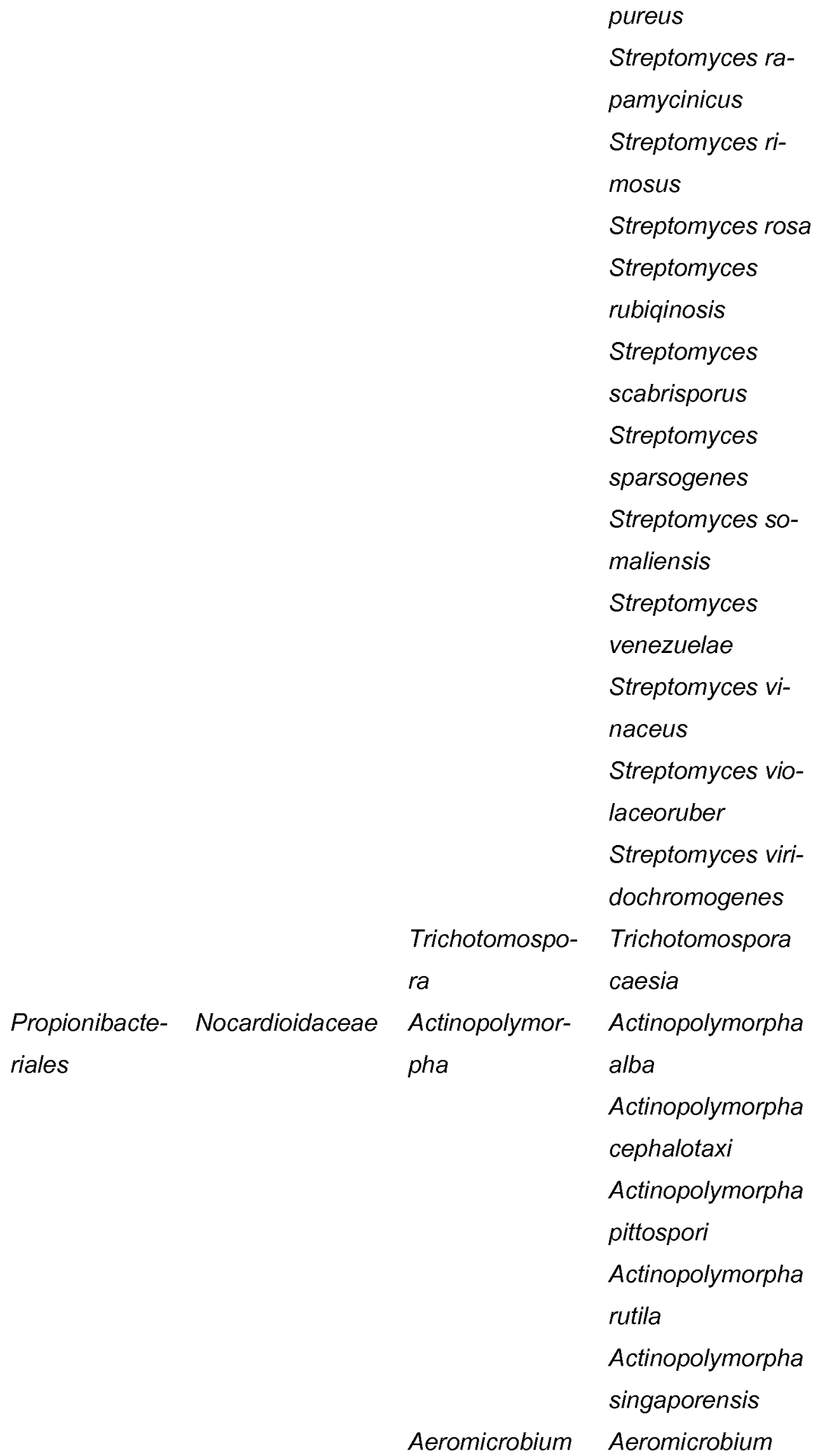


fastidiosum

Aeromicrobium

flavum

Aeromicrobium

ginsengisoli

Aeromicrobium

halocynthiae

Aeromicrobium

kazakhstani

Aeromicrobium

kwangyangensis

Aeromicrobium

marinum

Flindersiella

Flindersiella endo-

phytica

Friedmanniella

Friedmanniella

aerolata

Friedmanniella

antarctica

Friedmanniella

capsulata

Friedmanniella

flava

Friedmanniella

lacustris

Friedmanniella

lucida

Friedmanniella

luteola

Friedmanniella

okinawensis

Friedmanniella

sagamiharensis

Friedmanniella

spumicola 


\begin{tabular}{|c|c|}
\hline \multirow[t]{12}{*}{ Kribbella } & Kribbella alba \\
\hline & Kribbella alber- \\
\hline & tanoniae \\
\hline & Kribbella alumino- \\
\hline & sa \\
\hline & Kribbella am- \\
\hline & oyensis \\
\hline & Kribbella antibioti- \\
\hline & ca \\
\hline & Kribbella cata- \\
\hline & cumbae \\
\hline & Kribbella flavida \\
\hline \multirow[t]{17}{*}{ Marmoricola } & Marmoricola ae- \\
\hline & quoreus \\
\hline & Marmoricola \\
\hline & aquaticus \\
\hline & Marmoricola au- \\
\hline & rantiacus \\
\hline & Marmoricola bige- \\
\hline & umensis \\
\hline & Marmoricola gin- \\
\hline & sengisoli \\
\hline & Marmoricola ko- \\
\hline & recus \\
\hline & Marmoricola \\
\hline & pocheonesis \\
\hline & Marmoricola sco- \\
\hline & riae \\
\hline & Marmoricola soli \\
\hline \multirow[t]{2}{*}{ Micropruina } & Micropruina glyco- \\
\hline & genica \\
\hline Mumia & Mumia flava \\
\hline \multirow[t]{3}{*}{ Nocardioides } & Nocardioides aes- \\
\hline & tuarii \\
\hline & Nocardioides \\
\hline
\end{tabular}




\begin{tabular}{|c|c|c|}
\hline & & agariphilus \\
\hline & & Nocardioides al- \\
\hline & & bertanoniae \\
\hline & & Nocardioides albi- \\
\hline & & dus \\
\hline & & Nocardioides al- \\
\hline & & bus \\
\hline & Pimelobacter & $\begin{array}{l}\text { Pimelobacter sim- } \\
\text { plex }\end{array}$ \\
\hline & Propionicicella & Propionicicella \\
\hline & & superfundia \\
\hline & Propionici- & Propionicimonas \\
\hline & monas & paludicola \\
\hline & Tenggerimyces & Tenggerimyces \\
\hline & & flavus \\
\hline & & Tenggerimyces \\
\hline & & mesophilus \\
\hline & Thermasporo- & Thermasporomy- \\
\hline & myces & ces composti \\
\hline Propionibacteri- & Aestuariimicro- & Aestuariimicrobi- \\
\hline aceae & bium & um kwangyangen- \\
\hline & & se \\
\hline & Auraticoccus & Auraticoccus \\
\hline & & monumenti \\
\hline & Brooklawnia & Brooklawnia cer- \\
\hline & & clae \\
\hline & & Brooklawnia mas- \\
\hline & & siliensis \\
\hline & Granulicoccus & Granulicoccus \\
\hline & & phenolivorans \\
\hline & Luteococcus & Granulicoccus \\
\hline & & phenolivorans \\
\hline & & Luteococcus peri- \\
\hline & & tonei \\
\hline & & Luteococcus san- \\
\hline
\end{tabular}




\begin{tabular}{|c|c|}
\hline & guinis \\
\hline & Luteococcus \\
\hline & sediminum \\
\hline Mariniluteicoc- & Mariniluteicoccus \\
\hline cus & endophyticus \\
\hline & Mariniluteicoccus \\
\hline & flavus \\
\hline Microlunatus & $\begin{array}{l}\text { Microlunatus au- } \\
\text { rantiacus }\end{array}$ \\
\hline & $\begin{array}{l}\text { Microlunatus en- } \\
\text { dophyticus }\end{array}$ \\
\hline & Microlunatus gin- \\
\hline & sengisoli \\
\hline & Microlunatus gin- \\
\hline & sengiterrae \\
\hline & Microlunatus \\
\hline & panaciterrae \\
\hline & Microlunatus pari- \\
\hline & etis \\
\hline Naumannella & Naumannella halo- \\
\hline & tolerans \\
\hline Ponticoccus & Ponticoccus gilvus \\
\hline Propionibacte- & Propionibacterium \\
\hline rium & acidifaciens \\
\hline & Propionibacterium \\
\hline & acidipropionici \\
\hline & ropionibacterium \\
\hline & acnes \\
\hline & Propionibacterium \\
\hline & avidum \\
\hline Propioniciclava & Propioniciclava \\
\hline & tarda \\
\hline Propioniferax & Propioniferax in- \\
\hline & nocua \\
\hline Propionimicro- & Propionimicrobium \\
\hline
\end{tabular}




\begin{tabular}{|c|c|c|c|}
\hline & & bium & lymphophilum \\
\hline & & Tessaracoccus & Tessaracoccus \\
\hline & & & bendigoensis \\
\hline & & & Tessaracoccus \\
\hline & & & flavescens \\
\hline & & & Tessaracoccus \\
\hline & & & flavus \\
\hline & & & Tessaracoccus \\
\hline & & & lapidicaptus \\
\hline & & & Tessaracoccus \\
\hline & & & lubricantis \\
\hline & & & Tessaracoccus \\
\hline & & & oleiagri \\
\hline & & & Tessaracoccus \\
\hline & & & profundi \\
\hline & & & Tessaracoccus \\
\hline & & & rhinocerotis \\
\hline Pseudonocar- & Pseudonocardi- & Actinoalloteich- & Actinoalloteichus \\
\hline diales & aceae & us & alkalophilus \\
\hline & & & Actinoalloteichus \\
\hline & & & cyanogriseus \\
\hline & & Actinokineospo- & Actinokineospora \\
\hline & & ra & auranticolor \\
\hline & & & Actinokineospora \\
\hline & & & baliensis \\
\hline & & & Actinokineospora \\
\hline & & & bangkokensis \\
\hline & & & Actinokineospora \\
\hline & & & cianjurensis \\
\hline & & & Actinokineospora \\
\hline & & & cibodasensis \\
\hline & & & Actinokineospora \\
\hline & & & diospyrosa \\
\hline & & & Actinokineospora \\
\hline & & & enzanensis \\
\hline
\end{tabular}


Actinokineospora inagensis

Actinomyce- Actinomycetospotospora ra chiangmaiensis Actinomycetospora chibensis Actinomycetospora chlora Actinomycetospora cinnamomea

Actinophytocola Actinophytocola burenkhanensis Actinophytocola corallina

Actinophytocola gilvus

Actinophytocola oryzae Actinophytocola sediminis Actinophytocola timorensis Actinophytocola xinjiangensis

Actinorectispora

Actinorectispora indica

Actinosynnema Actinosynnema mirum

AlloactiAlloactinosynnema nosynnema album Alloactinosynnema iranicum

Allokutzneria Allokutzneria albata

Allokutzneria mul- 


\begin{tabular}{|c|c|}
\hline & tivorans \\
\hline & Allokutzneria ory- \\
\hline & zae \\
\hline Amycolatopsis & Amycolatopsis \\
\hline & alba \\
\hline & Amycolatopsis \\
\hline & azurea \\
\hline & Amycolatopsis \\
\hline & coloradensis \\
\hline & Amycolatopsis \\
\hline & coloradensis \\
\hline & Amycolatopsis \\
\hline & halophila \\
\hline & Amycolatopsis \\
\hline & lurida \\
\hline & Amycolatopsis \\
\hline & mediterranei \\
\hline & Amycolatopsis \\
\hline & pigmentata \\
\hline & Amycolatopsis \\
\hline & taiwanensis \\
\hline Crossiella & Crossiella cryophi- \\
\hline & la \\
\hline & Crossiella equi \\
\hline Goodfellowiella & Goodfellowiella \\
\hline & coeruleoviolacea \\
\hline Haloechinothrix & Haloechinothrix \\
\hline & alba \\
\hline Kibdelosporan- & Haloechinothrix \\
\hline gium & alba \\
\hline Kutzneria & Kutzneria albida \\
\hline Labedaea & Labedaea rhizo- \\
\hline & sphaerae \\
\hline Lechevalieria & Lechevalieria \\
\hline & aerocolonigenes \\
\hline
\end{tabular}


Lechevalieria atacamensis

Lechevalieria deserti

Lechevalieria flava Lechevalieria fradiae

Lechevalieria nigeriaca Lechevalieria roselyniae Lechevalieria xinjiangensis

Lentzea albida

Lentzea albidocapillata

Lentzea californiensis

Lentzea flaviverrucosa

Lentzea jiangxiensis

Lentzea kentuckyensis

Lentzea violacea

Lentzea waywayandensis

Longimycelium Longimycelium tulufanense

Prauserella Prauserella aidingensis Prauserella alba Prauserella coralliicola

Prauserella flava 


$\begin{array}{ll}\text { Prauseria } & \text { Prauseria hordei } \\ \text { Pseudonocardia } & \text { Pseudonocardia } \\ & \text { acaciae } \\ & \text { Pseudonocardia } \\ & \text { asaccharolytica } \\ & \text { Pseudonocardia } \\ \text { spinosispora } & \text { Pseudonocardia } \\ \text { sulfidoxydans }\end{array}$

Pseudonocardia tetrahydrofuranoxydans

Pseudonocardia tetrahydrofuranoxydans

Saccharomono- Saccharomonospora spora azurea Saccharomonospora cyanea Saccharomonospora viridis Saccharomonospora marina Saccharopoly- Saccharopolyspospora ra antimicrobica Saccharopolyspora cavernae Saccharopolyspora cebuensis Saccharopolyspora dendranthemae Saccharopolyspora emeiensis Saccharopolyspora endophytica 
Saccharopolyspo-

ra erythraea

Saccharopolyspora spinosa

Saccharopolyspora rosea

Saccharothrix

Lentzea flavoverrucoides

Saccharothrix algeriensis

Saccharothrix australiensis

Saccharothrix carnea

Saccharothrix coeruleofusca

Saccharothrix espanaensis

Saccharothrix- Saccharothrixopsis opsis albidus

Sciscionella Sciscionella marina

Streptoal- Streptoalloteichus loteichus hindustanus Streptoalloteichus tenebrarius

Tamaricihabit- Tamaricihabitans ans halophyticus

Thermocrispum Thermocrispum agreste Thermocrispum municipale

Thermotunica Thermotunica guangxiensis Umezawaea Umezawaea tan- 


\begin{tabular}{|c|c|c|c|}
\hline & & & gerina \\
\hline & & Yuhushiella & Yuhushiella deser- \\
\hline & & & $t i$ \\
\hline Streptospo- & Nocardiopsaceae & Allosalinactino- & Allosalinactinospo- \\
\hline rangiales & & spora & ra lopnorensis \\
\hline & & Haloactinospora & Haloactinospora \\
\hline & & & alba \\
\hline & & Marinactinospo- & Marinactinospora \\
\hline & & ra & thermotolerans \\
\hline & & Murinocardi- & Murinocardiopsis \\
\hline & & opsis & flavida \\
\hline & & Nocardiopsis & Nocardiopsis ae- \\
\hline & & & gyptia \\
\hline & & & Nocardiopsis alba \\
\hline & & & Nocardiopsis alge- \\
\hline & & & riensis \\
\hline & & & Nocardiopsis al- \\
\hline & & & kaliphila \\
\hline & & & Nocardiopsis \\
\hline & & & baichengensis \\
\hline & & & Nocardiopsis \\
\hline & & & chromatogenes \\
\hline & & & Nocardiopsis gan- \\
\hline & & & jiahuensis \\
\hline & & & Nocardiopsis lu- \\
\hline & & & centensis \\
\hline & & & Nocardiopsis po- \\
\hline & & & tens \\
\hline & & & Nocardiopsis \\
\hline & & & synnema- \\
\hline & & & taformans \\
\hline & & & Nocardiopsis \\
\hline & & & prasina \\
\hline & & & Nocardiopsis hal- \\
\hline & & & ophila \\
\hline
\end{tabular}


Salinactinospo- Salinactinospora

ra

qingdaonensis

Salinactinospora

qingdaonensis

Spinactinospora Streptomonospora alba

Streptomono- Streptomonospora

spora

algeriensis

Streptomonospora

amylolytica

Streptomonospora

arabica

Streptomonospora

flavalba

Streptomonospora

halophila

Streptomonospora

nanhaiensis

Streptomonospora

salina

Streptomonospora

sediminis

Thermobifida Thermobifida cel-

lulosilytica

Thermobifida fus-

ca

Thermobifida alba

Streptosporan-

Acrocarpospora

Acrocarpospora

giaceae

corrugata

Acrocarpospora

macrocephala

Acrocarpospora

phusangensis

Acrocarpospora

pleiomorpha 


\begin{tabular}{|c|c|}
\hline $\begin{array}{l}\text { Astrosporangi- } \\
\text { um }\end{array}$ & $\begin{array}{l}\text { Astrosporangium } \\
\text { hypotensionis }\end{array}$ \\
\hline $\begin{array}{l}\text { Clavisporangi- } \\
\text { um }\end{array}$ & $\begin{array}{l}\text { Clavisporangium } \\
\text { rectum }\end{array}$ \\
\hline Herbidospora & $\begin{array}{l}\text { Herbidospora cre- } \\
\text { tacea } \\
\text { Herbidospora da- } \\
\text { liensis } \\
\text { Herbidospora } \\
\text { mongoliensis } \\
\text { Herbidospora sa- } \\
\text { kaeratensis } \\
\text { Herbidospora yil- } \\
\text { anensis }\end{array}$ \\
\hline Microbispora & $\begin{array}{l}\text { Microbispora ame- } \\
\text { thystogenes } \\
\text { Microbispora bry- } \\
\text { ophytorum } \\
\text { Microbispora } \\
\text { camponoti } \\
\text { Microbispora cor- } \\
\text { allina } \\
\text { Microbispora gris- } \\
\text { eoalba } \\
\text { Microbispora hai- } \\
\text { nanensis } \\
\text { Microbispora } \\
\text { mesophila } \\
\text { Microbispora } \\
\text { rosea }\end{array}$ \\
\hline Microtetraspora & $\begin{array}{l}\text { Microtetraspora } \\
\text { fusca } \\
\text { Microtetraspora } \\
\text { glauca } \\
\text { Microtetraspora }\end{array}$ \\
\hline
\end{tabular}




\begin{tabular}{|c|c|}
\hline & malaysiensis \\
\hline & Microtetraspora \\
\hline & niveoalba \\
\hline Nonomuraea & Nonomuraea ae- \\
\hline & gyptia \\
\hline & Nonomuraea afri- \\
\hline & cana \\
\hline & Nonomuraea an- \\
\hline & giospora \\
\hline & Nonomuraea an- \\
\hline & timicrobica \\
\hline & Nonomuraea asi- \\
\hline & atica \\
\hline & Nonomuraea au- \\
\hline & rea \\
\hline & Nonomuraea \\
\hline & bangladeshensis \\
\hline & Nonomuraea can- \\
\hline & dida \\
\hline Planobispora & Planobispora long- \\
\hline & ispora \\
\hline & Planobispora \\
\hline & rosea \\
\hline & Planobispora sia- \\
\hline & mensis \\
\hline & Planobispora tak- \\
\hline & ensis \\
\hline Planomonospo- & Planomonospora \\
\hline ra & alba \\
\hline & Planomonospora \\
\hline & parontospora \\
\hline Planotetraspora & Planotetraspora \\
\hline & kaengkrachanen- \\
\hline & sis \\
\hline & Planotetraspora \\
\hline
\end{tabular}


mira

Planotetraspora

phitsanulokensis

Planotetraspora

silvatica

Planotetraspora

thailandica

Sinosporangium Sinosporangium

album

Sinosporangium

siamense

Sphaerimono- Sphaerimonospo-

spora

ra cavernae

Sphaerisporan-

Sphaerisporangi-

gium

um album

Sphaerisporangi-

um cinnabarinum

Sphaerisporangi-

um flaviroseum

Streptosporan- Sphaerisporangigium

um album

Sphaerisporangium cinnabarinum

Sphaerisporangium flaviroseum

Sphaerisporangium krabiense

Sphaerisporangium melleum

Sphaerisporangium rubeum

Sphaerisporangium rufum

Sphaerisporangium siamense 
Sphaerisporangi-

um viridialbum

Thermoactino- Thermoactinospo-

spora ra rubra

Thermoca- Thermocatellispo-

tellispora ra tengchongensis

Thermopolyspo-

ra flexuosa

ThermomonoActinoallomurus

Actinoallomurus sporaceae

caesius
Actinoallomurus
Actinoallomurus
fulvus
Actinoallomurus
iriomotensis
Actinoallomurus
acaciae
Actinoallomurus
acanthiterrae
Actinoallomurus
amamiensis
Actinoallomurus
bryophytorum
Actinocorallia au-
rantiaca
Actinocorallia au-
rea
Actinocorallia
cavernae
Actinocorallia
glomerata
Actinocorallia her-
bida
Actinocorallia liba-
Actinorallia




\begin{tabular}{|c|c|}
\hline & notica \\
\hline & $\begin{array}{l}\text { Actinocorallia lon- } \\
\text { gicatena }\end{array}$ \\
\hline & Actinocorallia \\
\hline & spatholoba \\
\hline Actinomadura & Actinomadura alba \\
\hline & Actinomadura am- \\
\hline & ylolytica \\
\hline & Actinomadura apis \\
\hline & Actinomadura at- \\
\hline & ramentaria \\
\hline & Actinomadura \\
\hline & bangladeshensis \\
\hline & Actinomadura ca- \\
\hline & tellatispora \\
\hline & Actinomadura cel- \\
\hline & lulosilytica \\
\hline & Actinomadura \\
\hline & chibensis \\
\hline Spirillospora & Spirillospora al- \\
\hline & bida \\
\hline & Spirillospora rubra \\
\hline Thermomono- & Thermomonospo- \\
\hline spora & ra curvata \\
\hline & Thermomonospo- \\
\hline & ra chromogena \\
\hline
\end{tabular}

Method for generating random-sized deletions or indels around a target site

In a first aspect, the invention relates to a method for generating at least one deletion

5 around at least one target nucleic acid sequence comprised within a host cell having a non-homologous end-joining (NHEJ) pathway which is at least partly deficient, said method comprising the steps of:

(i) optionally, restoring the full functionality of the NHEJ pathway, 
(ii) inducing a CRISPR-Cas9 system in said host cell, wherein said CRISPR-Cas9 system is able to generate at least one break in said at least one target nucleic acid sequence and wherein the CRISPRCas9 system comprises a Cas9 nuclease and at least one guiding means,

thereby generating:

a. if the method does not comprise step (i), at least one random-sized deletion around said at least one target nucleic acid sequence, wherein said at least one deletion is a random-sized deletion of at least $1 \mathrm{bp}$; or

b. if the method does comprise step (i), at least one indel around said at least one target nucleic acid sequence, wherein said at least one indel is a deletion or insertion of at least1 bp.

15 The methods the present disclosure thus take advantage of the fact that in host cells, wherein the NHEJ pathway is at least partly deficient, a CRISPR-Cas9 system can be induced and generates either random-sized deletions around a target site, or indels around a target site if the functionality of the NHEJ pathway is restored prior to or simultaneously with induction of the CRISPR-Cas9 system.

\section{Method for generating random-sized deletions around a target site}

In some embodiments, the method does not comprise step (i). In other words, the NHEJ pathway is maintained partly deficient. The present disclosure thus provides a method for generating at least one random-sized deletion around at least one target nucleic acid sequence comprised within a host cell having a non-homologous endjoining (NHEJ) pathway which is at least partly deficient, said method comprising the step of inducing a CRISPR-Cas9 system in a host cell, said CRISPR-Cas9 system being able to generate at least one break in said at least one target nucleic acid sequence, thereby generating at least one deletion around said at least one target nucleic acid sequence, wherein said at least one deletion is a deletion of at least $1 \mathrm{bp}$.

The method is based on the surprising finding that performing CRISPR-Cas9 directed gene editing in organisms having a partly deficient NHEJ pathway leads to the generation of random-sized deletions around a target nucleic acid sequence. This is surprising because performing CRISPR-Cas9 directed editing in organisms lacking NHEJ was 
believed to be lethal (Citorik, R. J. et, al 2014, Gomaa, A. et, al 2014, Bikard, D., et, al, 2014). The gene editing is preferably performed without homology arms so that the repair of the at least one break generated by Cas 9 is directed towards the NHEJ pathway. Thus in some embodiments, the method for generating at least one deletion described herein is performed with the proviso that the editing is not done with a homologous template.

In some embodiments, the guiding means comprises at least one sgRNA and/or at least one crRNA/tracrRNA set.

10

Also disclosed herein is a method for generating at least one deletion around at least one target nucleic acid sequence comprised within a host cell having a nonhomologous end-joining (NHEJ) pathway which is at least partly deficient, said method comprising the step of inducing a CRISPR-Cas9 system in a host cell, said CRISPR-Cas9 system being able to generate at least one break in said at least one target nucleic acid sequence, thereby generating at least one deletion around said at least one target nucleic acid sequence, wherein said at least one deletion is a deletion of at least $1 \mathrm{bp}$, wherein the CRISPR-Cas 9 system comprises a Cas 9 nuclease encoded by a polynucleotide having at least $93 \%$ identity with SEQ ID NO: 1 , such as

20 at least $94 \%$ identity, such as at least $95 \%$ identity, such as at least $96 \%$ identity, such as at least $97 \%$ identity, such as at least $98 \%$ identity, such as at least $99 \%$ identity, such as $100 \%$ identity with SEQ ID NO: 1 . In some embodiments, the Cas 9 nuclease is identical to SEQ ID NO: 2.

NHEJ

The method disclosed herein for generating random-sized deletions around at least one target nucleic acid sequence is preferably performed in a host cell wherein the NHEJ pathway is at least partly deficient.

The NHEJ pathway involves four activities dependent on two groups of proteins:

(a) the Ku proteins, which bind to DNA double-strand break ends and are required for the non-homologous end joining;

(b) the ligase, such as the ligase $D$ ligD, which can perform the activities of ligase, polymerase and primase. 
In some embodiments, the NHEJ pathway of the host cell thus lacks at least one of the four NHEJ activities defined as:

- a DNA-binding activity,

- a primase activity,

- a ligase activity,

- a polymerase activity.

The DNA-binding activity is typically performed by Ku proteins such as Ku70, Ku80, or homologues, orthologues or paralogues thereof. The primase activity can be performed by a eukaryotic-archeal DNA primase (EP) or a homologue, an orthologue or a paralogue thereof, or by a ligase $\mathrm{D}$ or a homologue, an orthologue or a paralogue thereof. The ligase activity is typically performed by ligase $D$ or a homologue, an orthologue or a paralogue thereof. The polymerase activity is typically performed by a ligase $D$ or a homologue, an orthologue or a paralogue thereof.

As understood herein, a functional NHEJ pathway comprises all four activities, e.g. it may comprise one Ku protein with a DNA-binding activity and a ligase capable of performing the activities of ligase, polymerase and primase. In some embodiments, the activities of ligase, polymerase and primase are performed by the same or by two, 20 three or four different proteins, peptides or domains. A partly deficient NHEJ pathway lacks at least one of the four activities. In some embodiments, the NHEJ pathway of the host cell thus lacks at least one of the DNA-binding activity, of the ligase activity, of the polymerase activity and of the primase activity. In a preferred embodiment, the NHEJ pathway is partly deficient because the ligase can only perform the primase activity.

25 For example, the Ku proteins are present and functional, but the ligase lacks the ligase activity.

The NHEJ pathway may be deficient because it is naturally deficient in the host cell, or because at least one of the four activities has been inactivated. In some embodiments, the DNA-binding activity is inactivated, e.g. by targeted deletion of the nucleic acid sequence(s) encoding the Ku protein(s). In further embodiments, the primase activity is inactivated. In other embodiments, the ligase activity is inactivated. In yet other embodiments, the polymerase activity is inactivated. Preferably, at least the ligase activity is inactivated. Other methods for inactivating at least one of the four NHEJ activities are known to the skilled person. 
Host cells where the NHEJ pathway is naturally deficient can be identified by methods known in the art, such as gene mining or sequence blasting.

5 The activities referred to above may be performed by a domain, peptide or protein. The nucleic acid sequences encoding the domain, peptide or protein capable of performing said activities may be comprised within the genome of the host cell or may be comprised on a vector.

\section{$10 \quad$ Target nucleic acid}

The method disclosed herein is particularly useful for generating random-sized deletions around at least one target nucleic acid sequence of interest. The present method can thus be used in order to generate clonal libraries containing a plurality of cells having deletions of different sizes around at least one target nucleic acid of interest, as

15 described below. The method can thus be useful for, but not limited to, the investigation of pathway regulations and identification of metabolite production bottlenecks, the screening of producer strains and the identification of new compounds produced by the host cell. The libraries thus generated are not completely random in that the target nucleic acid is predefined.

The target nucleic acid sequence may be comprised within any nucleic acid sequence of interest. For example, the target sequence may be comprised within or may comprise an open reading frame or a putative open reading frame, or it may be comprised within or may comprise a regulatory region or a putative regulatory region, such as an enhancer, a promoter, an insulator, a terminator.

The target nucleic acid sequence may be involved in a pathway of interest. In some embodiments, the target nucleic acid encodes an enzyme or a protein. In other embodiments, the target nucleic acid is comprised within or comprises a biosynthetic gene or a putative biosynthetic gene. In some embodiments, the biosynthetic gene is involved in the synthesis of a secondary metabolite.

In some embodiments, the target nucleic acid sequence is comprised within a gene cluster. In specific embodiments, the gene cluster is a secondary metabolite gene cluster. 
There is thus disclosed herein a method for editing a target nucleic acid sequence optionally comprised within or comprising a gene cluster, where the target nucleic acid sequence is involved or is suspected of being involved in the biosynthesis of a secondary metabolite.

In some embodiments, the secondary metabolite is selected from the group consisting of antibiotics, herbicides, anti-cancer agents, immunosuppressants, flavors, parasiticides and proteins. The term 'parasiticide' is to be understood in its broadest sense as an agent capable of inactivating or killing any undesirable organism and thus comprises insecticides, anthelmintic compounds, larvacides, antiparasitic agents and antiprotozoal agents.

In some embodiments, the secondary metabolite is an antibiotic selected from the group consisting of apramycin, bacitracin, chloramphenicol cephalosporins, cycloserine, erythromycin, fosfomycin, gentamicin, kanamycin, kirromycin, lassomycin, lincomycin, lysolipin, microbisporicin, neomycin, noviobiocin, nystatin, nitrofurantoin, platensimycin, pristinamycins, rifamycin, streptomycin, teicoplanin, tetracycline, tinidazole, ribostamycin, daptomycin, vancomycin, viomycin and virginiamycin.

20

In other embodiments, the secondary metabolite is a herbicide selected from the group consisting of bialaphos, resormycin and phosphinothricin.

In yet other embodiments, the secondary metabolite is an anti-cancer agent selected 25 from the group consisting of doxorubicin, salinosporamides, aclarubicin, pentostatin, peplomycin, thrazarine and neocarcinostatin.

In yet other embodiments, the secondary metabolite is an immunosuppressant selected from the group consisting of rapamycin, FK520, FK506, cyclosporine, ushikulides, pentalenolactone I and hygromycin A.

In yet other embodiments, the secondary metabolite is a flavor such as geosmin. 
In yet other embodiments, the secondary metabolite is a parasiticide such as an insecticide, an anthelmintic, a larvacide, or an antiprotozoal agent such as spinsad or avermectin.

5 In other embodiments, the target nucleic acid codes for an enzyme selected from the group consisting of an amylase, a protease, a cellulase, a chitinase, a keratinase and a xylanase.

In some embodiments, only one target nucleic acid sequence is targeted for editing

10 and generation of random-sized deletions. In other embodiments, more than one target nucleic acid sequence is targeted and the method is a multiplex method. Thus the method can be used for generating at least one deletion around at least one target nucleic acid sequence, such as at least two deletions around at least two target nucleic acid sequences, such as at least three deletions around at least three target nucleic

15 acid sequences, such as at least four deletions around at least four target nucleic acid sequences, such as at least five deletions around at least five target nucleic acid sequences, or more, wherein each deletion as a deletion of at least $1 \mathrm{bp}$. The method can thus be used for generating one deletion around one target nucleic acid sequence, or two deletions around at least two target nucleic acid sequences, or three deletions 20 around three target nucleic acid sequences, or four deletions around four target nucleic acid sequences, or five deletions around five target nucleic acid sequences, or more. As explained above, in the case of multiplex editing, a guiding means is preferably provided for each target nucleic acid sequence.

25 In some embodiments, the at least one deletion results in the inactivation of at least one gene. In some embodiments, the at least one gene is comprised within a gene cluster. In other embodiments, the at least one gene is not comprised within a gene cluster.

30 The at least one deletion generated by the present method is a deletion of at least $1 \mathrm{bp}$ and may range over several thousands kilobases. In some embodiments, the deletion is a deletion of 1 to $2.10^{6} \mathrm{bp}$, such as 1 to $1.10^{6} \mathrm{bp}$, such as 1 to $500000 \mathrm{bp}$, such as 1 to $400000 \mathrm{bp}$, such as 1 to $300000 \mathrm{bp}$, such as 1 to $200000 \mathrm{bp}$, such as 1 to 100000 $\mathrm{bp}$, such as 2 to $75000 \mathrm{bp}$, such as 3 to $50000 \mathrm{bp}$, such as 4 to $40000 \mathrm{bp}$, such as 5 to $30000 \mathrm{bp}$, such as 10 to $20000 \mathrm{bp}$, such as 25 to $10000 \mathrm{bp}$, such as 50 to $9000 \mathrm{bp}$, 
such as 75 to $8000 \mathrm{bp}$, such as 100 to $7000 \mathrm{bp}$, such as 150 to $6000 \mathrm{bp}$, such as 200 to $5000 \mathrm{bp}$, such as 250 to $4000 \mathrm{bp}$, such as 300 to $3000 \mathrm{bp}$, such as 400 to $2000 \mathrm{bp}$, such as 500 to $1000 \mathrm{bp}$, such as 600 to $900 \mathrm{bp}$, such as 700 to $800 \mathrm{bp}$. In some embodiments, the deletion is a deletion of at least $1 \mathrm{bp}$, such as at least $2 \mathrm{bp}$, such as at least $3 \mathrm{bp}$, such as at least $4 \mathrm{bp}$, such as at least $5 \mathrm{bp}$, such as at least $10 \mathrm{bp}$, such as at least $15 \mathrm{bp}$, such as at least $20 \mathrm{bp}$, such as at least $50 \mathrm{bp}$, such as at least $100 \mathrm{bp}$, such as at least $250 \mathrm{bp}$, such as at least $500 \mathrm{bp}$. In some embodiments, the deletion is a deletion of 1 to $100 \mathrm{bp}$, such as 1 to $75 \mathrm{bp}$, such as 1 to $50 \mathrm{bp}$, such as 1 to $40 \mathrm{bp}$, such as 1 to $30 \mathrm{bp}$, such as 1 to $20 \mathrm{bp}$, such as 1 to $10 \mathrm{bp}$, such as 1 to $9 \mathrm{bp}$, such as 1 to $8 \mathrm{bp}$, such as 1 to $7 \mathrm{bp}$, such as 1 to $6 \mathrm{bp}$, such as 1 to $5 \mathrm{bp}$, such as 1 to $4 \mathrm{bp}$, such as 1 to $3 \mathrm{bp}$, such as 1 to $2 \mathrm{bp}$.

\section{Efficiency and off-target effects}

Several parameters can have an impact on the efficiency of the present method for generating random-sized deletions around at least one target sequence. Some parameters can be adjusted as known in the art. Parameters susceptible of having an impact on the efficiency include, but are not limited to: the sequence of the guiding means (sgRNA or crRNA/tracrRNA), the sequence of the target nucleic acid, the GC content of the host cell and the GC content of the target nucleic acid sequence.

The method can be performed with relatively few off-target effects. In some embodiments, the desired deletion is generated in more than $1 \%$ of the host cells, such as in more than $5 \%$ of the host cells, such as in more than $10 \%$ of the host cells, such as in more than $15 \%$ of the host cells, such as in more than $20 \%$ of the host cells, such as in more than $25 \%$ of the host cells, such as in more than $30 \%$ of the host cells, such as in more than $35 \%$ of the host cells, such as in more than $40 \%$ of the host cells, such as in more than $45 \%$ of the host cells, such as in more than $50 \%$ of the host cells, such as in more than $55 \%$ of the host cells, such as in more than $60 \%$ of the host cells, such as in more than $65 \%$ of the host cells, such as in more than $70 \%$ of the host cells, such as in more than $75 \%$ of the host cells, such as in more than $80 \%$ of the host cells, such as in more than $85 \%$ of the host cells, such as in more than $90 \%$ of the host cells, such as in more than $95 \%$ of the host cells, such as in $100 \%$ of the host cells. 


\section{Characterisation and screening}

The present method can thus be used for generating random sized deletions around a target nucleic acid sequence of interest, for example a sequence encoding for a gene involved in a pathway of interest. This can result in a plurality of clones having randomsized deletions around the target sequence. These clones can then be further analysed or screened. For example, producer strains having advantageous production profiles for a desired compound can be selected.

In some embodiments, it may be of interest to determine the size of the at least one deletion for a particular clone. Thus the method may comprise a further step of determining the size of the at least one deletion. Methods for determining the size of a deletion are known in the art and include, but are not limited to, whole genome sequencing, pulsed field gel electrophoresis, nucleic acid amplification-based methods such as PCR, for example followed by restriction analysis and detection of the PCR products on a gel and determination of the size of the products using an appropriate marker. The PCR products can also be sequenced if precise determination of the size of the deletion is desired.

In some embodiments, the method further comprises a step of selection of clones having the desired characteristics. Such selection methods are known in the art and encompass screening methods, chemical analysis of the related gene products (proteins or metabolites), sequencing of the related gene regions, and/or analysis of the gene expression level.

Clonal library

In one aspect, the disclosure relates to a clonal library obtainable by the method for generating random-sized deletions around at least one target nucleic acid sequence as described herein above. Such clonal libraries comprise a plurality of clones obtained by said method, wherein each clone harbours at least one deletion around at least one target nucleic acid sequence, wherein each of said deletions is a deletion of at least 1 bp.

The clonal libraries may be generated by multiplex methods, wherein more than one deletion is generated around more than one target nucleic acid in each clone. 
The clonal libraries may be libraries of archaea, prokaryotes or eukaryotes. In one embodiment, the clonal library is a prokaryotic clonal library. In some embodiments, the clones of the clonal library have a high GC content. In some embodiments, the GC content is higher than $45 \%$, such as $50 \%$ or more, such as $55 \%$ or more, such as $60 \%$ or more, such as $65 \%$ or more, such as $70 \%$ or more, such as $75 \%$ or more, such as $80 \%$ or more. In a particular embodiment, the clonal library is a library of an actinobacterium, for example selected from the group consisting of Actinomycetales, such as Streptomyces sp., Amycolatopsis sp. or Saccharopolyspora sp. In some embodiments, the clonal library is a library of clones derived from Streptomyces coelicolor, Streptomyces avermitilis, Streptomyces aureofaciens, Streptomyces griseus, Streptomyces parvulus, Streptomyces albus, Streptomyces vinaceus, Streptomyces acrimycinis, Streptomyces calvuligerus, Streptomyces lividans, Streptomyces limosus, Streptomyces rubiqinosis, Streptomyces azureus, Streptomyces glaucenscens, Streptomyces rimosus, Streptomyces violaceoruber, Streptomyces kanamyceticus, Amycolatopsis orientalis, Amycolatopsis mediterranei or Saccharopolyspora erythraea. In a preferred embodiment, the clonal library is a library of Streptomyces coelicolor clones.

\section{Method for generating precise indels around a target site}

In some embodiments, the method comprises the step of restoring full functionality of the at least partly deficient NHEJ pathway in the host cell prior to or simultaneously with the step of inducing a CRISPR-Cas9 system. This results in generation of at least one indel around at least one target nucleic acid sequence comprised within a host cell having a non-homologous end-joining (NHEJ) pathway which is at least partly deficient, said method comprising the steps of (i) restoring the full functionality of the NHEJ pathway in said host cell; (ii) inducing a CRISPR-Cas9 system in said host cell, said CRISPR-Cas9 system being able to generate at least one break in said at least one target nucleic acid sequence, thereby generating at least one indel around said at least one target nucleic acid sequence, wherein said at least one indel is an insertion or a deletion of at least 1 bp such as at least 2 bp, such as at least 3 bp, such as at least 4 $\mathrm{bp}$, such as at least $5 \mathrm{bp}$, such as at least $10 \mathrm{bp}$, such as at least $15 \mathrm{bp}$, such as at least $20 \mathrm{bp}$, such as at least $50 \mathrm{bp}$, such as at least $100 \mathrm{bp}$, such as at least $250 \mathrm{bp}$, such as at least $500 \mathrm{bp}$.

In some embodiments, the guiding means comprises at least one sgRNA and/or at least one crRNA/tracrRNA set. 
In a host cell having a partly deficient NHEJ pathway, CRISPR-Cas9 gene editing results in the generation of random-sized deletions around the target sites, as disclosed in the first aspect of the invention. The deletions can, as described above and as shown in the examples, be very large. While this may be of interest in some cases, it may sometimes be desirable to generate precise deletions or insertions around target sequences instead. The terms 'precise deletion' or 'precise insertion' or 'precise indel' preferably refer herein to to insertions, deletions or indels of which the size can be determined in advance, as opposed to random-sized deletions. These can be short deletions, insertions or indels, i.e. spanning over small areas as detailed below. The second aspect of the invention describes how this can be achieved. In some embodiments, the gene editing is performed without homology arms so that the repair of the at least one break generated by Cas9 is directed towards the NHEJ pathway. In other embodiments, the gene editing is performed with homology arms so that the repair of the at least one break generated by Cas 9 is directed toward the HDR pathway.

There is disclosed herein a method for generating at least one indel around at least one target nucleic acid sequence comprised within a host cell having a nonhomologous end-joining (NHEJ) pathway which is at least partly deficient, said method comprising the steps of (i) restoring the full functionality of the NHEJ pathway in said host cell; (ii) inducing a CRISPR-Cas9 system in said host cell, said CRISPR-Cas9 system being able to generate at least one break in said at least one target nucleic acid sequence, thereby generating at least one indel around said at least one target nucleic acid sequence, wherein said at least one indel is an indel of at least $1 \mathrm{bp}$, wherein the CRISPR-Cas 9 system comprises a Cas 9 nuclease encoded by a polynucleotide having at least $93 \%$ identity with SEQ ID NO: 1 , such as at least $94 \%$ identity, such as at least $95 \%$ identity, such as at least $96 \%$ identity, such as at least $97 \%$ identity, such as at least $98 \%$ identity, such as at least $99 \%$ identity, such as $100 \%$ identity with SEQ ID NO: 1. In some embodiments, the Cas9 nuclease is identical to SEQ ID NO: 2.

\section{Restoring NHEJ}

The method disclosed herein for generating precise indels around at least one target nucleic acid sequence is preferably performed in a host cell wherein the NHEJ pathway is at least partly deficient. 
Host cells where the NHEJ pathway is naturally deficient can be identified by methods known in the art, such as gene mining or sequence blasting.

5 The NHEJ pathway involves four activities dependent on two groups of proteins:

(a) the Ku proteins, which bind to DNA double-strand break ends and are required for the non-homologous end joining;

(b) the ligase, such as the ligase $D$ ligD, which can perform the activities of ligase, polymerase and primase.

10

In some embodiments, the NHEJ pathway of the host cell thus lacks at least one of four activities defined as:

- a DNA-binding activity,

- a primase activity,

- a ligase activity

- a polymerase activity.

The DNA-binding activity is typically performed by Ku proteins such as Ku70, Ku80, or homologues, orthologues or paralogues thereof. The primase activity can be performed by a eukaryotic-archeal DNA primase (EP) or a homologue, an orthologue or a paralogue thereof, or by a ligase $\mathrm{D}$ or a homologue, an orthologue or a paralogue thereof. The ligase activity is typically performed ligase $\mathrm{D}$ or a homologue, an orthologue or a paralogue thereof. The polymerase activity is typically performed by a ligase $\mathrm{D}$ or a homologue, an orthologue or a paralogue thereof.

As understood herein, a functional NHEJ pathway comprises all four activities, e.g. it comprises one Ku protein with a DNA-binding activity and a ligase capable of performing the activities of ligase and primase. A partly deficient NHEJ pathway lacks at least one of the four activities. In some embodiments, the NHEJ pathway of the host cell 30 thus lacks at least one of the DNA-binding activity, of the polymerase activity, of the ligase activity and of the primase activity. In a preferred embodiment, the NHEJ pathway is partly deficient because the ligase can only perform the primase activity. For example, the Ku proteins are present and functional, but the ligase lacks the ligase activity. 
The NHEJ pathway may be deficient because it is naturally deficient in the host cell, or because at least one of the four activities has been inactivated. In some embodiments, the DNA-binding activity is inactivated, e.g. by targeted deletion of the nucleic acid sequence(s) encoding the Ku protein(s). In further embodiments, the primase activity is inactivated. In other embodiments, the ligase activity is inactivated. In yet other embodiments, the polymerase activity is inactivated. Preferably, at least the ligase activity is inactivated. Other methods for inactivating at least one of the four NHEJ activities are known to the skilled person.

10 The activities referred to above may be performed by a domain, peptide or protein. The nucleic acid sequences encoding the domain, peptide or protein capable of performing said activities may be comprised within the genome of the host cell or may be comprised on a vector.

15 In order to generate precise indels around at least one target nucleic acid sequence, the at least one NEHJ activity which is lacking in the host cell may need to be restored. This can be achieved by introducing a nucleic acid sequence comprising a sequence encoding a domain, a peptide or a protein capable of performing said lacking NHEJ activity into the host cell.

The nucleic acid sequence comprising a sequence such as an open reading frame encoding said domain, peptide or protein capable of performing said lacking activity (hereinafter also referred to as 'the nucleic acid sequence encoding said lacking activity') can be introduced into the host cell's genome, e.g. on a chromosome, or it can be comprised within a vector and the vector can be introduced within the host cell.

The nucleic acid sequence encoding the lacking NHEJ activity can be under the control of an inducible promoter and may comprise other elements besides an open reading frame encoding the activity. For example, the nucleic acid sequence may further comprise a terminator, a sequence encoding a selection marker and/or a sequence encoding a fluorescent protein.

In some embodiments, the nucleic acid sequence encoding the lacking NHEJ activity and the nucleic acid sequence encoding Cas 9 may be comprised within a single nucleic acid, for example they may be on the same vector or they may be integrated at the 
same location in the genome of the host cell. Likewise, the nucleic acid sequence encoding the lacking NHEJ activity and the nucleic acid sequence encoding the guiding means may be comprised within a single nucleic acid, for example they may be on the same vector or they may be integrated at the same location in the genome of the host cell. In some embodiments, the nucleic acid sequence encoding the lacking NHEJ activity, the nucleic acid sequence encoding Cas 9 and the nucleic acid sequence encoding the guiding means are all comprised within a single nucleic acid. Each of these three elements may also be comprised each within one nucleic acid.

10 In some embodiments, the host cell is lacking more than one NHEJ activity. It may lack two NHEJ activities or it may lack three NHEJ activities or four NHEJ activities. In order to restore NHEJ, it may be necessary to restore each of the lacking activities. The nucleic acid sequences encoding each of the lacking activities can be comprised within a single nucleic acid, or they can be comprised within different nucleic acids. The guiding means and Cas 9 may be comprised within the same nucleic acid as one or all of the sequences encoding the lacking activity, or they may be comprised within a different nucleic acid, as above.

In some embodiments, restoration of the lacking NHEJ activity or activities is achieved 20 by introduction of a heterologous gene encoding a domain, protein or peptide capable of performing the lacking activity when it is expressed in the host cell. Suitable heterologous genes can be identified by methods such as blasting a genome database using a nucleic acid sequence encoding the lacking activity as a query. The query sequence is preferably the sequence of a cell naturally possessing the activity lacking in the host cell in which the method is to be performed. Preferably, the query sequence is taken from a cell which is related to the host cell, for example from a cell which is phylogenetically close to the host cell.

In embodiments where the host cell having a partly deficient NHEJ pathway is an actinobacterium, the cell from which the query sequence is derived is preferably also an actinobacterium.

Once a sequence encoding the lacking activity has been identified, the sequence (hereinafter also termed 'heterologous sequence') may be codon-optimised as is 
known in the art, in order to increase the chances that the heterologous sequence is properly expressed after introduction in the host cell.

The below table shows examples of host cells, the NHEJ actity(ies) they lack and

5 where suitable heterologous genes can be found for restoring the NHEJ pathway.

Table 2 - overview of suitable heterologous genes for host cells lacking various NHEJ activities.

Host cell

Streptomyces griseus,

Streptomyces

acidiscabies,

Streptomyces auratus,

Streptomyces

bottropensis,

Streptomyces chartreusis,

Streptomyces

clavuligerus,

Streptomyces

coelicoflavus,

Streptomyces gancidicus,

Streptomyces ghanaensis,

Streptomyces globisporus,

Streptomyces

griseoaurantiacus,

Streptomyces

griseoflavus,

Streptomyces

himastatinicus,

Streptomyces ipomoeae,

Streptomyces lividans,

Streptomyces

mobaraensis,
Lacking activity(ies) Suitable heterologous

genes can be found in

(non-exhaustive list)

Mycobacterium tuberculo-

sis H37Rv, Mycobacte-

rium canettii, Mycobacte-

rium spp., Rhodococcus

erythropolis, Rhodococcus

equi, Rhodococcus fasci-

ans, Rhodococcus rhodo-

chrous, Rhodococcus

spp., Nocardia araoensis,

Nocardia transvalensis,

Nocardia exalbida, Nocar-

dia spp., Tomitella bifor-

mata, Amycolatopsis med-

iterranei, Amycolatopsis

orientalis, Saccharopoly-

spora erythraea, Pseu-

donocardia dioxanivorans,

Ralstonia pickettii, Krib-

bella flavida, Saccharo-

thrix espanaensis, Sino-

rhizobium meliloti, Actino-

planes friuliensis, Steno-

trophomonas maltophilia,

Sinorhizobium meliloti, 
Streptomyces

pristinaespiralis,

Streptomyces prunicolor,

Streptomyces rimosus

subsp. rimosus,

Streptomyces

roseosporus,

Streptomyces

scabrisporus,

Streptomyces

somaliensis,

Streptomyces sulphureus,

Streptomyces sviceus,

Streptomyces

tsukubaensis,

Streptomyces

turgidiscabies,

Streptomyces

viridochromogenes,

Streptomyces

viridosporus,

Streptomyces

vitaminophilus,

Streptomyces

zinciresistens,

Amycolatopsis azurea,

Amycolatopsis

decaplanina,

Amycolatopsis

methanolica,

Saccharopolyspora spi-

nosa,

Nocardia abscessus,

Nocardia aobensis,

Nocardia araoensis,
Rhodococcus jostii, Blas-

tococcus saxobsidens,

Beutenbergia cavernae,

Streptomyces collinus,

Arthrobacter phenan-

threnivorans, Arthrobacter

chlorophenolicus, Xan-

thomonas campestris $p v$.

raphani, Xylanimonas cel-

Iulosilytica, Thermobispora

bispora, Sinorhizobium

medicae, Sanguibacter

keddieii, Sinorhizobium

meliloti, Ramlibacter ta-

taouinensis, Intrasporan-

gium calvum 
Nocardia asiatica,

Nocardia asteroides,

Nocardia brasiliensis,

Nocardia brevicatena,

Nocardia carnea,

Nocardia cerradoensis,

Nocardia concava,

Nocardia cyriacigeorgica,

Nocardia exalbida,

Nocardia higoensis,

Nocardia jiangxiensis,

Nocardia niigatensis,

Nocardia otitidiscaviarum,

Nocardia paucivorans,

Nocardia pneumoniae,

Nocardia takedensis,

Nocardia tenerifensis,

Nocardia terpenica,

Nocardia testacea,

Nocardia thailandica,

Nocardia veterana,

Nocardia vinacea,

Rhodococcus erythropolis,

Rhodococcus imte-

chensis,

Rhodococcus opacus,

Rhodococcus pyridinivo-

rans,

Rhodococcus qingshengii,

Rhodococcus rhodo-

chrous,

Rhodococcus ruber,

Rhodococcus triatomae,

Rhodococcus wrati-

slaviensis, 
Smaragdicoccus niigaten-

sis,

Mycobacterium leprae,

Mycobacterium tuberculo-

sis

Mycobacterium abscessus

subsp. bolletii,

Mycobacterium ab-

scessus,

Mycobacterium avium

subsp. avium,

Mycobacterium canettii,

Mycobacterium colombi-

ense,

Mycobacterium fortuitum

subsp. fortuitum,

Mycobacterium hassi-

acum,

Mycobacterium massili-

ense,

Mycobacterium parascrof-

ulaceum,

Mycobacterium phlei,

Mycobacterium rhodesiae,

Mycobacterium smegma-

tis,

Mycobacterium ther-

moresistibile,

Mycobacterium tusciae,

Mycobacterium vaccae,

Mycobacterium xenopi

Streptomyces albus, Ligase

Streptomyces carneus,

Streptomyces avermitilis,

Streptomyces

Mycobacterium tuberculosis H37Rv, Mycobacte-

bingchenggensis,

rium abscessus, Mycobac- 
Streptomyces coelicolor,

Streptomyces pratensis,

Streptomyces

rapamycinicus,

Streptomyces scabiei,

Streptomyces venezuelae,

Streptomyces

violaceusniger,

Frankia symbiont of Da-

tisca glomerata,

Rhodococcus equi,

Frankia symbiont of Datisca glomerata,

Rhodococcus equi, terium canettii, Mycobac-

terium mageritense, $M y$ cobacterium farcinogenes, Mycobacterium spp., Rhodococcus erythropolis, Rhodococcus equi, Rhodococcus fascians, Rhodococcus rhodochrous, Rhodococcus pyridinivorans, Rhodococcus rhodnii, Rhodococcus spp., Nocardia araoensis, Nocardia transvalensis, Nocardia exalbida, Nocardia spp., Gordonia polyisoprenivorans, Gordonia spp., Smaragdicoccus niigatensis,

Primase and Polymerase Streptomyces carneus, Mycobacterium tuberculosis H37Rv, Mycobacterium canettii, Mycobacterium orygis, Mycobacterium spp., Rhodococcus erythropolis, Rhodococcus equi, Rhodococcus ruber, Rhodococcus pyridinivorans, Rhodococcus fascians, Rhodococcus rhodochrous, Rhodococcus fascians Rhodococcus spp., Nocardia thailandica, Nocardia exalbida, Nocardia asteroides, Nocardia vinacea, Nocardia spp. Amy- 
Streptomyces scabiei DNA-binding colicicoccus subflavus, Tomitella biformata, Smaragdicoccus niigatensis Mycobacterium tuberculosis H37Rv, Mycobacterium africanum, Mycobacterium canettii, Mycobacterium spp. Streptomyces coelicolor, Streptomyces cattleya, Streptomyces purpureus, Streptomyces varsoviensis, Streptomyces thermolilacinus, Streptomyces roseoverticillatus, Streptomyces venezuelae, Streptomyces spp. Amycolatopsis mediterranei, Amycolatopsis halophila, Amycolatopsis vancoresmycina, Amycolatopsis orientalis, Amycolicicoccus subflavus, Amycolatopsis spp., Nakamurella multipartita, Beutenbergia cavernae, Arthrobacter castelli, Saxeibacter lacteus, Rhodococcus equi, Nocardia jiangxiensis, Gordonia rubripertincta, Clavibacter michiganensis, Gordonia aichiensis, Microbacterium paraoxydans 
In one embodiment, the host cell is $S$. coelicolor. This organism lacks the ligase activity of the NHEJ pathway and only displays the DNA-binding activity via the Ku proteins and the primase and polymerase activity (SEQ ID NO: 70). In one embodiment, NHEJ is restored in S. coelicolor by introducing at least part of the ligD gene from $S$. carneus, wherein said part encodes the ligase activity. In other embodiments, NHEJ is restored by introducing the ligD gene from $M$. tuberculosis, Nocardia spp., Smaragdicoccus niigatensis, Rhodococcus spp., Mycobacterium abscessus, Mycobacterium mageritense or Mycobacterium farcinogenes.

\section{Target nucleic acid}

The method disclosed herein is particularly useful for generating precise indels around at least one target nucleic acid sequence of interest. The method is thus useful for, but not limited to, the investigation of pathway regulations and the identification of metabolite production bottlenecks, the screening of producer strains and the identification of new compounds produced by the host cell.

The target nucleic acid sequence may be comprised within any nucleic acid sequence of interest. For example, the target sequence may be comprised within or may comprise an open reading frame or a putative open reading frame, or it may be comprised within or may comprise a regulatory region or a putative regulatory region, such as an enhancer, a promoter, an insulator, a terminator.

The target nucleic acid sequence may be involved in a pathway of interest. In some embodiments, the target nucleic acid encodes an enzyme or a protein. In other embodiments, the target nucleic acid is comprised within or comprises a biosynthetic gene or a putative biosynthetic gene. In some embodiments, the biosynthetic gene is involved in the synthesis of a secondary metabolite.

In some embodiments, the target nucleic acid sequence is comprised within a gene cluster. In specific embodiments, the gene cluster is a secondary metabolite gene cluster.

There is thus disclosed herein a method for generating precise indels such at precise deletions or precise insertions around a target nucleic acid sequence optionally comprised within or comprising a gene cluster, where the target nucleic acid sequence is 
involved or is suspected of being involved in the biosynthesis of a secondary metabolite.

In some embodiments, the secondary metabolite is selected from the group consisting of antibiotics, herbicides, anti-cancer agents, immunosuppressants, flavors, parasiticides and proteins. The term 'parasiticide' is to be understood in its broadest sense as an agent capable of inactivating or killing any undesirable organism and thus comprises insecticides, anthelmintic compounds, larvacides, antiparasitic agents and antiprotozoal agents.

10

In some embodiments, the secondary metabolite is an antibiotic selected from the group consisting of apramycin, bacitracin, chloramphenicol cephalosporins, cycloserine, erythromycin, fosfomycin, gentamicin, kanamycin, kirromycin, lassomycin, lincomycin, lysolipin, microbisporicin, neomycin, noviobiocin, nystatin, nitrofurantoin, 15 platensimycin, pristinamycins, rifamycin, streptomycin, teicoplanin, tetracycline, tinidazole, ribostamycin, daptomycin, vancomycin, viomycin and virginiamycin.

In other embodiments, the secondary metabolite is a herbicide selected from the group consisting of bialaphos, resormycin and phosphinothricin.

20

In yet other embodiments, the secondary metabolite is an anti-cancer agent selected from the group consisting of doxorubicin, salinosporamides, aclarubicin, pentostatin, peplomycin, thrazarine and neocarcinostatin.

25 In yet other embodiments, the secondary metabolite is an immunosuppressant selected from the group consisting of rapamycin, FK520, FK506, cyclosporine, ushikulides, pentalenolactone I and hygromycin A.

In yet other embodiments, the secondary metabolite is a flavor such as geosmin.

In yet other embodiments, the secondary metabolite is a parasiticide such as an insecticide, an anthelmintic, a larvacide, or an antiprotozoal agent such as spinsad or avermectin. 
In other embodiments, the target nucleic acid encodes an enzyme such as a metabolic enzyme selected from the group consisting of an amylase, a protease, a cellulase, a chitinase, a keratinase and a xylanase, a glycosyltransferase, an oxygenase, a hydroxylase, a methyltransferase, a dehydrogenase, a dehydratase.

5

In some embodiments, only one target nucleic acid sequence is targeted for editing and generation of precise indels. In other embodiments, more than one target nucleic acid sequence is targeted and the method is a multiplex method. Thus the method can be used for generating at least one indel around at least one target nucleic acid sequence, such as at least two indels around at least two target nucleic acid sequences, such as at least three indels around at least three target nucleic acid sequences, such as at least four indels around at least four target nucleic acid sequences, such as at least five indels around at least five target nucleic acid sequences, or more. The method can thus be used for generating one indel around one target nucleic acid sequence, or two indels around at least two target nucleic acid sequences, or three indels around three target nucleic acid sequences, or four indels around four target nucleic acid sequences, or five indels around five target nucleic acid sequences, or more. As explained above, in the case of multiplex editing, a guiding means is preferably provided for each target nucleic acid sequence.

20

In some embodiments, the at least one indel results in the inactivation of at least one gene. In some embodiments, the at least one gene is comprised within a gene cluster. In other embodiments, the at least one gene is not comprised within a gene cluster.

25 The at least one indel generated by the present method is an indel of at least $1 \mathrm{bp}$.

\section{Efficiency and off-target effects}

Several parameters can have an impact on the efficiency of the present method for generating precise indels around at least one target sequence. Some parameters can be adjusted as known in the art. Parameters susceptible of having an impact on the efficiency include, but are not limited to: the sequence of the guiding means (sgRNA or crRNA/tracrRNA), the sequence of the target nucleic acid, the GC content of the host cell and the GC content of the target nucleic acid sequence. 
The method for generating precise indels around a target nucleic acid sequence described herein can be performed with high efficiency, with relatively few off-target effects. In some embodiments, the desired indel is generated in more than $65 \%$ of the host cells, such as in more than $70 \%$ of the host cells, such as in more than $75 \%$ of the host cells, such as in more than $80 \%$ of the host cells, such as in more than $85 \%$ of the host cells, such as in more than $90 \%$ of the host cells, such as in more than $95 \%$ of the host cells, such as in $100 \%$ of the host cells.

Without being bound by theory, the use of homology arms to direct the repair of the break generated by the Cas 9 nuclease towards the HR pathway is believed to reduce the occurrence of off-target effects. When homology arms are used, higher efficiency can be achieved, so that the desired indel is generated in more than $90 \%$ of the host cells, such as in more than $95 \%$ of the host cells, such as in more than $96 \%$ of the host cells, such as in more than $97 \%$ of the host cells, such as in more than $98 \%$ of the host cells, such as in more than $99 \%$ of the host cells, such as in $100 \%$ of the host cells.

\section{Characterisation and screening}

The present method can thus be used for generating precise indels around a target nucleic acid sequence of interest, for example a sequence encoding for a gene involved in a pathway of interest. This can result in a plurality of clones having precise indels around the target sequence. These clones can then be further analysed or screened. For example, producer strains having advantageous production profiles for a desired compound can be selected.

In some embodiments, it may be of interest to determine the size of the at least one indel for a particular clone. Thus the method may comprise a further step of determining the size of the at least one indel. Methods for determining the size of an indel are known in the art and include, but are not limited to, whole genome sequencing, pulsed field gel electrophoresis, nucleic acid amplification-based methods such as PCR, for example followed by restriction analysis and detection of the PCR products on a gel and determination of the size of the products using an appropriate marker. The PCR products can also be sequenced if precise determination of the size of the indel is desired. 
In some embodiments, the method further comprises the selection of clones having the desired characteristics. Such selection methods are known in the art and encompass screening methods, chemical analysis of the related gene products (proteins or metabolites), sequencing of the related gene regions, and/or analysis of the gene expression level.

\section{CRISPR-Cas 9 system for actinomycetes}

The most studied CRISPR-Cas9 system is from Streptococcus pyogenes, which has a GC content of about $35 \%$. In contrast, actinomycetes have a high GC content. S. coelicolor for example has a GC content of about $72 \%$. Likewise, codon usage varies from organism to organism.

Herein is thus disclosed a codon optimised nucleic acid sequence encoding Cas 9 which is codon optimised for streptomycetes (SEQ ID NO: 1). The optimisation was

15 done based on the codon usage table of the most studied actinomycete, Streptomyces coelicolor, as described in example 1.

In one aspect, the invention thus relates to a polynucleotide having at least $94 \%$ identity with SEQ ID NO: 1 , such as at least $95 \%$ identity, such as at least $96 \%$ identity, such 20 as at least $97 \%$ identity, such as at least $98 \%$ identity, such as at least $99 \%$ identity, such as $100 \%$ identity, said polynucleotide encoding a Cas 9 nuclease or a variant thereof. It will be understood that sequences closely related to SEQ ID NO: 1 with mutations such as e.g. silent mutations are envisaged.

In some embodiments, the polynucleotide is non-naturally occurring.

Also within the scope of the present disclosure is a polypeptide encoded by a polynucleotide having at least $94 \%$ identity with SEQ ID NO: 1, such as at least $95 \%$ identity, such as at least $96 \%$ identity, such as at least $97 \%$ identity, such as at least $98 \%$ identity, such as at least $99 \%$ identity, such as $100 \%$ identity with SEQ ID NO: 1 . In one embodiment, the polypeptide has the sequence as set forth in SEQ ID NO: 2.

It will be understood that sequences closely related to SEQ ID NO: 2 with mutations that do not disrupt the function of Cas9 are also within the scope of the invention. In particular, mutations in non-conserved domains of Cas 9 which are unlikely to affect its 
function and conservative mutations in conserved or non-conserved domains of Cas 9 are envisaged.

In some embodiments, the polypeptide is non-naturally occurring.

Also within the scope of the present disclosure is a cell comprising the polynucleotide disclosed herein. Such a cell may be a host cell as detailed above. In particular, the cell may be an archaea, in a prokaryotic cell or in a eukaryotic cell. In one embodiment, the host cell is a prokaryotic cell. The host cell may be a cell with a high GC content, for 10 example a GC content of $50 \%$ or more, such as $55 \%$ or more, such as $60 \%$ or more, such as $65 \%$ or more, such as $70 \%$ or more, such as $75 \%$ or more, such as $80 \%$ or more, such as $85 \%$ or more, such as $90 \%$ or more. In a particular embodiment, the host cell is an actinobacterium. The host cell may thus be selected from the group consisting of Actinomycetales, such as Streptomyces sp., Amycolatopsis sp. or Saccharopolyspora $s p$. In some embodiments, the host cell is selected from the group consisting of Streptomyces coelicolor, Streptomyces avermitilis, Streptomyces aureofaciens, Streptomyces griseus, Streptomyces parvulus, Streptomyces albus, Streptomyces vinaceus, Streptomyces acrimycinis, Streptomyces calvuligerus, Streptomyces lividans, Streptomyces limosus, Streptomyces rubiqinosis, Streptomyces azureus, Streptomyces glaucenscens, Streptomyces rimosus, Streptomyces violaceoruber, Streptomyces kanamyceticus, Amycolatopsis orientalis, Amycolatopsis mediterranei, Saccharopolyspora erythraea, Mycobacterium tuberculosis, Streptomyces carneus, Nocardia spp., Smaragdicoccus niigatensis, Rhodococcus spp., Mycobacterium abscessus, Mycobacterium mageritense, Mycobacterium farcinogenes. In a preferred embodiment, the host cell is Streptomyces coelicolor.

The present disclosure also relates to a vector comprising the polynucleotide as described herein. Thus some embodiments relate to a vector comprising a polynucleotide having at least $94 \%$ identity with SEQ ID NO: 1 , such as at least $95 \%$ identity, such as at least $96 \%$ identity, such as at least $97 \%$ identity, such as at least $98 \%$ identity, such as at least $99 \%$ identity, such as $100 \%$ identity with SEQ ID NO: 1.

The polynucleotide, the polypeptide and/or the vector comprising the polynucleotide, as all disclosed herein, may be used for performing the methods disclosed herein. In pre- 
ferred embodiments, they are used to perform the present methods in a host cell, where the host cell is a Streptomycetes.

In some embodiments, the method is a method for generating at least one deletion around at least one target nucleic acid sequence comprised within a host cell having a non-homologous end-joining (NHEJ) pathway which is at least partly deficient, said method comprising the steps of:

(i) optionally, restoring the full functionality of the NHEJ pathway,

(ii) inducing a CRISPR-Cas9 system in said host cell, wherein said CRISPR-Cas 9 system is able to generate at least one break in said at least one target nucleic acid sequence and wherein the CRISPRCas9 system comprises a Cas9 nuclease and at least one guiding means,

thereby generating:

a. if the method does not comprise step (i), at least one random-sized deletion around said at least one target nucleic acid sequence, wherein said at least one deletion is a random-sized deletion of at least $1 \mathrm{bp}$; or

b. if the method does comprise step (i), at least one indel around said at least one target nucleic acid sequence, wherein said at least one indel is a deletion or insertion of at least $1 \mathrm{bp}$,

wherein Cas 9 is a polypeptide as described above, or wherein Cas 9 is encoded by a polynucleotide as described above.

25 Accordingly, in some embodiments, the method does not comprise step (i) of restoring the full functionality of the NHEJ pathway and results in generation of random-sized deletions, where Cas 9 is a polypeptide encoded by a polynucleotide having at least $94 \%$ identity with SEQ ID NO: 1 , such as at least $95 \%$ identity, such as at least $96 \%$ identity, such as at least $97 \%$ identity, such as at least $98 \%$ identity, such as at least $99 \%$ identity, such as $100 \%$ identity with SEQ ID NO: 1 . In one embodiment, the polypeptide has the sequence as set forth in SEQ ID NO: 2. In some embodiments, the polynucleotide encoding Cas 9 is codon-optimised for the host cell in which the method is to be performed. 
In other embodiments, the method comprises step (i) of restoring the full functionality of the NHEJ pathway and results in generation of indels, i.e. insertions of deletions of at least $1 \mathrm{bp}$, where Cas 9 is a polypeptide encoded by a polynucleotide having at least $94 \%$ identity with SEQ ID NO: 1 , such as at least $95 \%$ identity, such as at least $96 \%$ identity, such as at least $97 \%$ identity, such as at least $98 \%$ identity, such as at least $99 \%$ identity, such as $100 \%$ identity with SEQ ID NO: 1 . In one embodiment, the polypeptide has the sequence as set forth in SEQ ID NO: 2. In some embodiments, the polynucleotide encoding Cas 9 is codon-optimised for the host cell in which the method is to be performed.

10

\section{Method for selective modulation of transcription}

In another aspect, a method for selectively modulating transcription of at least one target nucleic acid sequence in a host cell is disclosed, the method comprising introducing into the host cell:

i. at least one guiding means, or a nucleic acid comprising a nucleotide sequence encoding guiding means, wherein the guiding means comprises a nucleotide sequence that is complementary to a target nucleic acid sequence in the host cell; and

ii. a variant Cas9, or a nucleic acid comprising a nucleotide sequence encoding the variant Cas9, wherein the variant Cas 9 has reduced endodeoxyribonuclease activity,

wherein said guiding means and said variant Cas 9 form a complex in the host cell, said complex selectively modulating transcription of at least one target nucleic acid in the host cell.

In some embodiments, the method for selectively modulating transcription of at least one target nucleic acid sequence in a host cell comprises introducing into the host cell:

(i) at least one guiding means, or a nucleic acid comprising a nucleotide sequence encoding guiding means, wherein the guiding means comprises a nucleotide sequence that is complementary to a target nucleic acid sequence in the host cell; and

(ii) a variant Cas9, or a nucleic acid comprising a nucleotide sequence encoding the variant Cas9, wherein the variant Cas9 is a variant of the polypeptides disclosed herein or of a polypeptide encoded by the nucleotide sequences disclosed herein, and wherein the variant Cas 9 
has reduced endodeoxyribonuclease activity, with reduced endodeoxyribonuclease activity and is codon-optimised for Streptomycetes, wherein said guiding means and said variant Cas9 form a complex in the host cell, said complex selectively modulating transcription of at least one target nucleic acid in the host cell.

In some embodiments, the guiding means comprises at least one sgRNA and/or at least one crRNA/tracrRNA set.

10

\section{Modulation}

This method allows selective modulation of the transcription of at least one target nucleic acid sequence comprised within a host cell.

15 Modulation of the transcription can be an increase of the transcription level or a decrease of the transcription level.

The method for modulation of transcription is based on the use of a CRISPR-Cas 9 system comprising a variant Cas 9 and at least one guiding means, wherein the variant

20 Cas 9 is capable of forming a complex with each of the at least one guiding means and is thereby capable of binding to the target nucleic acid sequence but is not capable of inducing a break therein or is not capable of leaving the target nucleic acid sequence. In other words, variant Cas9 remains on the target nucleic acid sequence, whereby it is hypothesized that transcription is prevented because of steric hindrance or lower accessibility of a polymerase such as an RNA polymerase to the DNA. In order to achieve an increase of transcription, a transcription activator can be fused to the variant Cas9, wherein the variant Cas 9 is capable of forming a complex with at least one guiding means targeting e.g. the promoter of a gene of interest; the complex remains on the target nucleic acid sequence and thereby provides a transcription activator, thereby activating expression of the gene.

In some embodiments, the variant Cas9 is a variant Cas9 which can cleave one of the strands of the target nucleic acid sequence but has reduced ability to cleave the other strand of the target nucleic acid sequence. In some embodiments, the variant Cas 9 is selected from the group consisting of Cas9-H840A, Cas9-D10A and Cas9-H840A, 
D10A, where H840A indicates a substitution at amino acid residue 840 of SEQ ID NO: 2 , and D10A indicates a substitution at amino acid residue 10 of Cas9. It will be understood that sequences having mutations that do not disrupt the function of the variant Cas 9 are also within the scope of the invention. In particular, mutations in nonconserved domains of Cas 9 which are unlikely to affect its function and conservative mutations in conserved or non-conserved domains of Cas 9 are envisaged.

In some embodiments, the expression of the variant Cas 9 is inducible, e.g. the nucleic acid sequence encoding the variant Cas 9 may be under the control of an inducible promoter. Other methods of inducing expression of the variant Cas9 will be apparent to the skilled person.

In some embodiments, the nucleic acid sequence encoding the variant Cas9 is comprised within a vector to be introduced in the host cell. In other embodiments, the nucleic acid sequence encoding the variant Cas 9 is comprised within the genome of the host cell, e.g. on a chromosome.

The CRISPR-Cas9 system preferably further comprises at least one guiding means allowing the variant Cas9 to bind to the at least one target nucleic acid sequence and to modulate its transcription. As detailed above, the nucleic acid sequence encoding the variant Cas 9 and the at least one nucleic acid sequence encoding the at least one guiding means may be comprised within a single nucleic acid such as a vector or a chromosome comprised within the host cell.

Host cell

The present method can be performed in an archaea, in a prokaryotic cell or in a eukaryotic cell. In one embodiment, the host cell is a prokaryotic cell. The present methods are particularly advantageous for modulating transcription in host cells that have a high GC content, for example a GC content of $50 \%$ or more, such as $55 \%$ or more, such as $60 \%$ or more, such as $65 \%$ or more, such as $70 \%$ or more, such as $75 \%$ or more, such as $80 \%$ or more. In a particular embodiment, the host cell is an actinobacterium. The host cell may thus be selected from the group consisting of Actinomycetales, such as Streptomyces sp., Amycolatopsis sp. or Saccharopolyspora sp. In some embodiments, the host cell is selected from the group consisting of Streptomyces coelicolor, Streptomyces avermitilis, Streptomyces aureofaciens, Streptomyces 
griseus, Streptomyces parvulus, Streptomyces albus, Streptomyces vinaceus, Streptomyces acrimycinis, Streptomyces calvuligerus, Streptomyces lividans, Streptomyces limosus, Streptomyces rubiqinosis, Streptomyces azureus, Streptomyces glaucenscens, Streptomyces rimosus, Streptomyces violaceoruber, Streptomyces kanamyceticus, Amycolatopsis orientalis, Amycolatopsis mediterranei, Saccharopolyspora erythraea, Mycobacterium tuberculosis, Streptomyces carneus, Nocardia spp., Smaragdicoccus niigatensis, Rhodococcus spp., Mycobacterium abscessus, Mycobacterium mageritense, Mycobacterium farcinogenes. In a preferred embodiment, the host cell is Streptomyces coelicolor.

10

The host cell may be any of the organisms listed herein elsewhere.

\section{Target nucleic acid}

The method disclosed herein is particularly useful for modulating transcription of least one target nucleic acid sequence of interest. The method is thus useful for, but not limited to, the investigation of pathway regulations and identification of metabolite production bottlenecks, the design of producer strains and the identification of new compounds produced by the host cell.

20 The target nucleic acid sequence may be comprised within any nucleic acid sequence of interest. For example, the target sequence may be comprised within or may comprise an open reading frame or a putative open reading frame, or it may be comprised within or may comprise a regulatory region or a putative regulatory region, such as an enhancer, a promoter, an insulator, a terminator.

The target nucleic acid sequence may be involved in a pathway of interest. In some embodiments, the target nucleic acid encodes an enzyme. In other embodiments, the target nucleic acid is comprised within or comprises a biosynthetic gene or a putative biosynthetic gene. In some embodiments, the biosynthetic gene is involved in the synthesis of a secondary metabolite.

In some embodiments, the target nucleic acid sequence is comprised within a gene cluster. In specific embodiments, the gene cluster is a secondary metabolite gene cluster. 
There is thus disclosed herein a method for modulating transcription of at least one target nucleic acid sequence optionally comprised within or comprising a gene cluster, where the target nucleic acid sequence is involved or is suspected of being involved in the biosynthesis of a secondary metabolite.

In some embodiments, the secondary metabolite is selected from the group consisting of antibiotics, herbicides, anti-cancer agents, immunosuppressants, flavors, parasiticides, enzymes and proteins. The term 'parasiticide' is to be understood in its broadest sense as an agent capable of inactivating or killing any undesirable organism and thus comprises insecticides, anthelmintic compounds, larvacides, antiparasitic agents and antiprotozoal agents.

In some embodiments, the secondary metabolite is an antibiotic selected from the group consisting of apramycin, bacitracin, chloramphenicol cephalosporins, cycloserine, erythromycin, fosfomycin, gentamicin, kanamycin, kirromycin, lassomycin, lincomycin, lysolipin, microbisporicin, neomycin, noviobiocin, nystatin, nitrofurantoin, platensimycin, pristinamycins, rifamycin, streptomycin, teicoplanin, tetracycline, tinidazole, ribostamycin, daptomycin, vancomycin, viomycin and virginiamycin.

20 In other embodiments, the secondary metabolite is a herbicide selected from the group consisting of bialaphos, resormycin and phosphinothricin.

In yet other embodiments, the secondary metabolite is an anti-cancer agent selected from the group consisting of doxorubicin, salinosporamides, aclarubicin, pentostatin, peplomycin, thrazarine and neocarcinostatin.

In yet other embodiments, the secondary metabolite is an immunosuppressant selected from the group consisting of rapamycin, FK520, FK506, cyclosporine, ushikulides, pentalenolactone I and hygromycin A.

In yet other embodiments, the secondary metabolite is a flavor such as geosmin.

In yet other embodiments, the secondary metabolite is a parasiticide such as an insecticide, an anthelmintic, a larvacide, or an antiprotozoal agent such as spinsad or avermectin. 
In other embodiments, the target nucleic acid encodes an enzyme such as metabolic enzyme selected from the group consisting of an amylase, a protease, a cellulase, a chitinase, a keratinase and a xylanase, a glycosyltransferase, an oxygenase, a hydroxylase, a methyltransferase, a dehydrogenase, a dehydratase.

In some embodiments, transcription of only one target nucleic acid sequence is modulated. In other embodiments, transcription of more than one target nucleic acid sequence is modulated and the method is a multiplex method. Thus the method can be used for modulating transcription of at least one target nucleic acid sequence, such as of least two target nucleic acid sequences, such as of at least three target nucleic acid sequences, such as of at least four target nucleic acid sequences, such as of at least five target nucleic acid sequences, or more. The method can thus be used for modulating transcription of one target nucleic acid sequence, of two target nucleic acid sequences, of three target nucleic acid sequences, of four target nucleic acid sequences, of five target nucleic acid sequences, or more. As explained above, in the case of multiplex modulation, a guiding means is preferably provided for each target nucleic acid sequence.

20 In some embodiments, the at least one nucleic acid sequence is at least one gene. The gene may be comprised within a gene cluster. In other embodiments, the at least one gene is not comprised within a gene cluster.

\section{Kits}

Kit for generating random-sized deletions and/or indels

In a further aspect, the disclosure relates to a kit for performing the methods described herein.

In some embodiments, the kit is for generating at least one random-sized deletion around at least one target nucleic acid sequence described above, said kit comprising a vector comprising a nucleic acid sequence encoding a Cas 9 nuclease or a variant thereof and instructions for use.

The vector comprised within said kit can be an integrative vector for integrating the nucleic acid sequence encoding the nuclease into the genome, or it can be comprised 
within a non-integrative vector, e.g. to be used as a template for amplifying the nucleic acid sequence encoding the nuclease prior to introduction into the cell, or to be transformed and maintained in the host cell.

5 In preferred embodiments, the nuclease is Cas9 or a variant thereof. In some embodiments, the nucleic acid sequence encoding the nuclease is a sequence encoding Cas 9 such as a polynucleotide having at least $93 \%$ identity with SEQ ID NO: 1 , such as at least $94 \%$ identity, such as at least $95 \%$ identity, such as at least $96 \%$ identity, such as at least $97 \%$ identity, such as at least $98 \%$ identity, such as at least $99 \%$ identity, such as $100 \%$ identity with SEQ ID NO: 1.

The kit may further comprise at least one guiding means and/or at least one host cell having a non-homologous end-joining (NHEJ) pathway which is at least partly deficient.

15 In some embodiments, the kit further comprises at least one guiding means, where the guiding means is as described above. The guiding means may be comprised within the vector or it may be provided on a different vector. The at least one guiding means may be any guiding means described above, such as an sgRNA or a crRNA/tracrRNA set.

20 In some embodiments, the kit further comprises a host cell or a plurality of host cells. In one embodiment, the host cell is a cell having a partly deficient NHEJ pathway, i.e. lacking at least one of the four NHEJ activities defined above. The host cell may be any of the host cells described herein elsewhere. The NHEJ pathway may be partly deficient because it is naturally partly deficient in said host cell, or it may have been inactivated by the manufacturer or by the user. In one embodiment, the host cell is S. coelicolor and lacks the ligase activity.

In other embodiments, the host cell has a functional NHEJ pathway. The kit may then further comprise means for at least partly inactivating the NHEJ pathway in said host cell. This can be done as described above, i.e. by inactivating at least one of the four NHEJ activities (DNA binding, ligase, polymerase or primase activity). Thus in one embodiment the kit comprises means for inactivating the ligase activity of the host cell.

In some embodiments, the kit is for performing the method for generating at least one precise indel around at least one target nucleic acid sequence, said kit comprising a 
first vector comprising a nucleic acid sequence encoding Cas9 or a variant thereof and instructions for use.

In some embodiments, the nucleic acid sequence encoding Cas9 is a polynucleotide having at least $93 \%$ identity with SEQ ID NO: 1 , such as at least $94 \%$ identity, such as at least $95 \%$ identity, such as at least $96 \%$ identity, such as at least $97 \%$ identity, such as at least $98 \%$ identity, such as at least $99 \%$ identity, such as $100 \%$ identity with SEQ ID NO: 1.

10 In some embodiments, the kit further comprises at least one guiding means, where the guiding means is as described above. The guiding means may be comprised within the first vector or it may be provided on a different vector. The at least one guiding means may be any guiding means described above, such as an sgRNA or a crRNA/tracrRNA set.

In some embodiments, the kit further comprises a host cell or a plurality of host cells. In one embodiment, the host cell is a cell having a partly deficient NHEJ pathway, i.e. lacking at least one of the four NHEJ activities defined above. The host cell may be any of the host cells described herein elsewhere. The NHEJ pathway may be partly defi-

20 cient because it is naturally partly deficient in said host cell, or it may have been inactivated by the manufacturer. In one embodiment, the host cell is $S$. coelicolor and lacks the ligase activity.

In other embodiments, the host cell has a functional NHEJ pathway. The kit may then 25 further comprise means for at least partly inactivating the NHEJ pathway in said host cell. This can be done as described above, i.e. by inactivating at least one of the four NHEJ activities (DNA binding, ligase, polymerase or primase activity). Thus in one embodiment the kit comprises means for inactivating the ligase activity of the host cell.

30 In some embodiments, the kit further comprises a second vector comprising a nucleic acid sequence encoding at least one of the four NHEJ activities defined above. In one embodiment, the nucleic acid thus encodes at least one of:

- a DNA-binding activity,

- a primase activity,

- a ligase activity, 
- a polymerase activitiy.

In some embodiments, the nucleic acid sequence encodes two or three of the four NHEJ activities. In some embodiments, the nucleic acid sequence encodes all four NHEJ activities. In some embodiments, the nucleic acid sequence encodes the ligase $D$ from S. carneus or $M$. tuberculosis. In a particular embodiment, the host cell is $S$. coelicolor and the nucleic acid sequence encoding the missing NHEJ activity comprises the ligase D gene from S. carneus or $M$. tuberculosis. Examples of which organisms having sequences that can be used for restoring NHEJ activity are provided above (Table 2).

In other embodiments, the nucleic acid sequence encoding at least one of the four $\mathrm{NEHJ}$ activities and the nucleic acid sequence encoding Cas9 are all comprised within the first vector.

\section{Kit for modulating transcription}

In yet another aspect is disclosed a kit for performing the method for modulating transcription of at least one target nucleic acid as described above, said kit comprising a vector comprising a nucleic acid sequence encoding a variant Cas 9 ; and instructions

20 for use. In preferred embodiments, the variant Cas9 has reduced endodeoxyribonuclease activity.

In some embodiments, the variant Cas9 is a variant Cas9 which can cleave one of the strands of the target nucleic acid sequence but has reduced ability to cleave the other strand of the target nucleic acid sequence. In some embodiments, the variant Cas 9 is selected from the group consisting of Cas9-H840A, Cas9-D10A and Cas9-H840A, D10A, where H840A indicates a substitution at amino acid residue 840 of SEQ ID NO: 2, and D10A indicates a substitution at amino acid residue 10 of Cas9. It will be understood that sequences having mutations that do not disrupt the function of the variant

30 Cas9 are also within the scope of the invention. In particular, mutations in nonconserved domains of Cas 9 which are unlikely to affect its function and conservative mutations in conserved or non-conserved domains of Cas 9 are envisaged.

In some embodiments, the kit further comprises at least one guiding means, where the guiding means is as described above, and/or at least one host cell or plurality of host 
cells. The guiding means may be comprised within the first vector or it may be provided on a different vector. The at least one guiding means may be any guiding means described above, such as an sgRNA or a crRNA/tracrRNA set.

5 The host cell may be an archaea, in a prokaryotic cell or in a eukaryotic cell. In one embodiment, the host cell is a prokaryotic cell. The present methods can be used for modulating transcription in host cells that have a high GC content, for example a GC content of $50 \%$ or more, such as $55 \%$ or more, such as $60 \%$ or more, such as $65 \%$ or more, such as $70 \%$ or more, such as $75 \%$ or more, such as $80 \%$ or more. In a particular embodiment, the host cell is an actinobacterium. The host cell may thus be selected from the group consisting of Actinomycetales, such as Streptomyces sp., Amycolatopsis sp. or Saccharopolyspora sp. In some embodiments, the host cell is selected from the group consisting of Streptomyces coelicolor, Streptomyces avermitilis, Streptomyces aureofaciens, Streptomyces griseus, Streptomyces parvulus, Streptomyces albus,

15 Streptomyces vinaceus, Streptomyces acrimycinis, Streptomyces calvuligerus, Streptomyces lividans, Streptomyces limosus, Streptomyces rubiqinosis, Streptomyces azureus, Streptomyces glaucenscens, Streptomyces rimosus, Streptomyces violaceoruber, Streptomyces kanamyceticus, Amycolatopsis orientalis, Amycolatopsis mediterranei, Saccharopolyspora erythraea, Mycobacterium tuberculosis, Streptomyces car-

20 neus, Nocardia spp., Smaragdicoccus niigatensis, Rhodococcus spp., Mycobacterium abscessus, Mycobacterium mageritense, Mycobacterium farcinogenes. In a preferred embodiment, the host cell is Streptomyces coelicolor.

\section{Examples}

\section{Example 1: Materials and methods}

Strains and chemicals

ISP2: Yeast Extract, 0.4\%, Malt Extract, 1\%, Dextrose, 0.4\%, 2\% agar for solidification, $\mathrm{pH}$ 7.2. Cullum agar, also termed SFM (soya flour mannitol) agar: $2 \%$ organic soya flour (low fat), $2 \%$ mannitol, $2 \%$ agar, $10 \mathrm{mM} \mathrm{MgCl}_{2}$, natural $\mathrm{pH}$. LB: Tryptone, $1 \%$, Yeast Extract, $0.5 \%, \mathrm{NaCl}, 0.5 \%, \mathrm{pH}, 7.0 .2 \times \mathrm{YT}$ : Tryptone, $1.6 \%$, Yeast Extract, $1 \%$, $\mathrm{NaCl}, 0.5 \%, \mathrm{pH} 7$. 
Chemicals and solutions: apramycin sulfate (stock solution $100 \mathrm{mg} / \mathrm{ml}$ in dd $\mathrm{H}_{2} \mathrm{O}$ ), nalidixic acid (stock solution $50 \mathrm{mg} / \mathrm{ml}$ in $\mathrm{ddH}_{2} \mathrm{O}$ of $\mathrm{pH}$ 11), thiostrepton (stock solution 50 $\mathrm{mg} / \mathrm{ml}$ in DMSO), kanamycin (stock solution $50 \mathrm{mg} / \mathrm{ml}$ in $\mathrm{dd}_{2} \mathrm{O}$ ), chloramphenicol (stock solution $50 \mathrm{mg} / \mathrm{ml}$ in ethanol), chloroform, methanol, and DMSO. The working concentrations for apramycin, nalidixic acid, thiostrepton, kanamycin, and chloramphenicol were $50 \mu \mathrm{g} / \mathrm{ml}, 50 \mu \mathrm{g} / \mathrm{ml}, 1 \mu \mathrm{g} / \mathrm{ml}, 25 \mu \mathrm{g} / \mathrm{ml}$, and $25 \mu \mathrm{g} / \mathrm{ml}$, respectively.

The below tables list selected target sequences (Table 3), primers (Table 4) and strains and plasmids (table 5) used in the following examples.

Table 3. Selected target sequences

\begin{tabular}{|c|c|c|c|}
\hline sgRNA & The target Sequences & PAM & Purpose \\
\hline Actlorf1-1 NT & GTGGCTCGAAGGAGGCTCGA & AGG & $\begin{array}{l}\text { Gene deletion/ ex- } \\
\text { pression control }\end{array}$ \\
\hline Actlorf1-2 T & AGCTCGATCAAGTCGATGGT & CGG & $\begin{array}{l}\text { Gene deletion/ ex- } \\
\text { pression control }\end{array}$ \\
\hline Actlorf1-3 T & GAAGCGCAGAGTCGTCATCA & CGG & $\begin{array}{l}\text { Gene deletion/ ex- } \\
\text { pression control }\end{array}$ \\
\hline Actlorf1-4 T & CCCCTCGCCCTACCGTTCAC & AGG & $\begin{array}{l}\text { Gene deletion/ ex- } \\
\text { pression control }\end{array}$ \\
\hline Actlorf1-5 T & GCGCGAGTATCTGCTGCTGT & CGG & Gene deletion \\
\hline Actlorf1-6 T & CTGCAACGCGTACCACATGA & CGG & Gene deletion \\
\hline Actvb-1 NT & TCGCCGCAACTGTCGAACAC & CGG & Gene deletion \\
\hline Actvb-2 NT & CTGCCATCTTCGAACTCCCT & $A G G$ & Gene deletion \\
\hline Actvb-3 T & TTCCCGGTGTTCGACAGTTG & CGG & Gene deletion \\
\hline Actvb-4 T & ACTGGTCTGCCTGGCTCGTA & CGG & Gene deletion \\
\hline Actvb-5 NT & ATCTTCGAACTCCCTAGGCG & AGG & Gene deletion \\
\hline Actvb-6 NT & GTCCCGGAGCATTCCCTGGT & CGG & Gene deletion \\
\hline orf1p-S1 T & GTGTTCCCCTCCCTGCCTCG & TGG & $\begin{array}{l}\text { Gene expression con- } \\
\text { trol }\end{array}$ \\
\hline orf1p-S3 T & TCCCTCACGCGCTCAGCTTT & GGG & $\begin{array}{l}\text { Gene expression con- } \\
\text { trol }\end{array}$ \\
\hline orf1p-S5 T & CTTTGGGCGCCCGGCTCGAG & CGG & Gene expression con- \\
\hline
\end{tabular}


trol

orf1p-A1 NT CCTTCGACCGCCGCTCGAGC CGG Ger

Gene expression control

orf1p-A4 NT GCCCAAAGCTGAGCGCGTGA AGG Gene expression control

orf1p-A5 NT TGAGCGCGTGAGGGACCACG AGG Gene expression control

Actlorf1-7 NT TGAGCAGTTCCCAGAACTGC CGG Gene expression control

Actlorf1-8 NT AGGAGGCTCGAAGGCCGATA CGG Gene expression control

Table 4. Primer list.

\begin{tabular}{|c|c|c|c|}
\hline Sets & Primer name & Sequence $\left(5^{\prime}-3^{\prime}\right)$ \# *§ & Purpose \\
\hline 1 & Actlorf1-F1 & $\begin{array}{l}\text { CATGCCATGGGTGGCT } \\
\text { CGAAGGAGGCTCGA } \\
\text { GTTTTAGAGCTAGAAATAGC }\end{array}$ & sgRNAs Amplification \\
\hline 2 & Actlorf1-F2 & $\begin{array}{l}\text { CATGCCATGGAGCTCG } \\
\text { ATCAAGTCGATGGTGT } \\
\text { TTTAGAGCTAGAAATAGC }\end{array}$ & \\
\hline 3 & Actlorf1-F3 & $\begin{array}{l}\text { CATGCCATGGGAAGCG } \\
\text { CAGAGTCGTCATCAGTT } \\
\text { TTAGAGCTAGAAATAGC }\end{array}$ & \\
\hline 4 & Actlorf1-F4 & $\begin{array}{l}\text { CATGCCATGGCCCCTCG } \\
\text { CCCTACCGTTCACGTTTT } \\
\text { AGAGCTAGAAATAGC }\end{array}$ & \\
\hline 5 & Actlorf1-F5 & $\begin{array}{l}\text { CATGCCATGGGCGCGA } \\
\text { GTATCTGCTGCTGTGTT } \\
\text { TTAGAGCTAGAAATAGC }\end{array}$ & \\
\hline 6 & Actlorf1-F6 & $\begin{array}{l}\text { CATGCCATGGCTGCAAC } \\
\text { GCGTACCACATGAGTT } \\
\text { TTAGAGCTAGAAATAGC }\end{array}$ & \\
\hline 7 & Actlorf1-F7 & $\begin{array}{l}\text { CATGCCATGGTGAGCA } \\
\text { GTTCCCAGAACTGCGTT }\end{array}$ & \\
\hline
\end{tabular}




\begin{tabular}{|c|c|c|}
\hline 8 & Actlorf1-F8 & CATGCCATGGAGGAGGC1 \\
\hline & & CGAAGGCCGATAGTT \\
\hline 9 & ActVB-F1 & CATGCCATGGTCGCCG \\
\hline & & CAACTGTCGAACACGTT \\
\hline & & TTAGAGCTAGAAATAGC \\
\hline 10 & ActVB-F2 & CATGCCATGGCTGCCAT \\
\hline & & CTTCGAACTCCCTGTT \\
\hline & & TTAGAGCTAGAAATAGC \\
\hline 11 & ActVB-F3 & CATGCCATGGTTCCCG \\
\hline & & GTGTTCGACAGTTGGTT \\
\hline & & TTAGAGCTAGAAATAGC \\
\hline 12 & ActVB-F4 & CATGCCATGGACTGGT \\
\hline & & CTGCCTGGCTCGTAGTT \\
\hline & & TTAGAGCTAGAAATAGC \\
\hline 13 & ActVB-F5 & CATGCCATGGATCTTCG \\
\hline & & AACTCCCTAGGCGGTT \\
\hline & & TTAGAGCTAGAAATAGC \\
\hline 14 & ActVB-F6 & CATGCCATGGGTCCCGG \\
\hline & & AGCATTCCCTGGTGTT \\
\hline & & TTAGAGCTAGAAATAGC \\
\hline 15 & orf1p-S1 T-F & 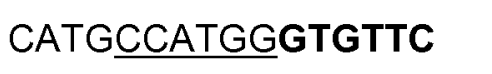 \\
\hline & & СсCTCCCTGCCTCGGTT \\
\hline & & TTAGAGCTAGAAATAGC \\
\hline 16 & orf1p-S3 T-F & CATGCCATGGTCCСTCA \\
\hline & & CGCGCTCAGCTTTGTT \\
\hline & & TTAGAGCTAGAAATAGC \\
\hline 17 & orf1p-S5 T-F & CATGCCATGGCTTTGG \\
\hline & & GCGCCCGGCTCGAGGTT \\
\hline & & TTAGAGCTAGAAATAGC \\
\hline 18 & orf1p-A1 NT-F & CATGCCATGGCCTTCG \\
\hline & & ACCGCCGCTCGAGCGTT \\
\hline & & TTAGAGCTAGAAATAGC \\
\hline 19 & orf1p-A4 NT-F & CATGCCATGGGCCCAAA \\
\hline & & GCTGAGCGCGTGAGTT \\
\hline & & TTAGAGCTAGAAATAGC \\
\hline
\end{tabular}




\begin{tabular}{|c|c|c|c|}
\hline 20 & orf1p-A5 NT-F & $\begin{array}{l}\text { CATGCCATGGTGAGCG } \\
\text { CGTGAGGGACCACGGTT } \\
\text { TTAGAGCTAGAAATAGC } \\
\text { ACGCCTACGTAAAAAAA } \\
\text { GCACCGACTCGGTGCC }\end{array}$ & \\
\hline $\begin{array}{l}22 \\
23\end{array}$ & $\begin{array}{l}\text { gRNA check-F } \\
\text { gRNA check-R }\end{array}$ & $\begin{array}{l}\text { ACATGTGCGGTCGATCTT } \\
\text { TACGTAAAAAAAGCACCGAC }\end{array}$ & sgRNAs sequencing \\
\hline $\begin{array}{l}26 \\
27\end{array}$ & $\begin{array}{l}\text { orf1-5'F } \\
\text { orf1-5'R } \\
\text { orf1-3'F } \\
\text { orf1-3'R }\end{array}$ & $\begin{array}{l}\text { TCGTCGAAGGCACTAGAAGG } \\
\text { CATCCGCTGAACGAGACCC } \\
\text { GCTCACGTCGAAGCGGGTG } \\
\text { ACCACGCAGGACTCCGAAGTC } \\
\text { TCACCCGCTTCGACGTGAG } \\
\text { GGTCGATCCCCGCATATAGG } \\
\text { TTCGCCGAGCACCAGGTC }\end{array}$ & $\begin{array}{l}\text { For actIORF1 homol- } \\
\text { ogous recombination } \\
\text { template construction }\end{array}$ \\
\hline $\begin{array}{l}30 \\
31\end{array}$ & $\begin{array}{l}\text { VB-5'F } \\
\text { VB-5'R } \\
\text { VB-3'F } \\
\text { VB-3'R }\end{array}$ & $\begin{array}{l}\text { TCGTCGAAGGCACTAGAAGG } \\
\text { CGACTCGCTCGCCCTGATG } \\
\text { CACCAACCTGCTCGGGCTG } \\
\text { CGCCGTGGAAGTGGGTGTTGAC } \\
\text { GCAGCCCGAGCAGGTTGG } \\
\text { GGTCGATCCCCGCATATAGG } \\
\text { TCCGTTGCGGCGTCCATC }\end{array}$ & $\begin{array}{l}\text { For actVB homolo- } \\
\text { gous recombination } \\
\text { template construction }\end{array}$ \\
\hline $\begin{array}{l}32 \\
33\end{array}$ & $\begin{array}{l}\text { VB-check-F } \\
\text { VB-check-R }\end{array}$ & $\begin{array}{l}\text { CGGCTGGTGCGTCAGCAAC } \\
\text { ACGTGGCGGGTCGAACGG }\end{array}$ & Check actVB deletion \\
\hline $\begin{array}{l}34 \\
35\end{array}$ & $\begin{array}{l}\text { ORF1-check-F } \\
\text { ORF1-check-R }\end{array}$ & $\begin{array}{l}\text { CCGCCTTGAGGACCTGTTTG } \\
\text { ACACGCTGACCGACTTGGG }\end{array}$ & $\begin{array}{l}\text { Check act/ORF1 dele- } \\
\text { tion }\end{array}$ \\
\hline $\begin{array}{l}36 \\
37\end{array}$ & $\begin{array}{l}\text { CAS9-check-F } \\
\text { CAS9-check-R }\end{array}$ & $\begin{array}{l}\text { TCCACGAGCACATCGCCAAC } \\
\text { GACCTTGTAGTCGCCGTAGACG }\end{array}$ & $\begin{array}{l}\text { Check cas9 sub- } \\
\text { cloning }\end{array}$ \\
\hline 36 & $\begin{array}{l}\text { ScaligD-F } \\
\text { ScaligD-R }\end{array}$ & $\begin{array}{l}\text { TCGTCGAAGGCACTAGAAGGG } \\
\text { CGGTCGATCTTGACGGCTG } \\
\text { GGTCGATCCCCGCATATAGGT } \\
\text { GCCGCCGGGCGTTTTTTAT }\end{array}$ & $\begin{array}{l}\text { ScaligD expression } \\
\text { cassette amplification }\end{array}$ \\
\hline $\begin{array}{l}38 \\
39\end{array}$ & $\begin{array}{l}\text { orf1-6 ligD test-F } \\
\text { orf1-6 ligD test-R }\end{array}$ & $\begin{array}{l}\text { CCGCCGACACCCCGATCACC } \\
\text { ACCGCAGCTTCCGCTCCCTG }\end{array}$ & $\begin{array}{l}\text { Check NHEJ for ac- } \\
\text { t/ORF1 editing }\end{array}$ \\
\hline $\begin{array}{l}40 \\
41\end{array}$ & $\begin{array}{l}\text { vb2 ligD test-F } \\
\text { vb2 ligD test- } R\end{array}$ & $\begin{array}{l}\text { CGAGGTGATCGACGCCAACC } \\
\text { TCGCCGAGCAGGATGATGTG }\end{array}$ & $\begin{array}{l}\text { Check NHEJ for } \\
\text { act } V B \text { editing }\end{array}$ \\
\hline
\end{tabular}


\#: The restriction sites are underlined; the $20 \mathrm{nt}$ target sequences are shown in bold, the pattern of the sgRNA-F primer is:

\section{CATGCCATGGN ${ }_{20}$ GTTTTAGAGCTAGAAATAGC.}

$5 \quad$ *: The overlap sequence for Gibson assembly is shown in italic.

$\S:$ The restriction sites are underlined.

Table 5. Strains and plasmids

\begin{tabular}{|c|c|c|}
\hline Name & Description & Reference \\
\hline WT & Streptomyces coelicolor A3(2) & $\begin{array}{l}95 \text { SNPs and } 1 \\
\text { deletions of (Bent- } \\
\text { ley et al., 2002) }\end{array}$ \\
\hline No Target & WT with pCRISPR-Cas9 & This study \\
\hline Mismatch & $\begin{array}{l}\text { WT with sgRNA: Actlorf1-1 NT including its } \\
\text { PAM sequence }\end{array}$ & This study \\
\hline$\Delta$ actlorf1-1 & $\begin{array}{l}\text { WT with pCRISPR-Cas9 carrying sgRNA: Ac- } \\
\text { tlorf1-1 NT, } 1 \text { bp insertions from the DSB site }\end{array}$ & This study \\
\hline$\Delta$ actlorf1-2 & $\begin{array}{l}\text { WT with pCRISPR-Cas } 9 \text { carrying sgRNA: Ac- } \\
\text { tlorf1-6 T, } 10721 \text { bp deletion around the DSB } \\
\text { site }\end{array}$ & This study \\
\hline$\Delta a c t v b-1$ & $\begin{array}{l}\text { WT with pCRISPR-Cas9 carrying sgRNA: } \\
\text { Actvb-2 NT, } 14716 \text { bp deletion around the DSB } \\
\text { site }\end{array}$ & This study \\
\hline$\Delta a c t v b-2$ & $\begin{array}{l}\text { WT with pCRISPR-Cas9 carrying sgRNA: } \\
\text { Actvb-5 NT, } 37173 \text { bp deletion around the DSB } \\
\text { site }\end{array}$ & This study \\
\hline $\begin{array}{l}\Delta \text { actlorf1- } \\
\text { ligD1- } \\
\Delta \text { actlorf1-ligD8 }\end{array}$ & $\begin{array}{l}\text { WT with pCRISPR-Cas9-ScaligD carrying sgR- } \\
\text { NA: Actlorf1-6 T, } 8 \text { random red clones }\end{array}$ & This study \\
\hline $\begin{array}{l}\Delta a c t v b-l i g D 1- \\
\Delta a c t v b-l i g D 8\end{array}$ & $\begin{array}{l}\text { WT with pCRISPR-Cas9-ScaligD carrying sgR- } \\
\text { NA: Actvb-2 NT, } 8 \text { random red clones }\end{array}$ & This study \\
\hline $\begin{array}{l}\text { orf1 deletion1- } \\
\text { orf1 deletion10 }\end{array}$ & $\begin{array}{l}\text { WT with actIORF1 recombination arm in the } \\
\text { pCRISPR-Cas } 9 \text { carrying sgRNA: Actlorf1- } 6 \text { T, } \\
\text { actIORF1 gene was deleted, } 10 \text { random clones }\end{array}$ & This study \\
\hline
\end{tabular}




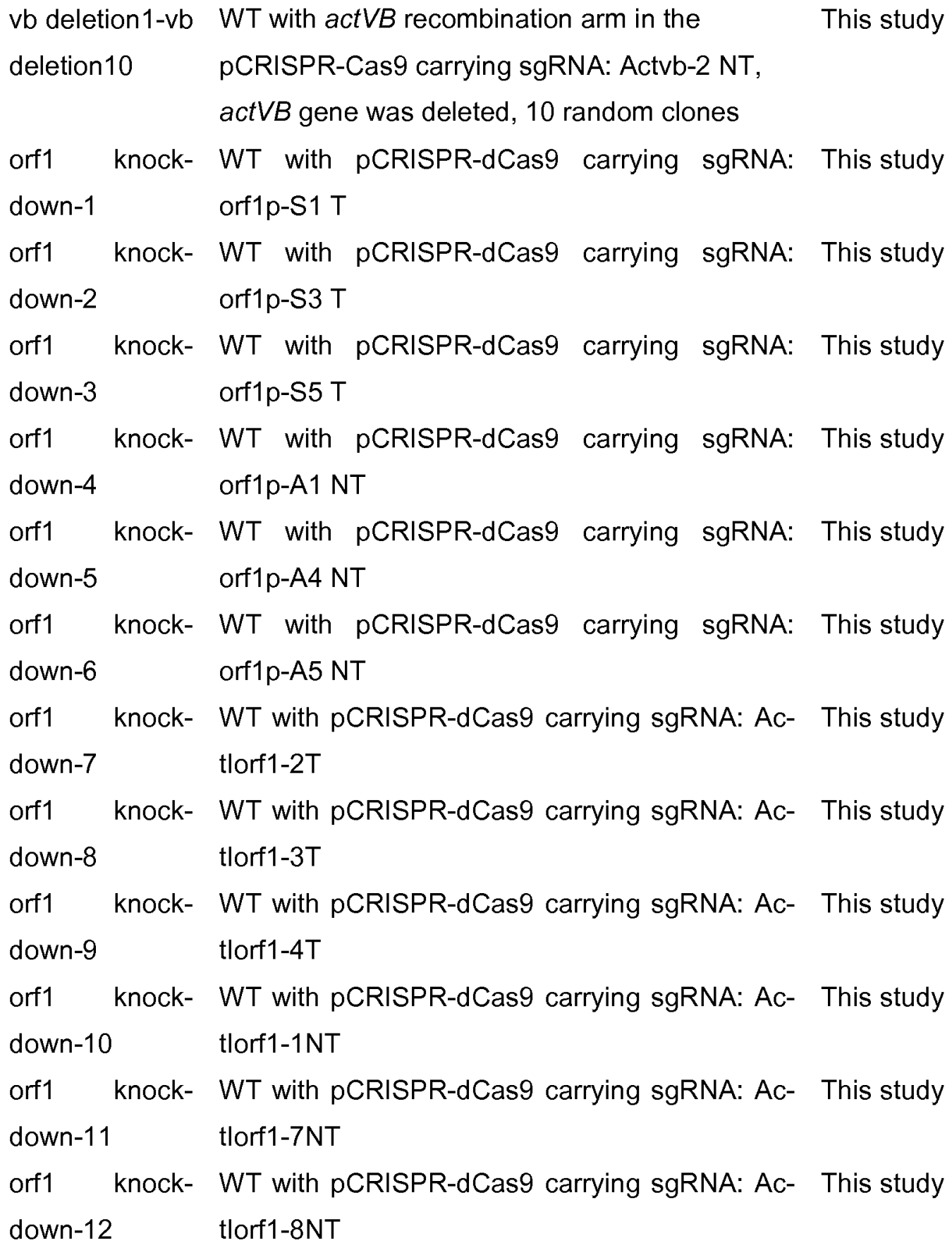


sgRNA

pCRISPR- $\quad$ pGM1190-sgRNA with cas9

This study

Cas9

pCRISPR- pGM1190-sgRNA with dcas9 (D10A and This study dCas9 H840A)

pCRISPR- $\quad$ pCRISPR-Cas9 with a ScaligD expression cas- This study

Cas9-ScaligD sette

pCRISPR- $\quad$ pCRISPR-Cas9 carrying sgRNA: Actlorf1-1 NT This study

Cas9-orf1-1

pCRISPR- $\quad$ pCRISPR-Cas9 carrying sgRNA: Actlorf1-2 T This study

Cas9-orf1-2

pCRISPR- $\quad$ pCRISPR-Cas9 carrying sgRNA: Actlorf1-3 T This study

Cas9-orf1-3

pCRISPR- $\quad$ pCRISPR-Cas9 carrying sgRNA: Actlorf1-4 T This study

Cas9-orf1-4

pCRISPR- $\quad$ pCRISPR-Cas9 carrying sgRNA: Actlorf1-5 T This study

Cas9-orf1-5

pCRISPR- $\quad$ pCRISPR-Cas9 carrying sgRNA: Actlorf1-6 T This study

Cas9-orf1-6

pCRISPR- $\quad$ pCRISPR-Cas9 carrying sgRNA: Actvb-1 NT This study

Cas9-vb1

pCRISPR- $\quad$ pCRISPR-Cas9 carrying sgRNA: Actvb-2 NT This study

Cas9-vb2

pCRISPR- $\quad$ pCRISPR-Cas9 carrying sgRNA: Actvb-3 T This study

Cas9-vb3

pCRISPR- $\quad$ pCRISPR-Cas9 carrying sgRNA: Actvb-4 T This study

Cas9-vb4

pCRISPR- $\quad$ pCRISPR-Cas9 carrying sgRNA: Actvb-5 NT This study

Cas9-vb5

pCRISPR- $\quad$ pCRISPR-Cas9 carrying sgRNA: Actvb-6 NT This study

Cas9-vb6

pCRISPR- $\quad$ pCRISPR-Cas9-orf1-6 with actIORF1 homolo- This study

Cas9-orf1-6- gous recombination template

Tem

pCRISPR- pCRISPR-Cas9-vb2 with actVB homologous This study 


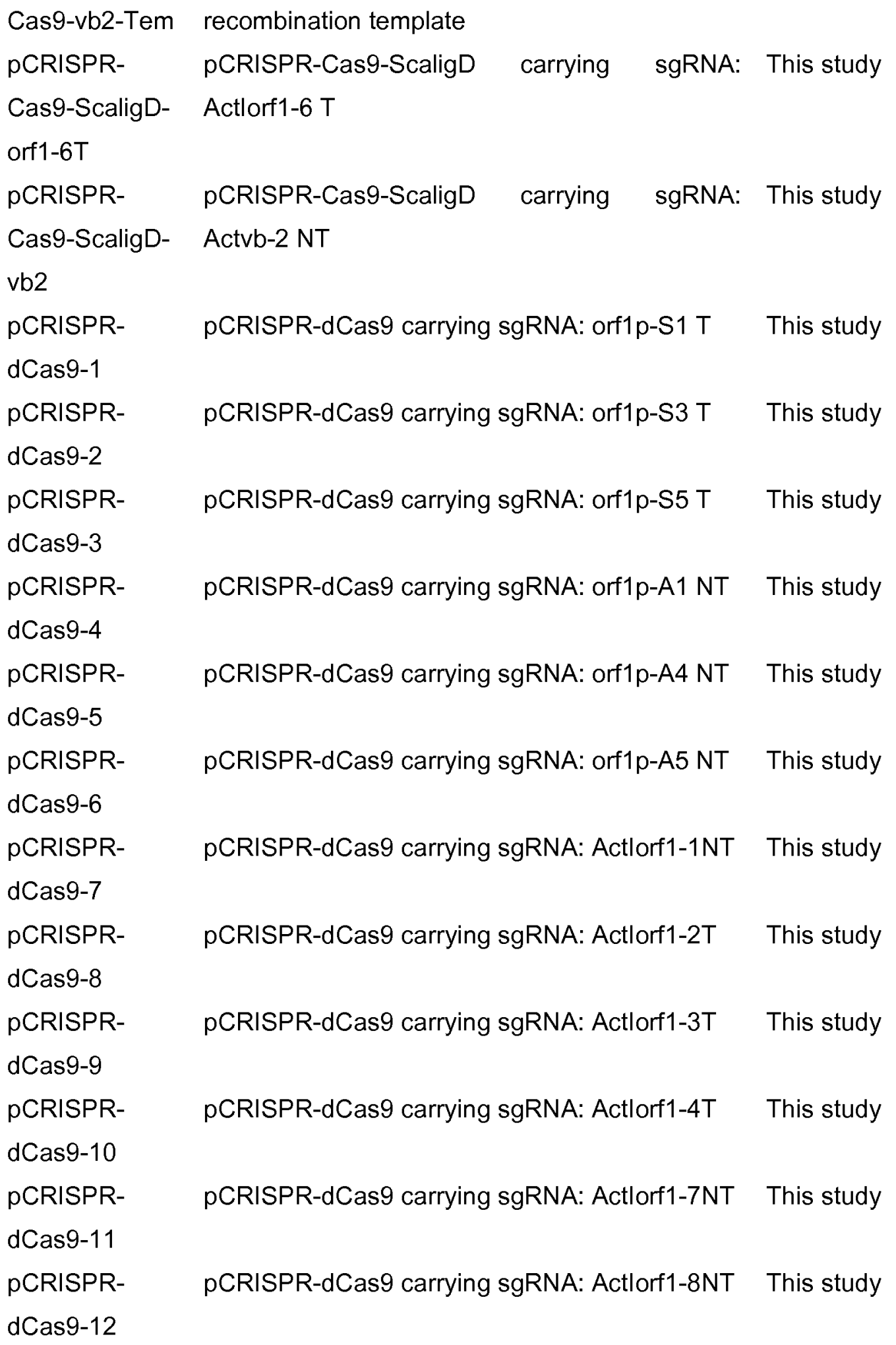


Cas 9 codon optimization for streptomycetes

The most studied CRISPR-Cas9 system is from Streptococcus pyogenes. As there is significant difference of GC content (35\% vs. $72 \%)$ and codon usage between S. pyogenes and Streptomyces coelicolor, a codon optimization of the S. pyogenes cas 9 according to the codon usage of streptomycetes was performed. In order to make the optimized cas 9 as compatible as possible for all streptomycetes, the codon usage table of the most studied actinomycete, Streptomyces coelicolor was used as template for codon optimization, using the $S$. pyogenes cas9 sequence as starting sequence (SEQ ID NO: 3$)$.

The codon optimization was done by GenScript inc. using the OptimumGeneTM algorithm, which optimizes a variety of parameters critical to the efficiency of gene expression, including but not limited to: codon usage bias, GC content, CpG dinucleotides content, mRNA secondary structure, cryptic splicing sites, premature PolyA sites, internal chi sites and ribosomal binding sites, negative $\mathrm{CpG}$ islands, RNA instability motif (ARE), repeat sequences (direct repeat, reverse repeat, and Dyad repeat) and restriction sites that may interfere with cloning.

The S. pyogenes cas 9 gene comprises tandem rare codons that can reduce the efficiency of translation or even disengage the translational machinery. The codon usage bias in Streptomyces coelicolor was modified by upgrading the CAI from 0.09 to 0.94 . GC content (from 35.04 to 61.79 ) and unfavorable peaks were optimized to prolong the half-life of the mRNA. The Stem-Loop structures, which impact ribosomal binding and stability of mRNA, were broken. In addition, negative cis-acting sites were screened and successfully modified.

\section{Design of the sgRNA scaffold}

The sequence of the core guide RNA is GTTTTAGAGCTAGAAATAGCAAGTTAAAATAAGGCTAGTCCGTTATCAACTTGAAAAAGTGGCACCGAGTCGGTGCTTTTTT (SEQ ID NO: 67); the RNA structure is shown in Figure 1. An ermE* promoter was introduced upstream the core sequence and two unique restriction sites, $\mathrm{Ncol}$ and $\mathrm{SnaBI}$ (underlined) were introduced into the scaffoled in order to make the scaffold easy adaptable when changing the $20 \mathrm{nt}$ target sequences. When constructing new functional sgRNAs, only the $20 \mathrm{nt}$ target sequence of the forward primer needs be changed, while the reverse primer including the $S$ naBI restriction site needs not be changed. 
The fragment is amplified by PCR and digested using the Ncol and SnaBI sites before cloning the functional sgRNA into the vector, under the control of the ermE* promotor (Figure 2). The final sgRNA scaffold sequence is: GCGGTCCACACGTGGCACCGCGATGCTGTTGTGGGCACAATCGTGCCGGTTGGTAGGATCGAC-

\section{GGCCATGG( $\left.\mathbf{N}_{20}\right)$ GTTTTAGAGCTAGAAATAGCAAGTTAAAATAAGGCTAGTCCGTTA} TCAACTTGAAAAAGTGGCACCGAGTCGGTGCTTTTTTTACGTA (SEQ ID NO: 68), where $\mathrm{N}_{20}$ represents the $20 \mathrm{nt}$ target sequence.

For the "one plasmid strategy", we selected the vector pGM1190 (Muth et al., 1989) as the backbone. pGM1190 is temperature sensitive in streptomycetes and will be lost at temperatures above $34^{\circ} \mathrm{C}$; the selection markers are apramycin and thiostrepton, the regulatory elements include: a thiostrepton-inducible promoter tipA, a RBS, a to and an $f d$ terminator. This plasmid can be shuttled in E. coli and streptomycetes.

The sgRNA scaffold was subcloned into pGM1190 upstream of the to terminator using the Gibson cloning method, resulting in pGM1190-sgRNA. The to terminator exited in pGM1190 is used as a secondary terminator for the sgRNA scaffold. Alternatively, it can be sub-cloned into a different vector; this strategy is termed the 'two plasmids strategy'.

\section{Construction of one plasmid based CRISPR-Cas9 system}

25 The codon optimized Cas9 was synthetized as set forth in SEQ ID NO: 1, flanked by the following restriction sites: CATATG in the 5'-end, where ATG is the start codon of SEQ ID NO: 1; and AAGCTTTCTAGA in the 3'-end, immediately downstream of the stop codon.

30 For the one plasmid strategy, the gene was sub-cloned into pGM1190-sgRNA with Ndel and Xbal sites, under the control of the thiostrepton inducible tipA promoter. The final vector was named $p$ CRISPR-Cas 9 (Figure 3 ). The sgRNA and cas 9 fragments were confirmed by PCR (with the primers, sgRNA check-F and sgRNA check-R) and digested by Ndel and Xbal. 
Insertion of the target sequence into the guide RNA

In order to construct a functional vector for the one plasmid strategy, it is sufficient to introduce the $20 \mathrm{nt}$ target sequence upstream of the sgRNA. Design software such as CRISPRy and other similar software can be used for sgRNA design. Here, we used CRISPRy for S. coelicolor (http://staff.biosustain.dtu.dk/laeb/crispy_scoeli/ or , or http://crispy.secondarymetabolites.org).

Based on the specificity of the target sequences with the gene, one or more target sequences were chosen. Based on the target sequences, the forward PCR primer as designed: CATGCCATGG $\mathrm{N}_{20}$ GTTTTAGAGCTAGAAATAGC $\left(\mathrm{N}_{20}\right.$ is the $20 \mathrm{nt}$ target sequence) (SEQ ID NO: 69), while the reverse primer remains the same: ACGCCTACGTAAAAAAAGCACCGACTCGGTGCC (sgRNA-R; SEQ ID NO: 44) (the restriction sites are underlined). PCR as used to amplify the functional sgRNAs from the pCRISPR-Cas9 template. The PCR products were digested with Ncol and SnaBI. The pCRISPR-Cas9 was also digested with the same restriction enzymes. After agrose gel purification, the $\sim 110 \mathrm{bp} \mathrm{PCR}$ fragment and the $\sim 11 \mathrm{~kb}$ pCRISPR-Cas9 backbone were ligated by $\mathrm{T} 4$ ligase and the ligation mix was transformed into competent $E$. coli. Several positive transformants for each target sequence were picked for colony PCR screening using the primers, sgRNA check-F and sgRNA check-R. The expected sizes were 234 bp for positive clones and were confirmed by sequencing.

\section{Example 2: generation of random-sized deletions around a target site}

This example describes how to apply the present method to inactivate the actinorhdin biosynthetic genes, as well as control the target gene expression in Streptomyces coelicolor A3(2). S. coelicolor A3(2) is a well-known actinorhdin producer. Actinorhodin is a benzoisochromanequinone polyketide antibiotic with $\mathrm{pH}$-dependent colors: blue color when $\mathrm{pH}>7$, red color when $\mathrm{pH}<7$.

Actinorhdin biosynthesis is encoded by a PKS type II gene cluster, named act gene cluster (Figure 4). The steps to synthetize actinorhodin are: I. 1X Acetyl-CoA and 7x malonyl-CoA are condensed to form the carbon skeleton by Actl; II. The above carbon backbone is cyclized to form a three ring intermediate, DNPA by ActIII, ActVII, ActIV, ActVI-1 and ActVI-3; III. DNPA is then modified to form DHK by ActVI-2, ActVI-4 and ActVA-6; IV. 2 DHK is dimerized to form the final product, actinorhodin, by ActVA-5 and ActVB (Figure 4). Two genes were selected as targets (marked by arrows in Figure 4): 
ActORF1 is the actinorhodin ketosynthase subunit alpha (KS domain of PKS II), and ActVB is the actinorhodin polyketide dimerase. A deletion of any of these two genes results in a loss of actinorhodin production, which can be easily monitored by the disappearance of the blue pigment.

For each gene inactivation, 6 different sgRNAs were designed for each gene using CRISPRy webserver (http://staff.biosustain.dtu.dk/laeb/crispy scoeli/), resulting in 12 sgRNAs (listed in Table 3).

10

PCR was used to amplify the functional sgRNAs from the pCRISPR-Cas9 template (for primers, see Table 4). The fragments and pCRISPR-Cas9 were digested using Ncol and SnaBI. After agarose gel purification, the PCR fragment (1 10 bp) and the pCRISPR-Cas9 backbone $(\sim 11 \mathrm{~kb})$ were ligated, and transferred into One Shot ${ }^{\circledast}$

15 Mach $1^{\mathrm{TM}}-\mathrm{T} 1^{\mathrm{R}}$ chemically competent $E$. coli. 6 positive transformants for each target sequence were picked for colony PCR screening using the primers set, sgRNA check$\mathrm{F}$ and sgRNA check-R (Table 4), a set of primers resulting in products of $234 \mathrm{bp}$ for positive clones and $214 \mathrm{bp}$ for the negative clones. The PCR screening results are shown in Figure 10A-F (A-C for actIORF1, D-F for actVB).

2-3 positive clones for each target sequence were confirmed by sequencing and matched the results of the colony PCR $100 \%$. Colony PCR is thus a valid way of screening the clones.

25 One correct clone for each target sequences was selected randomly to be transferred into the ET12567/pUZ8002 E. coli strain for conjugation. In addition, two negative controls were used: the first is the empty vector, pCRISPR-Cas9 (No Target), which has no target matches on the genome, and the second is a target sequence with a $3 \mathrm{nt}$ PAM motif "NGG". The inclusion of the PAM as part of the sgRNA abolishes correct recognition of the genomic target (Mismatch).

The PCR validated conjugates for each target sequence plus the two controls were inoculated into $20 \mathrm{ml} \mathrm{LB}$ broth with $25 \mu \mathrm{g} / \mathrm{ml}$ kanamycin, $25 \mu \mathrm{g} / \mathrm{ml}$ chloramphenicol and $50 \mu \mathrm{g} / \mathrm{ml}$ apramycin. After overnight shaking at $37^{\circ} \mathrm{C}$, the $E$. coli cells were harvested by centrifuging at $5000 \mathrm{~g}$ for 5 minutes at room temperature; fresh LB was used without 
antibiotics to wash 2 times. The donor cells then were resuspended in $0.5-2 \mathrm{ml} L B$ broth and placed at room temperature. To collect S. coelicolor, spores from one ISP2 plate were resuspended in $0.9 \%$ saline, and filtered through a cotton pad. The spore suspension was concentrated by centrifuging at $5000 \mathrm{~g}$ for 5 minutes at room temperature, then the spores were resuspended in $0.5 \mathrm{ml}-1 \mathrm{ml} 2 \times Y \mathrm{~T}$ broth. To induce germination, the spore suspension was heated to $50^{\circ} \mathrm{C}$ for 10 minutes, and then cooled down to room temperature. $500 \mu$ l of the relevant ET12567/pUZ8002 cells were added to the heat treated pre-germinated spores and mixed by inversion. The mixture was centrifuged for 2 minutes at top speed, the supernatant was decanted and the pellet was resuspended in the remaining fluid so that the final volume was about $50 \mu \mathrm{l}$. The cells were then plated on Cullum agar plates and incubated for $16 \mathrm{~h}$ at $30^{\circ} \mathrm{C}$. After $16 \mathrm{~h}$, the plates were overlaid with a solution containing the selection antibiotics: $20 \mu \mathrm{l}$ of 50 $\mathrm{mg} / \mathrm{ml}$ nalidixic acid, against $E$. coli cells or $10 \mu \mathrm{l}$ of $100 \mathrm{mg} / \mathrm{ml}$ apramycin for the selection of clones with the transferred DNA, dissolved in $1 \mathrm{ml}$ of sterile $\mathrm{H}_{2} \mathrm{O}$. The overlaid plates were further incubated for $3-7$ days at $30^{\circ} \mathrm{C}$, or until colonies became visible. 50 80 conjugates for each target sequence were randomly picked onto ISP2 plates with 50 $\mu \mathrm{g} / \mathrm{ml}$ apramycin, $50 \mu \mathrm{g} / \mathrm{ml}$ nalidixic acid (to avoid $E$. coli contamination), and $1 \mu \mathrm{g} / \mathrm{ml}$ thiostrepton (to induce Cas9). In parallel, the same sets of clones were also streaked onto ISP2 plate with $50 \mu \mathrm{g} / \mathrm{ml}$ apramycin and $50 \mu \mathrm{g} / \mathrm{ml}$ nalidixic acid, but without thiostrepton. The plates were incubated for $7-10$ days at $30^{\circ} \mathrm{C}$.

From the red colonies, the following clones were randomly selected: one clone for each gene ( $\Delta a c t l o r f 1-1$ and $\Delta a c t v b-1$ ), as well as one clone for each negative control (Mismatch and No Target), and one clone for the wild type (WT), resulting in 5 strains (Figure 6 and Figure 7).

Besides ISP2 agar plates, the above selected five strains (from ISP2 plates with thiostrepton) were also inoculated in $100 \mathrm{ml}$ ISP2 liquid medium, and incubated with shaking for 7 days at $30^{\circ} \mathrm{C} .30 \mathrm{ml}$ cultures were used for each strain to perform actinorhodin extraction. The cultures were centrifuged at $8000 \mathrm{~g}$ for 10 minutes at room temperature, the supernatant was transferred to a $50 \mathrm{ml}$ tube, the $\mathrm{pH}$ was adjusted to 2 with $1 \mathrm{M}$ $\mathrm{HCl}$, before adding $1 / 4$ volume chloroform. The solution was intensively mixed by vortex, and then centrifuged at $8000 \mathrm{~g}$ for 5 minutes at room temperature. The chloroform phase was collected for drying, the dried samples were re-dissolved using $2 \mathrm{ml}$ solvent (methanol: chloroform=1:1). The solutions were analyzed using the Evolution ${ }^{\mathrm{TM}}$ 
201/220 UV-Visible Spectrophotometers to scan from $420 \mathrm{~nm}$ to $720 \mathrm{~nm}$ (the actinorhodin in these conditions has a maximum absorption at about $530 \mathrm{~nm}$ ). The scanning results show that the actinorhodin peaks in $\Delta a c t / o r f 1-1$ and $\Delta a c t v b-1$ disappeared (Figure 7).

Genomic DNA was extracted using $10 \mathrm{ml}$ of the above cultures for each strain using Blood \& Cell Culture DNA Kit (QIAGEN, Germany). The genomic libraries were generated using the TruSeq $®$ Nano DNA LT Sample Preparation Kit (Illumina Inc., San Diego CA). Briefly, $100 \mathrm{ng}$ of genomic DNA diluted in $52.5 \mu \mathrm{l}$ TE buffer was fragmented in Covaris Crimp Cap microtubes on a Covaris E220 ultrasonicator (Covaris, Brighton, UK) with $5 \%$ duty factor, $175 \mathrm{~W}$ peak incident power, 200 cycles/burst, and $50 \mathrm{~s}$ duration under frequency sweeping mode at 5.5 to $6^{\circ} \mathrm{C}$ (Illumina recommendations for a 350-bp average fragment size). The ends of fragmented DNA were repaired by T4 DNA polymerase, Klenow DNA polymerase, and T4 polynucleotide kinase. The Klenow exo minus enzyme was then used to add an ' $A$ ' base to the 3 ' end of the DNA fragments. After the ligation of the adapters to the ends of the DNA fragments, DNA fragments ranging from $300-400$ bp were recovered by bead purification. Finally, the adapter-modified DNA fragments were enriched by 3 cycle-PCR. The final concentration of each library was measured by Qubit $₫$ 2.0 Florometer and Qubit DNA Broad range assay (Life Technologies, Paisley, UK). The average sizes of the dsDNA libraries were determined using the Agilent DNA 7500 kit on an Agilent 2100 Bioanalyzer. Libraries were normalised and pooled in $10 \mathrm{mM}$ Tris- $\mathrm{Cl}, \mathrm{pH} 8.0$, plus $0.05 \%$ Tween 20 to the final concentration of $10 \mathrm{nM}$. After denaturation in $0.2 \mathrm{~N} \mathrm{NaOH}$, a $10 \mathrm{pm}$ pool of 20 libraries in $600 \mu \mathrm{l}$ ice-cold HT1 buffer was loaded onto the flow cell provided in the MiSeq Reagent kit v2 (300 cycles) and sequenced on a MiSeq (Illumina Inc., San Diego, CA) platform with a paired-end protocol and read lengths of $151 \mathrm{nt}$.

Mapping of the sequencing reads to the S. coelicolor A3(2) reference genome (Genbank accession AL645882).

30 The reads obtained above were mapped to the $S$ coelicolor $A 3(2)$ reference genome using the software BWA (Li et al., 2009) using the BWA-mem algorithm. The data was inspected and visualized using readXplorer (Hilker et al., 2014) and Artemis (Rutherford et al., 2000). Comparison of the refererence S. coelicolor A3(2) wild type strain used in this study with the $S$. coelicolor $A 3(2)$ reference sequence deposited as AL645882 in Genbank resulted in 95 SNPs and fragment (5797650-5818686) dele- 
tion. For the following, S. coelicolor A3(2) WT refers to the sequences obtained in this study. The detailed mapping results are shown Table 6.

Table 6. List of mutations detected from whole genome sequencing (the results 5 shown are after subtracted from the WT)

\begin{tabular}{|c|c|c|c|c|c|}
\hline Name & Position & Mutation & Annotation & Gene & Description \\
\hline \multirow[t]{5}{*}{ Mismatch } & $2,474,084$ & $A \rightarrow C$ & $\begin{array}{l}\text { T8P } \\
(\mathrm{ACC} \rightarrow \mathrm{CC} \\
\mathrm{C})\end{array}$ & $\mathrm{SCO} 2305 \rightarrow$ & $\begin{array}{l}\text { putative ABC } \\
\text { transporter } \\
\text { ATP-binding sub- } \\
\text { unit }\end{array}$ \\
\hline & $4,477,934$ & $2 \mathrm{bp} \rightarrow \mathrm{TC}$ & $\begin{array}{l}\text { coding } \\
(195- \\
196 / 609 \mathrm{nt})\end{array}$ & SCO4084 $\rightarrow$ & $\begin{array}{l}\text { hypothetical pro- } \\
\text { tein SCD } 25.20\end{array}$ \\
\hline & $8,265,166$ & $\mathrm{G} \rightarrow \mathrm{C}$ & $\begin{array}{l}\text { intergenic } \\
(+76 /-125)\end{array}$ & $\begin{array}{l}\text { SCO7449 } \rightarrow I \\
\rightarrow \text { SCO7450 }\end{array}$ & $\begin{array}{l}\text { putative mem- } \\
\text { brane protein. } \\
\text { /putative secreted } \\
\text { protein }\end{array}$ \\
\hline & $8,267,257$ & $\mathrm{G} \rightarrow \mathrm{C}$ & $\begin{array}{l}\text { intergenic } \\
(+13 /+26)\end{array}$ & $\begin{array}{l}\text { SCO7451 } \rightarrow / \\
\leftarrow \text { SCO7452 }\end{array}$ & $\begin{array}{l}\text { conserved hypo- } \\
\text { thetical protein } \\
\text { SC } 5 \mathrm{C} 11.08 / \text { putati }\end{array}$ \\
\hline & & & & & $\begin{array}{l}\text { ve } \\
\text { methyltransfer- } \\
\text { ase. }\end{array}$ \\
\hline \multirow[t]{2}{*}{$\begin{array}{l}\text { No Tar- } \\
\text { get }\end{array}$} & $1,645,577$ & $+G$ & $\begin{array}{l}\text { intergenic (- } \\
554 /+422)\end{array}$ & $\begin{array}{l}\text { SCO1536 I } \\
\leftarrow S C 01537\end{array}$ & $\begin{array}{l}\text { conserved hypo- } \\
\text { thetical protein } \\
\text { SCL2.26c/putativ } \\
\text { e transport sys- } \\
\text { tem membrane } \\
\text { protein }\end{array}$ \\
\hline & $1,645,634$ & $A \rightarrow G$ & $\begin{array}{l}\text { intergenic (- } \\
611 /+365)\end{array}$ & $\begin{array}{l}\text { SCO1536 I } \\
\longleftarrow \text { SC01537 }\end{array}$ & $\begin{array}{l}\text { conserved hypo- } \\
\text { thetical protein } \\
\text { SCL2.26c/putativ } \\
\text { e transport sys- } \\
\text { tem membrane } \\
\text { protein }\end{array}$ \\
\hline
\end{tabular}


$2,462,898 \quad(\mathrm{G}) 12 \rightarrow 13 \quad$ intergenic $(-\mathrm{SCO} 2292 \longleftarrow \mathrm{l}$ secreted endo386/+324) $\leftarrow \mathrm{SCO} 2293$ 1,4-beta-xylanase

B (xylanase

B)/putative integral membrane protein

$5,093,984 \quad G \rightarrow C \quad$ P550A $\quad$ SCO4664 $\leftarrow$ putative integral $(\mathrm{CCC} \rightarrow \mathrm{GC}$ membrane proC) tein

$6,442,710 \quad(G) 9 \rightarrow 10 \quad$ intergenic $(-\quad S C 05885 \leftarrow /$ putative 96/+43) «SCO5886 membrane protein/3-oxoacyl[acyl-carrierprotein] synthase II

8,163,408 $\mathrm{T} \rightarrow \mathrm{C} \quad \mathrm{T} 129 \mathrm{~T} \quad \mathrm{SCO} 3350 \leftarrow$ putative mem$(\mathrm{ACA} \rightarrow \mathrm{AC} \quad$ brane efflux pro-

G) tein.

$2,311,509 \quad(T G A) 4 \rightarrow$ coding $\quad$ SCO2148 $\leftarrow$ cytochrome $\quad B$ $5 \quad(176 / 1638 \quad$ subunit

nt)

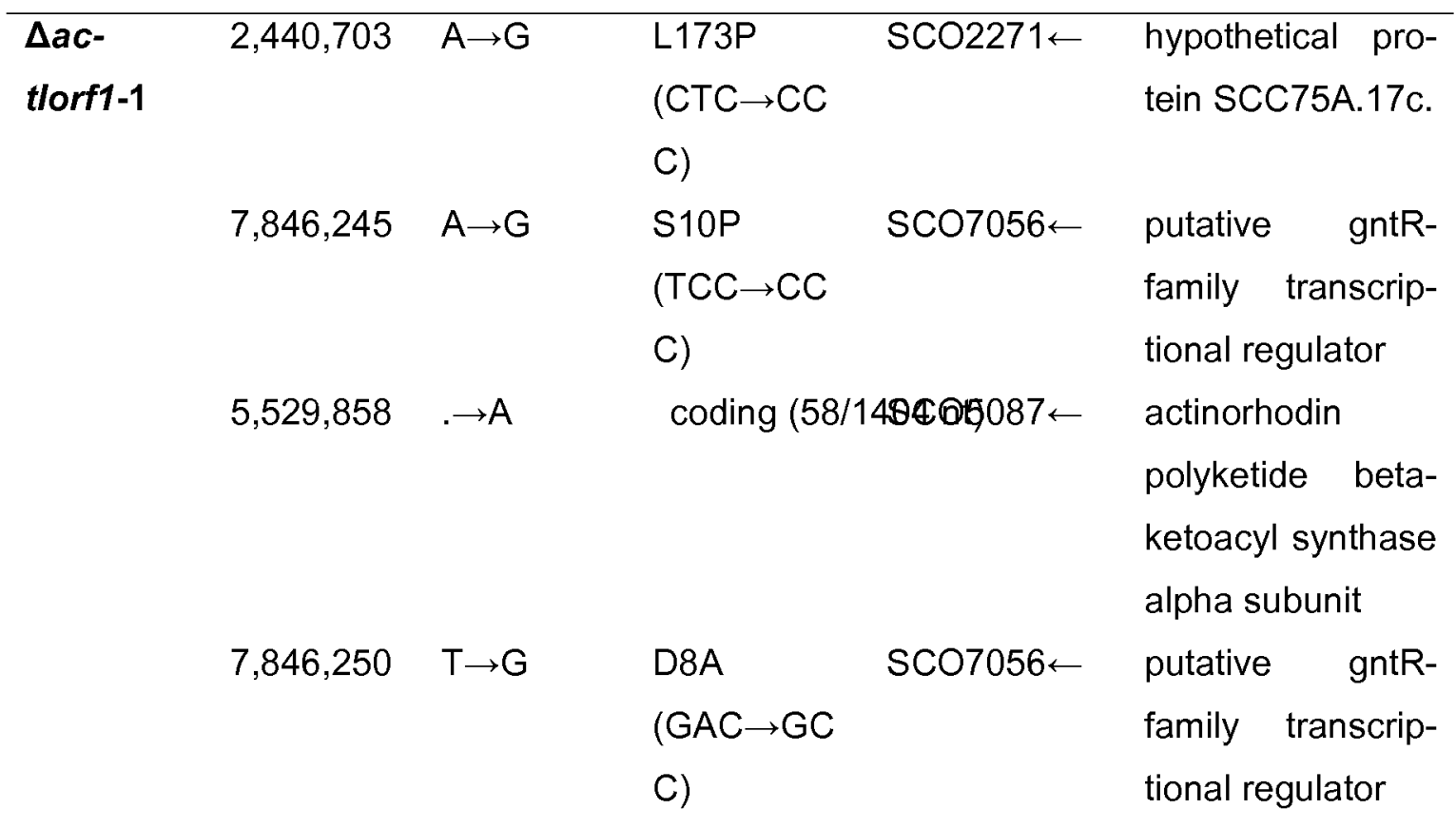

$\begin{array}{lllll}\Delta a c- & 2,462,898 & (\mathrm{G}) 12 \rightarrow 11 & \text { intergenic }(- & \text { SCO2292 } / \text { secreted endo- }\end{array}$ 


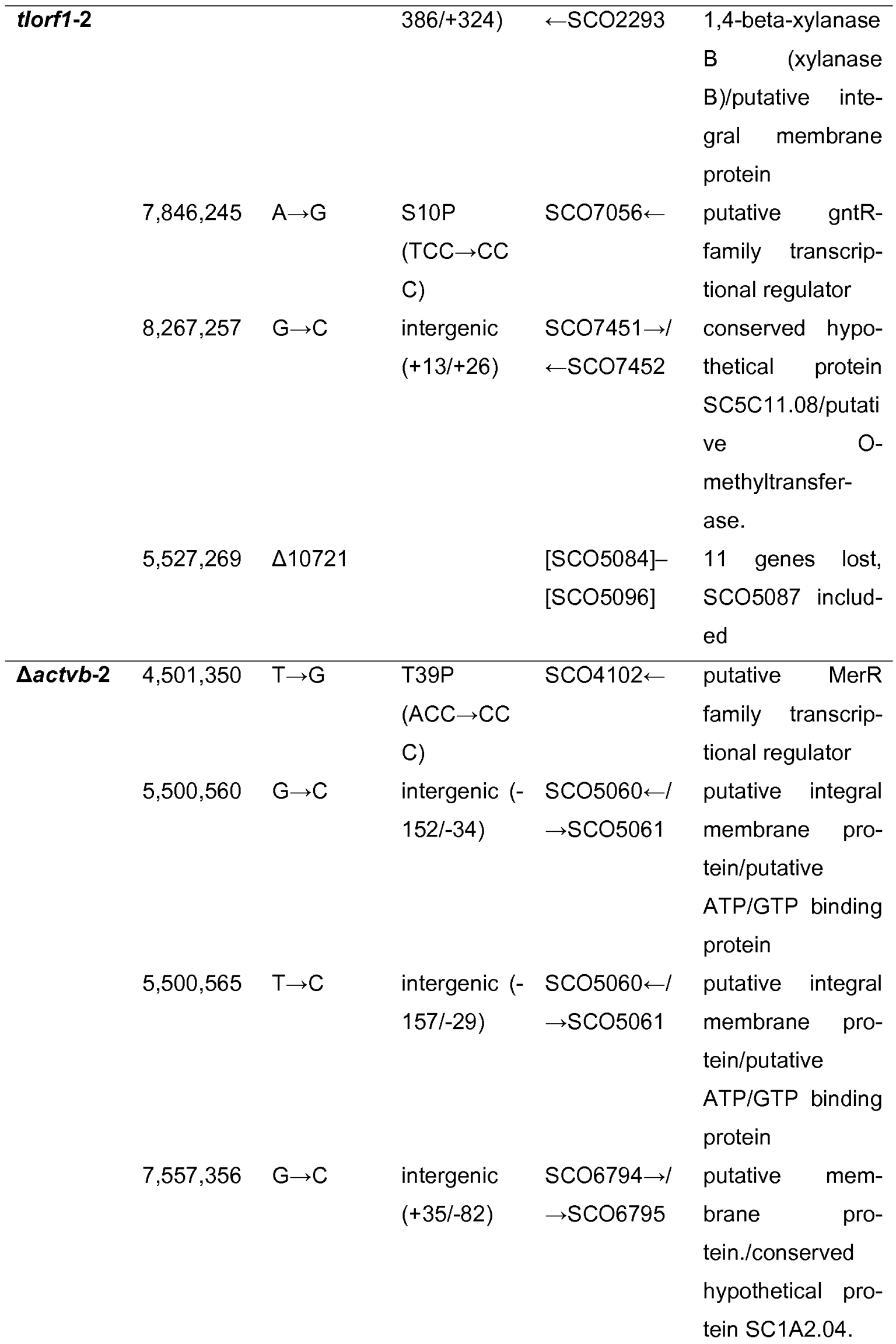




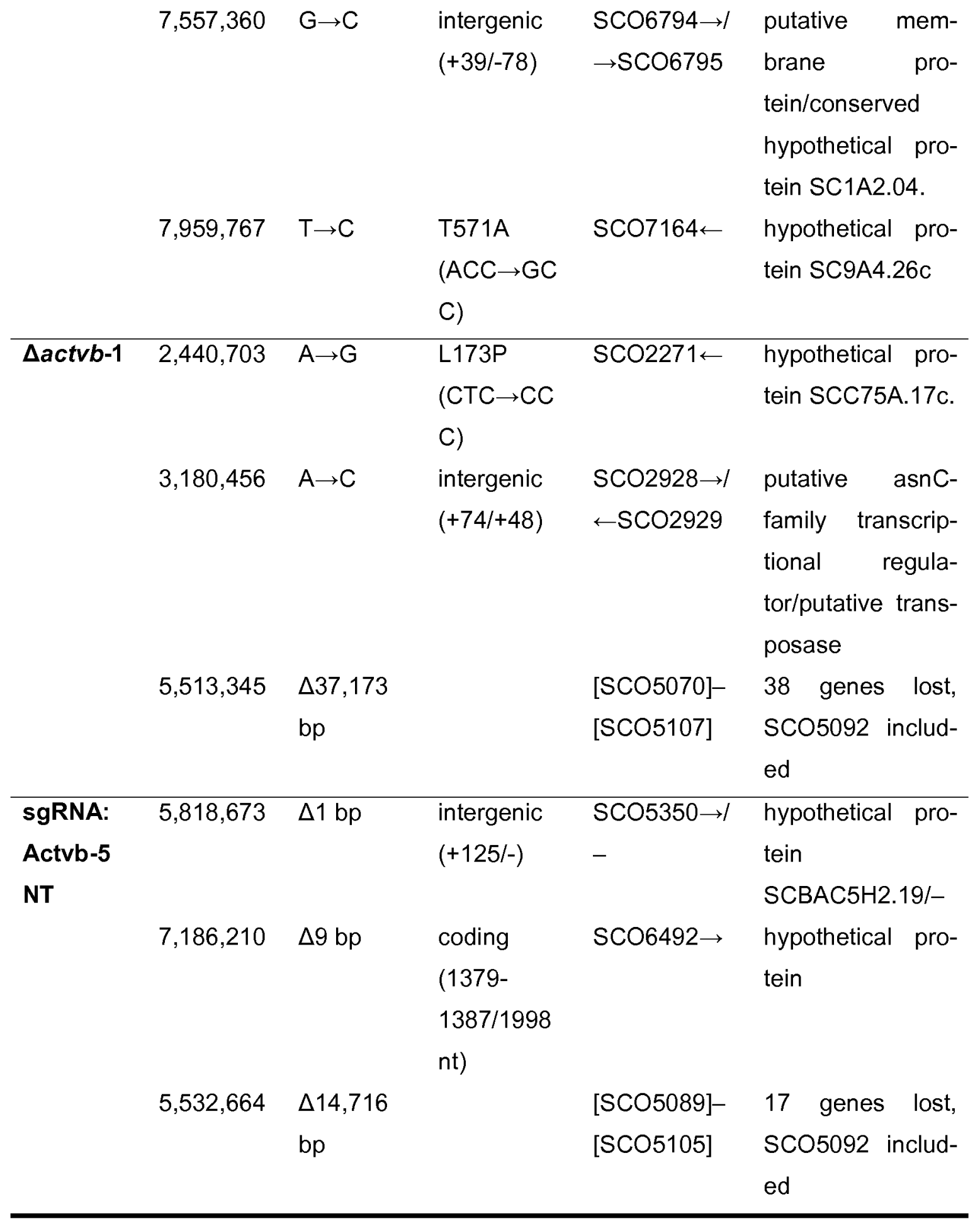

Interestingly, the inactivation of the genes were caused by rearrangement events including $1 \mathrm{bp}$ insertions and deletions between $1 \mathrm{bp}$ and more than $30000 \mathrm{bps}$ around 5 the DSB site (Figure 8A and B). In other words, the deletion can be both very precise 
and random sized around the DSB site. It appears this is effect is due to partially deficient NHEJ in S. coelicolor.

It was also tested whether deletions could be generated in other organisms. Deletions were successfully generated in Streptomyces collinus Tü365, in Streptomyces avermitilis, Streptomyces pristinaespiralis and Verrucosispora spp.

Streptomyces collinus Tü365 and in Verrucosispora spp. were investigated further , and random-sized deletions ranging from a few kilobase pairs to more than $1 \mathrm{~kb}$ were observed.

\section{Species tested}

Streptomyces collinus Tü365

Verrucosispora spp.

\section{Deletion size $(\mathrm{kb}) \quad$ Numbers of tested genes (gene clusters)}

23-1200 6

$5-80 \quad 3$

This example shows that the present method can be used to obtain a set of random sized deletions around a precisely defined site from a target sequence in different microorganisms using the present CRISPR-Cas9 system.

\section{Example 3: generation of precise deletions around a target site by introduction of a} functional NHEJ pathway

20 Genome mining indicated that the NHEJ pathway of some streptomycetes is not complete because one core component called DNA ligase D is missing. In order to reconstitute the NHEJ pathway of S. coelicolor, homologues of ligD were identified by blasting, using the mycobacterial lig $D$ amino acid sequence as a query. A homologue of ligD was found in S. carneus.

An S. carneus ligD expression cassette was designed, where the $S$. carneus ligD (ScaligD; SEQ ID NO: 70) was cloned under control of an ermE* promoter, and a to terminator introduced downstream of lig $D$. This expression cassette was subcloned into the Stul site of pCRISPR-Cas 9 by Gibson assembly. The construction was called pCRISPR-Cas9-ligD (Figure 9). 
One sgRNA was selected for each of the two targeted genes (sgRNA: Actlorf1-6 T for actlORF1, and sgRNA: Actvb-2 NT for actVB) to test whether the natively deficient NHEJ pathway was fixed.

5 Comparison to the non-ScaligD CRISPR-Cas9 system (example 2) showed that the inactivation efficiency increased from $45 \%$ to $77 \%$, and $37 \%$ to $69 \%$ for sgRNA: Actlorf1-6 T and sgRNA: Actvb-2 NT, respectively, after the ScaligD was introduced into the system (Table 7).

10

Table 7 The inactivation efficiency of different sgRNAs with different DSB repair pathways.

\begin{tabular}{|c|c|c|c|c|c|c|}
\hline \multirow{2}{*}{$\begin{array}{c}\text { Ways of } \\
\text { DSB re- } \\
\text { pair }\end{array}$} & \multirow{2}{*}{ sgRNAs } & \multicolumn{4}{|c|}{ Colony Count $^{a}$} & \multirow{2}{*}{$\begin{array}{c}\text { Efficiency (\%) } \\
\text { Red/Total }\end{array}$} \\
\hline & & No growth & $\operatorname{Red}^{b}$ & Blue & Total & \\
\hline \multirow{12}{*}{$\begin{array}{c}\text { Incom- } \\
\text { plete } \\
\text { NHEJ }\end{array}$} & $\begin{array}{l}\text { Actlorf1-1 } \\
\text { NT }\end{array}$ & 20 & 31 & 30 & 81 & 38 \\
\hline & $\begin{array}{l}\text { Actlorf1-2 } \\
T\end{array}$ & 3 & 1 & 7 & 11 & 9 \\
\hline & $\begin{array}{l}\text { Actlorf1-3 } \\
T\end{array}$ & 7 & 18 & 49 & 74 & 24 \\
\hline & $\begin{array}{l}\text { Actlorf1-4 } \\
T\end{array}$ & 43 & 10 & 1 & 54 & 19 \\
\hline & $\begin{array}{l}\text { Actlorf1-5 } \\
T\end{array}$ & 8 & 18 & 8 & 34 & 53 \\
\hline & $\begin{array}{l}\text { Actvb-1 } \\
\text { NT }\end{array}$ & 10 & 20 & 22 & 52 & 38 \\
\hline & Actvb-3 T & 17 & 6 & 40 & 63 & 10 \\
\hline & Actvb-4 T & 30 & 6 & 5 & 41 & 15 \\
\hline & $\begin{array}{l}\text { Actvb-5 } \\
\text { NT }\end{array}$ & 7 & 20 & 10 & 37 & 54 \\
\hline & $\begin{array}{l}\text { Actvb-6 } \\
\text { NT }\end{array}$ & 1 & 1 & 30 & 32 & 3 \\
\hline & $\begin{array}{l}\text { Actlorf1-6 } \\
T\end{array}$ & 10 & 18 & 12 & 40 & 45 \\
\hline & $\begin{array}{l}\text { Actvb-2 } \\
\text { NT }\end{array}$ & 20 & 13 & 2 & 35 & 37 \\
\hline \multirow{2}{*}{$\begin{array}{l}\text { Recons- } \\
\text { tituted } \\
\text { NHEJ }\end{array}$} & $\begin{array}{l}\text { Actlorf1-6 } \\
T\end{array}$ & 0 & 24 & 7 & 31 & 77 \\
\hline & $\begin{array}{l}\text { Actvb-2 } \\
\text { NT }\end{array}$ & 0 & 18 & 8 & 26 & 69 \\
\hline \multirow{2}{*}{$\begin{array}{c}\text { HDR (with } \\
\text { homology } \\
\text { tem- } \\
\text { plates) }\end{array}$} & $\begin{array}{l}\text { Actlorf1-6 } \\
T\end{array}$ & 0 & 52 & 0 & 52 & 100 \\
\hline & $\begin{array}{l}\text { Actvb-2 } \\
\text { NT }\end{array}$ & 0 & 35 & 1 & 36 & 97 \\
\hline
\end{tabular}


${ }^{a}$ Denotes the number of colonies with the indicated phenotype after induction with thiostrepton. ${ }^{b}$ Actinorhodin is blue. Upon loss of actinorhodin production, the red color of the $2^{\text {nd }}$ pigmented antibiotic, undecylprodigiosin, becomes visible.

To further validate this observation, primers were designed to detect the $\sim 600 \mathrm{bp}$ fragment containing the theoretical cleavage sites of the used sgRNAs. Eight red clones for each gene were randomly selected for colony PCR, and the PCR products were sequenced. No long fragment deletions were found in any of the 16 sequencing clones; instead, most of them just had 1 to 3 bp deletion, substitution, or insertion (Figure $8 \mathrm{C}$ and $\mathrm{D}$ ). In contrast, without the ScaligD, long fragment deletions were found in 3 of the 4 red clones for which whole genome sequencing was performed (Figure 8A).

These results indicated the natively deficient incomplete NHEJ pathway was successfully fixed by complementary its missing component, DNA ligase D.

\section{Example 4: HDR -directed gene editing}

In this example, in order to bypass the NHEJ pathway, a template for homologous recombination was introduced into the CRISPR-Cas9 system to let the organism use HDR to repair the DSBs. Again the genes ActIORF1 and ActVB were selected for testing, only one sgRNA (sgRNA: Actlorf1-6 T, and sgRNA: Actvb-2NT) was designed for each gene. PCR was used to amplify the $\sim 1 \mathrm{~kb}$ fragments of the 5' and the 3' regions out of the targeted genes with the primers orf1-5'F, orf1-5'R, orf1-3'F, orf1-3'R, and VB$5^{\circ} \mathrm{F}, \mathrm{VB}-5{ }^{\mathrm{A}} \mathrm{R}, \mathrm{VB}-3^{\mathrm{\prime}} \mathrm{F}, \mathrm{VB}-3^{\mathrm{c}} \mathrm{R}$, for actORF1 and actVB, respectively. The orf1-5'F and VB-5'F primers contain a 20 bp overlap region of the 5 ' of the Stul site from the pCRISPR-Cas9 plasmid, and the orf1-3'R and VB-3'R primers contain a 20 bp overlap region of the $3^{\prime}$ of the Stul site from the $p C R I S P R-C a s 9$ plasmid, while the orf $1-5^{\prime} R$ and VB-5'R primers contain a 20 bp overlap region of the orf1-3' fragment and VB-3' fragment, respectively. After gel purification of the fragments, orf1-5', orf1-3', and the Stul digested pCRISPR-Cas9 plasmid, and VB-5', VB-3', and the Stul digested pCRISPRCas9 plasmid were assembled by Gibson assembly (New England Biolabs). The transformants were screened by PCR using orf1-check-F, orf1-check-R and VB-check-F, VB-check-R for the homologous recombination templates of actIORF1 and actVB, respectively, and finally confirmed by sequencing. All 52 clones picked randomly for actIORF1, and 35 out of 36 clones picked randomly for actVB were red after induction (Table 7). 
In order to find out whether the deletion was a precise deletion, we designed primers around the target cleavage site. For both genes, 10 red clones were randomly selected for colony PCR validation. The colony PCR was performed as follows: mycelia of the selected colonies were scraped from the plates using a sterile toothpick into $10 \mu$ pure DMSO in PCR tubes. The tubes were shaken vigorously for $10 \mathrm{~min}$ at $100^{\circ} \mathrm{C}$ in a heating block. After this step, the solution was centrifuged at top speed for 10 seconds, $1 \mu \mathrm{l}$ of the supernatant were used for PCR template in a $20 \mu \mathrm{PCR}$ reaction.

10 The sizes of all 20 PCR products corresponded to the predicted sizes of the gene deletion (Figure 10). Importantly, the CRISPR-Cas9 system with the homologous recombination template showed even higher efficiency and precision in gene editing in comparison to the gene deletion system relying on functional NHEJ described in example 3 (Table 7).

This example shows that gene editing can be performed in actinomycetes using the CRISPR/Cas9 system with homologous recombination with high precision and efficiency.

\section{Example 5: modulation of gene expression}

This example describes how gene expression in Actinomycetes can be modulated. The actIORF1 gene was selected for these experiments.

The codon-optimised Cas9 (SEQ ID NO: 1) was mutated to a catalytically dead version, which was done by point mutation of D10A and H840A. This version of Cas 9 was called dCas 9 and is lacking endonuclease activity (Figure 11).

Three sgRNAs targeting the non-template strand DNA and three sgRNAs targeting the template strand DNA of the coding region of actIORF1 gene were selected. Another set of three sgRNAs targeting the template / non-template strand of the promoter region of actIORF1 gene (total 12) were chosen (Table 3 ). In this example, a catalytically dead Cas9 (dCas9) having both mutations D10A and H840A was used.

The cloning strategy for sgRNA was the same as for the CRISPR-Cas9 system for deletion described above. The conjugates were streaked on the ISP2 agar containing 1 
$\mu \mathrm{g} / \mathrm{ml}$ thiostrepton (the inducer for dCas9), $50 \mu \mathrm{g} / \mathrm{ml}$ apramycin, and $50 \mu \mathrm{g} / \mathrm{ml}$ nalidixic acid and incubated for 7 days at $30^{\circ} \mathrm{C}$.

Actinorhodin production was abolished or dramatically reduced (Figure 12) in clones encoding sgRNAs targeted on the promoter region of actlORF1 gene, independently of which of the template strand DNA or non-template strand DNA was targeted. In contrast, loss or decrease of actinorhodin production in clones carrying sgRNAs that target the coding region, was only observed in the clones with sgRNAs directed to the nontemplate strand (Figure 12).

10

To provoke the loss of the pCRISPR-Cas9 plasmid, the temperature of the incubaton was raised to $37^{\circ} \mathrm{C}$ for $24 \mathrm{~h}$, before transferring the cultures to fresh ISP2 plates without antibiotics and incubating for another 5 days at $37^{\circ} \mathrm{C}$. The previously red clones began to turn blue (Figure 12), indicating that the repression of actinorhodin biosynthesis by the CRISPR-dCas9 system was abrogated and the related gene started to express.

This example shows that gene expression can be modulated in actinomycetes by using the present system.

\section{Sequences}

\section{SEQ ID NO}

1

2

3

4

5

6

7

8

9

10
Name
Codon-optimised Cas9

Cas9 protein

cas 9

Actlorf1-1 NT

Actlorf1-2 T

Actlorf1-3 T

Actlorf1-4 T

Actlorf1-5 T

Actlorf1-6 T

Actvb-1 NT
Description

DNA sequence, codon-

optimised for Streptomy-

ces coelicolor

Translation of SEQ ID

NO: 1

DNA from S. pyogenes

Table 3

Table 3

Table 3

Table 3

Table 3

Table 3

Table 3 
Actvb-2 NT

Actvb-3 T

Actvb-4 T

Actvb-5 NT

Actvb-6 NT

orf1p-S1 T

orf1p-S3 T

orf1p-S5 T

orf1p-A1 NT

orf1p-A4 NT

orf1p-A5 NT

Actlorf1-7 NT

Actlorf1-8 NT

Actlorf1-F1

Actlorf1-F2

Actlorf1-F3

Actlorf1-F4

Actlorf1-F5

Actlorf1-F6

Actlorf1-F7

Actlorf1-F8

ActVB-F1

ActVB-F2

ActVB-F3

ActVB-F4

ActVB-F5

ActVB-F6

orf1p-S1 T-F

orf1p-S3 T-F

orf1p-S5 T-F

orf1p-A1 NT-F

orf1p-A4 NT-F

orf1p-A5 NT-F

sgRNA-R

gRNA check-F
Table 3

Table 3

Table 3

Table 3

Table 3

Table 3

Table 3

Table 3

Table 3

Table 3

Table 3

Table 3

Table 3

Table 4

Table 4

Table 4

Table 4

Table 4

Table 4

Table 4

Table 4

Table 4

Table 4

Table 4

Table 4

Table 4

Table 4

Table 4

Table 4

Table 4

Table 4

Table 4

Table 4

Table 4

Table 4 


\begin{tabular}{|c|c|c|}
\hline 46 & gRNA check-R & Table 4 \\
\hline 47 & orf1-5F & Table 4 \\
\hline 48 & orf1-5'R & Table 4 \\
\hline 49 & orf1-3‘F & Table 4 \\
\hline 50 & orf1-3'R & Table 4 \\
\hline 51 & VB-5'F & Table 4 \\
\hline 52 & VB-5'R & Table 4 \\
\hline 53 & VB-3‘F & Table 4 \\
\hline 54 & VB-3‘R & Table 4 \\
\hline 55 & VB-check-F & Table 4 \\
\hline 56 & VB-check-R & Table 4 \\
\hline 57 & ORF1-check-F & Table 4 \\
\hline 58 & ORF1-check-R & Table 4 \\
\hline 59 & CAS9-check-F & Table 4 \\
\hline 60 & CAS9-check-R & Table 4 \\
\hline 61 & ScaligD-F & Table 4 \\
\hline 62 & ScaligD-R & Table 4 \\
\hline 63 & orf1-6 ligD test-F & Table 4 \\
\hline 64 & orf1-6 ligD test-R & Table 4 \\
\hline 65 & vb2 ligD test-F & Table 4 \\
\hline 66 & vb2 ligD test-R & Table 4 \\
\hline 67 & core guide RNA & Example 1 \\
\hline 68 & sgRNA scaffold & Example 1 \\
\hline 69 & Target-specific Fw primer & Table 3 \\
\hline 70 & Translation of SEQ ID NO: 3 & \\
\hline 71 & S. carneus ligD DNA & \\
\hline 72 & Translation of SEQ ID NO: & \\
\hline & 71 & \\
\hline
\end{tabular}

SEQ ID NO: 1 Codon-optimised Cas 9 ATGGACAAGAAGTACTCCATCGGCCTCGACATCGGCACCAACTCCGTGGGCTGG 5 GCGGTCATCACCGACGAGTACAAGGTCCCCTCCAAGAAGTTCAAGGTCCTGGGC AACACCGACCGGCACTCGATCAAGAAGAACCTGATCGGCGCCCTGCTCTTCGAC 
AGCGGCGAGACCGCCGAGGCGACCCGCCTGAAGCGGACCGCGCGTCGCCGCTA CACCCGGCGCAAGAACCGCATCTGCTACCTGCAGGAAATCTTCTCCAACGAGATG GCCAAGGTGGACGACTCGTTCTTCCACCGCCTGGAGGAGAGCTTCCTGGTGGAG GAGGACAAGAAGCACGAGCGCCACCCGATCTTCGGCAACATCGTGGACGAGGTG GCCTACCACGAGAAGTACCCCACCATCTACCACCTCCGCAAGAAGCTGGTGGACT CGACCGACAAGGCGGACCTGCGGCTCATCTACCTGGCCCTCGCGCACATGATCA AGTTCCGCGGCCACTTCCTCATCGAGGGCGACCTGAACCCGGACAACTCCGACG TGGACAAGCTCTTCATCCAGCTGGTGCAGACCTACAACCAGCTGTTCGAGGAGAA CCCCATCAACGCCAGCGGCGTGGACGCCAAGGCGATCCTCTCCGCGCGCCTGA

10 GCAAGTCCCGGCGCCTGGAGAACCTCATCGCCCAGCTGCCGGGCGAGAAGAAG AACGGCCTCTTCGGCAACCTGATCGCGCTGTCGCTCGGCCTGACCCCCAACTTC AAGAGCAACTTCGACCTGGCCGAGGACGCGAAGCTCCAGCTGTCCAAGGACACC TACGACGACGACCTGGACAACCTGCTCGCCCAGATCGGCGACCAGTACGCGGAC CTCTTCCTGGCCGCGAAGAACCTCTCGGACGCCATCCTGCTCAGCGACATCCTG

15 CGGGTCAACACCGAGATCACCAAGGCCCCGCTGTCGGCGAGCATGATCAAGCGG TACGACGAGCACCACCAGGACCTGACCCTGCTCAAGGCCCTCGTGCGCCAGCAG CTGCCCGAGAAGTACAAGGAAATCTTCTTCGACCAGTCCAAGAACGGCTACGCCG GCTACATCGACGGCGGCGCGTCGCAGGAGGAGTTCTACAAGTTCATCAAGCCGA TCCTGGAGAAGATGGACGGCACCGAGGAGCTGCTCGTCAAGCTGAACCGCGAGG 20 ACCTGCTCCGCAAGCAGCGGACCTTCGACAACGGCTCCATCCCGCACCAGATCC ACCTGGGCGAGCTCCACGCCATCCTCCGGCGCCAGGAGGACTTCTACCCCTTCC TGAAGGACAACCGCGAGAAGATCGAGAAGATCCTGACCTTCCGCATCCCGTACTA CGTCGGCCCCCTGGCCCGCGGCAACTCCCGGTTCGCGTGGATGACCCGGAAGT CGGAGGAGACCATCACCCCGTGGAACTTCGAGGAGGTCGTGGACAAGGGCGCG

25 TCCGCGCAGTCGTTCATCGAGCGCATGACCAACTTCGACAAGAACCTCCCGAACG AGAAGGTCCTGCCCAAGCACTCCCTGCTCTACGAGTACTTCACCGTGTACAACGA GCTGACCAAGGTCAAGTACGTGACCGAGGGCATGCGGAAGCCGGCCTTCCTGTC GGGCGAGCAGAAGAAGGCGATCGTGGACCTGCTCTTCAAGACCAACCGCAAGGT CACCGTGAAGCAGCTGAAGGAGGACTACTTCAAGAAGATCGAGTGCTTCGACTCC

30 GTCGAGATCAGCGGCGTGGAGGACCGCTTCAACGCCTCCCTGGGCACCTACCAC GACCTGCTCAAGATCATCAAGGACAAGGACTTCCTCGACAACGAGGAGAACGAG GACATCCTGGAGGACATCGTCCTCACCCTGACCCTCTTCGAGGACCGCGAGATG ATCGAGGAGCGGCTCAAGACCTACGCCCACCTGTTCGACGACAAGGTGATGAAG CAGCTGAAGCGTCGCCGCTACACCGGCTGGGGCCGCCTCTCCCGGAAGCTGATC AACGGCATCCGGGACAAGCAGAGCGGCAAGACCATCCTGGACTTCCTCAAGTCC 
GACGGCTTCGCCAACCGCAACTTCATGCAGCTCATCCACGACGACAGCCTGACCT TCAAGGAGGACATCCAGAAGGCCCAGGTCTCGGGCCAGGGCGACAGCCTCCAC GAGCACATCGCCAACCTGGCGGGCTCCCCGGCGATCAAGAAGGGCATCCTCCAG ACCGTCAAGGTCGTGGACGAGCTGGTCAAGGTGATGGGCCGCCACAAGCCCGA GAACATCGTGATCGAGATGGCCCGGGAGAACCAGACCACCCAGAAGGGCCAGAA GAACTCGCGCGAGCGGATGAAGCGGATCGAGGAGGGCATCAAGGAGCTCGGCA GCCAGATCCTGAAGGAGCACCCGGTCGAGAACACCCAGCTGCAGAACGAGAAGC TGTACCTCTACTACCTGCAGAACGGCCGCGACATGTACGTGGACCAGGAGCTCG ACATCAACCGGCTGTCCGACTACGACGTGGACCACATCGTGCCGCAGTCCTTCCT

10 GAAGGACGACTCGATCGACAACAAGGTCCTGACCCGCTCGGACAAGAACCGGGG CAAGTCCGACAACGTGCCCTCGGAGGAGGTCGTGAAGAAGATGAAGAACTACTG GCGCCAGCTGCTCAACGCCAAGCTCATCACCCAGCGCAAGTTCGACAACCTGAC CAAGGCCGAGCGGGGCGGCCTGAGCGAGCTCGACAAGGCGGGCTTCATCAAGC GCCAGCTGGTCGAGACCCGGCAGATCACCAAGCACGTGGCCCAGATCCTGGACT

15 CCCGGATGAACACCAAGTACGACGAGAACGACAAGCTGATCCGCGAGGTCAAGG TGATCACCCTCAAGAGCAAGCTGGTCTCCGACTTCCGCAAGGACTTCCAGTTCTA CAAGGTCCGGGAGATCAACAACTACCACCACGCCCACGACGCGTACCTGAACGC CGTCGTGGGCACCGCGCTGATCAAGAAGTACCCGAAGCTGGAGTCCGAGTTCGT CTACGGCGACTACAAGGTCTACGACGTGCGCAAGATGATCGCCAAGAGCGAGCA 20 GGAGATCGGCAAGGCCACCGCGAAGTACTTCTTCTACTCCAACATCATGAACTTC TTCAAGACCGAGATCACCCTGGCCAACGGCGAGATCCGCAAGCGGCCCCTGATC GAGACCAACGGCGAGACCGGCGAGATCGTCTGGGACAAGGGCCGCGACTTCGC CACCGTCCGGAAGGTGCTGTCGATGCCGCAGGTCAACATCGTGAAGAAGACCGA GGTGCAGACCGGCGGCTTCAGCAAGGAGTCCATCCTCCCCAAGCGCAACAGCGA

25 CAAGCTGATCGCCCGGAAGAAGGACTGGGACCCGAAGAAGTACGGCGGCTTCGA CAGCCCCACCGTCGCCTACTCCGTGCTGGTCGTGGCGAAGGTCGAGAAGGGCAA GAGCAAGAAGCTGAAGTCCGTGAAGGAGCTGCTCGGCATCACCATCATGGAGCG CTCCTCGTTCGAGAAGAACCCGATCGACTTCCTGGAGGCCAAGGGCTACAAGGA GGTCAAGAAGGACCTCATCATCAAGCTGCCCAAGTACAGCCTGTTCGAGCTGGAG

30 AACGGCCGCAAGCGGATGCTCGCCTCCGCGGGCGAGCTGCAGAAGGGCAACGA GCTGGCCCTCCCGTCGAAGTACGTCAACTTCCTGTACCTCGCGTCCCACTACGAG AAGCTGAAGGGCTCGCCCGAGGACAACGAGCAGAAGCAGCTCTTCGTGGAGCAG CACAAGCACTACCTGGACGAGATCATCGAGCAGATCAGCGAGTTCAGCAAGCGC GTCATCCTGGCCGACGCGAACCTCGACAAGGTGCTGTCCGCCTACAACAAGCAC 35 CGCGACAAGCCGATCCGGGAGCAGGCGGAGAACATCATCCACCTGTTCACCCTC 
ACCAACCTGGGCGCCCCCGCCGCGTTCAAGTACTTCGACACCACCATCGACCGC AAGCGGTACACCTCCACCAAGGAGGTCCTCGACGCGACCCTGATCCACCAGAGC ATCACCGGCCTGTACGAGACCCGCATCGACCTGTCCCAGCTCGGCGGCGACTGA

SEQ ID NO: 2 - Protein sequence for codon-optimised Cas9:

MDKKYSIGLDIGTNSVGWAVITDEYKVPSKKFKVLGNTDRHSIKKNLIGALLFDSGETA EATRLKRTARRRYTRRKNRICYLQEIFSNEMAKVDDSFFHRLEESFLVEEDKKHERHP IFGNIVDEVAYHEKYPTIYHLRKKLVDSTDKADLRLIYLALAHMIKFRGHFLIEGDLNPDN SDVDKLFIQLVQTYNQLFEENPINASGVDAKAILSARLSKSRRLENLIAQLPGEKKNGLF GNLIALSLGLTPNFKSNFDLAEDAKLQLSKDTYDDDLDNLLAQIGDQYADLFLAAKNLS DAILLSDILRVNTEITKAPLSASMIKRYDEHHQDLTLLKALVRQQLPEKYKEIFFDQSKN GYAGYIDGGASQEEFYKFIKPILEKMDGTEELLVKLNREDLLRKQRTFDNGSIPHQIHL GELHAILRRQEDFYPFLKDNREKIEKILTFRIPYYVGPLARGNSRFAWMTRKSEETITP WNFEEVVDKGASAQSFIERMTNFDKNLPNEKVLPKHSLLYEYFTVYNELTKVKYVTEG MRKPAFLSGEQKKAIVDLLFKTNRKVTVKQLKEDYFKKIECFDSVEISGVEDRFNASLG TYHDLLKIIKDKDFLDNEENEDILEDIVLTLTLFEDREMIEERLKTYAHLFDDKVMKQLKR RRYTGWGRLSRKLINGIRDKQSGKTILDFLKSDGFANRNFMQLIHDDSLTFKEDIQKA QVSGQGDSLHEHIANLAGSPAIKKGILQTVKVVDELVKVMGRHKPENIVIEMARENQT TQKGQKNSRERMKRIEEGIKELGSQILKEHPVENTQLQNEKLYLYYLQNGRDMYVDQ

20 ELDINRLSDYDVDHIVPQSFLKDDSIDNKVLTRSDKNRGKSDNVPSEEVVKKMKNYW RQLLNAKLITQRKFDNLTKAERGGLSELDKAGFIKRQLVETRQITKHVAQILDSRMNTK YDENDKLIREVKVITLKSKLVSDFRKDFQFYKVREINNYHHAHDAYLNAVVGTALIKKY PKLESEFVYGDYKVYDVRKMIAKSEQEIGKATAKYFFYSNIMNFFKTEITLANGEIRKRP LIETNGETGEIVWDKGRDFATVRKVLSMPQVNIVKKTEVQTGGFSKESILPKRNSDKLI 25 ARKKDWDPKKYGGFDSPTVAYSVLVVAKVEKGKSKKLKSVKELLGITIMERSSFEKNP IDFLEAKGYKEVKKDLIIKLPKYSLFELENGRKRMLASAGELQKGNELALPSKYVNFLYL ASHYEKLKGSPEDNEQKQLFVEQHKHYLDEIIEQISEFSKRVILADANLDKVLSAYNKH RDKPIREQAENIIHLFTLTNLGAPAAFKYFDTTIDRKRYTSTKEVLDATLIHQSITGLYET RIDLSQLGGD.

SEQ ID NO: 3 S. pyogenes cas9

ATGGATAAGAAATACTCAATAGGCTTAGATATCGGCACAAATAGCGTCGGATGGG CGGTGATCACTGATGAATATAAGGTTCCGTCTAAAAAGTTCAAGGTTCTGGGAAAT ACAGACCGCCACAGTATCAAAAAAATCTTATAGGGGCTCTTTTATTTGACAGTGG AGAGACAGCGGAAGCGACTCGTCTCAAACGGACAGCTCGTAGAAGGTATACACG 
TCGGAAGAATCGTATTTGTTATCTACAGGAGATTTTTTCAAATGAGATGGCGAAAG TAGATGATAGTTTCTTTCATCGACTTGAAGAGTCTTTTTTGGTGGAAGAAGACAAG AAGCATGAACGTCATCCTATTTTTGGAAATATAGTAGATGAAGTTGCTTATCATGA GAAATATCCAACTATCTATCATCTGCGAAAAAAATTGGTAGATTCTACTGATAAAGC GGATTTGCGCTTAATCTATTTGGCCTTAGCGCATATGATTAAGTTTCGTGGTCATTT TTTGATTGAGGGAGATTTAAATCCTGATAATAGTGATGTGGACAAACTATTTATCCA GTTGGTACAAACCTACAATCAATTATTTGAAGAAAACCCTATTAACGCAAGTGGAG TAGATGCTAAAGCGATTCTTTCTGCACGATTGAGTAAATCAAGACGATTAGAAAAT CTCATTGCTCAGCTCCCCGGTGAGAAGAAAAATGGCTTATTTGGGAATCTCATTGC

10 TTTGTCATTGGGTTTGACCCCTAATTTTAAATCAAATTTTGATTTGGCAGAAGATGC TAAATTACAGCTTTCAAAAGATACTTACGATGATGATTTAGATAATTTATTGGCGCA AATTGGAGATCAATATGCTGATTTGTTTTTGGCAGCTAAGAATTTATCAGATGCTAT TTTACTTTCAGATATCCTAAGAGTAAATACTGAAATAACTAAGGCTCCCCTATCAGC TTCAATGATTAAACGCTACGATGAACATCATCAAGACTTGACTCTTTTAAAAGCTTT

15 AGTTCGACAACAACTTCCAGAAAAGTATAAAGAAATCTTTTTTGATCAATCAAAAAA CGGATATGCAGGTTATATTGATGGGGGAGCTAGCCAAGAAGAATTTTATAAATTTA TCAAACCAATTTTAGAAAAAATGGATGGTACTGAGGAATTATTGGTGAAACTAAAT CGTGAAGATTTGCTGCGCAAGCAACGGACCTTTGACAACGGCTCTATTCCCCATC AAATTCACTTGGGTGAGCTGCATGCTATTTTGAGAAGACAAGAAGACTTTTATCCA 20 TTTTTAAAAGACAATCGTGAGAAGATTGAAAAAATCTTGACTTTTCGAATTCCTTAT TATGTTGGTCCATTGGCGCGTGGCAATAGTCGTTTTGCATGGATGACTCGGAAGT CTGAAGAAACAATTACCCCATGGAATTTTGAAGAAGTTGTCGATAAAGGTGCTTCA GCTCAATCATTTATTGAACGCATGACAAACTTTGATAAAAATCTTCCAAATGAAAAA GTACTACCAAAACATAGTTTGCTTTATGAGTATTTTACGGTTTATAACGAATTGACA 25 AAGGTCAAATATGTTACTGAAGGAATGCGAAAACCAGCATTTCTTTCAGGTGAACA GAAGAAAGCCATTGTTGATTTACTCTTCAAAACAAATCGAAAAGTAACCGTTAAGC AATTAAAAGAAGATTATTTCAAAAAAATAGAATGTTTTGATAGTGTTGAAATTTCAG GAGTTGAAGATAGATTTAATGCTTCATTAGGTACCTACCATGATTTGCTAAAAATTA TTAAAGATAAAGATTTTTTGGATAATGAAGAAAATGAAGATATCTTAGAGGATATTG 30 TTTTAACATTGACCTTATTTGAAGATAGGGAGATGATTGAGGAAAGACTTAAAACAT ATGCTCACCTCTTTGATGATAAGGTGATGAAACAGCTTAAACGTCGCCGTTATACT GGTTGGGGACGTTTGTCTCGAAAATTGATTAATGGTATTAGGGATAAGCAATCTGG CAAAACAATATTAGATTTTTTGAAATCAGATGGTTTTGCCAATCGCAATTTTATGCA GCTGATCCATGATGATAGTTTGACATTTAAAGAAGACATTCAAAAAGCACAAGTGT 35 CTGGACAAGGCGATAGTTTACATGAACATATTGCAAATTTAGCTGGTAGCCCTGCT 
ATTAAAAAAGGTATTTTACAGACTGTAAAAGTTGTTGATGAATTGGTCAAAGTAATG GGGCGGCATAAGCCAGAAAATATCGTTATTGAAATGGCACGTGAAAATCAGACAA CTCAAAAGGGCCAGAAAAATTCGCGAGAGCGTATGAAACGAATCGAAGAAGGTAT CAAAGAATTAGGAAGTCAGATTCTTAAAGAGCATCCTGTTGAAAATACTCAATTGC AAAATGAAAAGCTCTATCTCTATTATCTCCAAAATGGAAGAGACATGTATGTGGAC CAAGAATTAGATATTAATCGTTTAAGTGATTATGATGTCGATCACATTGTTCCACAA AGTTTCCTTAAAGACGATTCAATAGACAATAAGGTCTTAACGCGTTCTGATAAAAAT CGTGGTAAATCGGATAACGTTCCAAGTGAAGAAGTAGTCAAAAAGATGAAAAACTA TTGGAGACAACTTCTAAACGCCAAGTTAATCACTCAACGTAAGTTTGATAATTTAAC GAAAGCTGAACGTGGAGGTTTGAGTGAACTTGATAAAGCTGGTTTTATCAAACGC CAATTGGTTGAAACTCGCCAAATCACTAAGCATGTGGCACAAATTTTGGATAGTCG CATGAATACTAAATACGATGAAAATGATAAACTTATTCGAGAGGTTAAAGTGATTAC CTTAAAATCTAAATTAGTTTCTGACTTCCGAAAAGATTTCCAATTCTATAAAGTACG TGAGATTAACAATTACCATCATGCCCATGATGCGTATCTAAATGCCGTCGTTGGAA CTGCTTTGATTAAGAAATATCCAAAACTTGAATCGGAGTTTGTCTATGGTGATTATA AAGTTTATGATGTTCGTAAAATGATTGCTAAGTCTGAGCAAGAAATAGGCAAAGCA ACCGCAAAATATTTCTTTTACTCTAATATCATGAACTTCTTCAAAACAGAAATTACA CTTGCAAATGGAGAGATTCGCAAACGCCCTCTAATCGAAACTAATGGGGAAACTG GAGAAATTGTCTGGGATAAAGGGCGAGATTTTGCCACAGTGCGCAAAGTATTGTC CATGCCCCAAGTCAATATTGTCAAGAAAACAGAAGTACAGACAGGCGGATTCTCC AAGGAGTCAATTTTACCAAAAAGAAATTCGGACAAGCTTATTGCTCGTAAAAAAGA CTGGGATCCAAAAAAATATGGTGGTTTTGATAGTCCAACGGTAGCTTATTCAGTCC TAGTGGTTGCTAAGGTGGAAAAAGGGAAATCGAAGAAGTTAAAATCCGTTAAAGA GTTACTAGGGATCACAATTATGGAAAGAAGTTCCTTTGAAAAAAATCCGATTGACT

25 TTTTAGAAGCTAAAGGATATAAGGAAGTTAAAAAAGACTTAATCATTAAACTACCTA AATATAGTCTTTTTGAGTTAGAAAACGGTCGTAAACGGATGCTGGCTAGTGCCGG AGAATTACAAAAAGGAAATGAGCTGGCTCTGCCAAGCAAATATGTGAATTTTTTAT ATTTAGCTAGTCATTATGAAAAGTTGAAGGGTAGTCCAGAAGATAACGAACAAAAA CAATTGTTTGTGGAGCAGCATAAGCATTATTTAGATGAGATTATTGAGCAAATCAG 30 TGAATTTTCTAAGCGTGTTATTTTAGCAGATGCCAATTTAGATAAAGTTCTTAGTGC ATATAACAAACATAGAGACAAACCAATACGTGAACAAGCAGAAAATATTATTCATTT ATTTACGTTGACGAATCTTGGAGCTCCCGCTGCTTTTAAATATTTTGATACAACAAT TGATCGTAAACGATATACGTCTACAAAAGAAGTTTTAGATGCCACTCTTATCCATCA ATCCATCACTGGTCTTTATGAAACACGCATTGATTTGAGTCAGCTAGGAGGTGACT $\mathrm{GA}$ 
SEQ ID NO: 71. S. carneus ligD

ATCGAGGTCCGGCTGAGCAACCTGGACAAGGTGCTCTATCCGGCGACCGGCACC ACCAAGGGCGAGGTCATCGAGTACTACGCCGAAATCGCCCCGGCGATGCTGCCG CATATCGCGGGCCGGCCGATCACCCGGAAACGGTGGCCGAACGGTGTCGCCGA ATCGTCGTTCTTCGAGAAGAACCTCGGCGCGGGTACACCGTCGTGGCTACCGCG CCGTGCCCAGGAACATTCCGACCGCACCGCGCACTATCCGGTGATCTCGTCGCA GGCCGGCCTGGTCTGGCTGGGTCAGCAGGCCGCCCTGGAGATCCACGTACCGC AATGGCGCTTCGACGGCGATGCGCGCGGACCCGCGACGCGGCTGGTGTTCGAT

10 CTCGATCCCGGCCCCGGCGCGGGACTGCCCGAATGCGCGCGGGTGGCGCTCGG GGTGCGGGATATGGTCGCCGAAATCGGGATGCGCGCGTTCCCGCTGACCAGCG GTAGCAAAGGTATCCACCTGTACGTCCCGCTGGACCGGGTGCTGAGCCCCGGCG GGGCGTCCACGGTGGCCAAACAGGTCGCCGCGAATCTGGAGAAACTCCTTCCCG ACCTGGTCACCGCCACCATCGCGAAGAGTGTGCGGGCCGGGAAGGTGTTCCTGG ACTGGAGTCAGAACAACCCGTCCAAGACGACCATCGCACCGTATTCGCTGCGCG GCCGCGAGCAGCCGAACGTCGCCGCACCACGCCACTGGGCGGAGCTCGAGGAC GCCCGTGAACTGCGGCAGCTGCGGTTCGACGAAGTTCTGGAGCGTTATCGGTCC GAGGGTGATCTGCTGGCCGGCCTGGATACACCCCTGAACGACGCGTTGACGAAA TACCGATCGATGCGTGACCCGGCGCGTACACCGGAGCCGGTACCGCCGCATTCG CCCCGGCCCGGCCCCGGTGACCGCTATGTCGTCCACGAACACCACGCCCGGCG GTTGCACTGGGATGTGCGGTTGGAACGCGACGGGGTGCTGGTGTCGTGGGCGG TGCCCAAGGGGCCGCCGGAAAGCACCCGGCAGAATCGGCTCGCCGTGCACACC GAGGACCACCCGCTGGAATACCTGGACTTCCACGGCACGATCCCGGCCGGCGA GTACGGGGCAGGGGAGCTGTCGGTCTGGGATACCGGCACCTACCGCGCCGAGA AATGGCGCGACGACGAGGTGATCGTGGTTTTCCGGGGCGAGCGGCTCAACGGC CGGTACGCCATGATCCGGACCGAGGGCGATCAATGGCTGATGCATCTCATGAAG GACCAGCCCGCGACCGGGGAACTGCCGCGTGGACTCACCCCCATGCTGGCCAC CAGTGGCGAAGTGGCCGGGCTGCCGGACTCGGAGTGGGCGTTCGAACGTAAAT GGGACGGATACCGGCTGCTCGTCGAAATCGATGCCGGCGAAATGCGGCTGCGCA

30 GCCGGGCCGGTAACGACGTCACCGCGCGCTATCCCCAGTTGTCGGTGCTGGCC GAGGAGCTGGCCGACCATCAGGTGATACTCGACGGTGAGCTCATCGTCCGCGGC CCCGACGGCGCGGTGAATATCGCGCTGTTGAAGGCGAATCCGCGGCGCGCCGA ATTCCTGGCGTTCGATCTGCTGTTCCTCGACGGCACTTCACTGCTGCGCAAACGC TACCGCGATCGGCGGCACGTGCTCGAAGCGCTGGCCGCGACCACCACCGAACT

35 CCGGGTGCCACCGCGCTATGAGGGCGACGGCACCGAGGCCCTGCACCGCAGCG 
AAGAAGATGGCGCCGAGGGCGTGATCGCCAAACGGCTGGATTCGGTGTATCTGC CCGGGACCCGCGGGCATTCGTGGGTGAAGCACCGGAACTGGCGTACCCAGGAG GTGGTGATCGGGGGTATGCGGCGCAGTAAGGCGCGACCGTTCGCCTCGTTGCTG GTCGGGATACCGGCCGAGGACGGCCTGGTGTATGCGGGCCGGGTCGGGACCGG GTTCGACGAAGCGGGGATGACCGAACTCGCGGCCCGGCTGCGCCGGTCGGAAC GTAAGACGCCGCCGTTCACCAACGAGATGTCGGCCGATGAACTCCGGGACGCGA TCTGGGTGACACCGAAGATCAAAGGCACTGTTCGCTACATGGATTGGACCGACG GCGGACGCTTCTGGCATCCTGCCTGGCTCGGCGAGGTGTGA

\section{References}

Bentley SD, Chater KF, Cerdeno-Tarraga AM, Challis GL, Thomson NR, James KD, Harris DE, Quail MA, Kieser H, Harper D, Bateman A, Brown S, Chandra G, Chen CW,

15 Collins M, Cronin A, Fraser A, Goble A, Hidalgo J, Hornsby T, Howarth S, Huang CH, Kieser T, Larke L, Murphy L, Oliver K, O'Neil S, Rabbinowitsch E, Rajandream MA, Rutherford K, Rutter S, Seeger K, Saunders D, Sharp S, Squares R, Squares S, Taylor K, Warren T, Wietzorrek A, Woodward J, Barrell BG, Parkhill J, Hopwood DA. 2002. Complete genome sequence of the model actinomycete Streptomyces coelicolor A3(2). $20 \quad$ Nature 417:141-147.

Cobb RE, Wang Y, Zhao H. 2014. High-Efficiency Multiplex Genome Editing of Streptomyces Species Using an Engineered CRISPR/Cas System. ACS synthetic biology.

Bikard, D., Euler, C. W., Jiang, W. Y., Nussenzweig, P. M., Goldberg, G. W., Duportet, X., Fischetti, V. A., and Marraffini, L. A. 2014. Exploiting CRISPR-Cas nucleases to produce sequence-specific antimicrobials. Nat.Biotechnol. 32:1146-1150. using efficiently delivered RNA-guided nucleases. Nat. Biotechnol. 32:1141-1145.

Gomaa, A. A., Klumpe, H. E., Luo, M. L., Selle, K., Barrangou, R., and Beisel, C. L. 2014. Programmable removal of bacterial strains by use of genome targeting CRISPRCas systems. Mbio 5, e00928-13. DOI:10.1128/mBio.00928-13. 
Hilker R, Stadermann KB, Doppmeier D, Kalinowski J, Stoye J, Straube J, Winnebald J, Goesmann A. 2014. ReadXplorer--visualization and analysis of mapped sequences. Bioinformatics 30:2247-2254.

$5 \quad$ Huang $\mathrm{H}$, Zheng G, Jiang $\mathrm{W}$, Hu H, Lu Y. 2015. One-step high-efficiency CRISPR/Cas9-mediated genome editing in Streptomyces. Acta Biochim Biophys Sin (Shanghai).

Li H, Durbin R. 2009. Fast and accurate short read alignment with Burrows-Wheeler 10 transform. Bioinformatics 25:1754-1760.

MacNeil DJ, Occi JL, Gewain KM, MacNeil T, Gibbons PH, Ruby CL, Danis SJ. 1992. Complex organization of the Streptomyces avermitilis genes encoding the avermectin polyketide synthase. Gene 115:119-125.

Muth G, Nussbaumer B, Wohlleben W, Puhler A. 1989. A Vector System with Temperature-Sensitive Replication for Gene Disruption and Mutational Cloning in Streptomycetes. Molecular \& General Genetics 219:341-348.

20 Qi, L. S., Larson, M. H., Gilbert, L. A., Doudna, J. A., Weissman, J. S., Arkin, A. P., and Lim, W. A. 2013. Repurposing CRISPR as an RNA-guided platform for sequencespecific control of gene expression. Cell 152:1173-1183.

Rutherford K, Parkhill J, Crook J, Horsnell T, Rice P, Rajandream MA, Barrell B. 2000. Artemis: sequence visualization and annotation. Bioinformatics 16:944-945.

\section{Items}

1. A method for generating at least one deletion around at least one target nucleic acid sequence comprised within a host cell having a non-homologous end-joining (NHEJ) pathway which is at least partly deficient, said method comprising the step of inducing a CRISPR-Cas 9 system in a host cell, wherein said CRISPR-Cas9 system is able to generate at least one break in said at least one target nucleic acid sequence and wherein the 
CRISPR-Cas9 system comprises a Cas9 nuclease and at least one guiding means,

thereby generating at least one deletion around said at least one target nucleic acid sequence, wherein said at least one deletion is a deletion of at least $1 \mathrm{bp}$.

2. The method of item 1, further comprising the step of determining the size of the deletion.

3. The method of any one of the preceding items, wherein said at least one deletion is one deletion.

4. The method of any one of the preceding items, wherein said at least one target nucleic acid sequence is one target nucleic acid sequence.

5. The method of any one of the preceding items, wherein the guiding means comprises at least one sgRNA and/or at least one crRNA/tracrRNA set.

6. The method of any one of the preceding items, wherein the host cell is an archae, a prokaryotic cell or a eukaryotic cell.

7. The method of any one of the preceding items, wherein the NHEJ pathway of said host cell comprises at least one of four activities defined as:

- a DNA-binding activity,

- a primase activity,

- a ligase activity.

- a polymerase activity.

8. The method of item 7 , wherein at least one is two or three.

9. The method of any one of items 7 or 8 , wherein said host cell is naturally lacking at least one said four activities or wherein at least one of said four activities has been inactivated. 
10. The method of any one of the preceding items, wherein the host cell is selected from the group consisting of actinobacteria.

11. The method of any one of the preceding items, wherein the host cell is selected from the group consisting of Actinomycetales, such as Streptomyces sp., Amycolatopsis sp. or Saccharopolyspora sp.

12. The method of any one of the preceding items, wherein the host cell is selected from the group consisting of Streptomyces coelicolor, Streptomyces avermitilis, Streptomyces aureofaciens, Streptomyces griseus, Streptomyces parvulus, Streptomyces albus, Streptomyces vinaceus, Streptomyces acrimycinis, Streptomyces calvuligerus, Streptomyces lividans, Streptomyces limosus, Streptomyces rubiqinosis, Streptomyces azureus, Streptomyces glaucenscens, Streptomyces rimosus, Streptomyces violaceoruber, Streptomyces kanamyceticus, Amycolatopsis orientalis, Amycolatopsis mediterranei and Saccharopolyspora erythraea.

13. The method of any one of the preceding items, wherein the at least one target nucleic acid sequence is comprised within a secondary metabolite biosynthetic gene.

14. The method of any one of the preceding items, wherein the at least one target nucleic acid sequence is comprised within a gene cluster such as a secondary metabolite gene cluster.

15. The method of any one of items 13 to 14 , wherein the secondary metabolite is selected from the group consisting of antibiotics, herbicides, anti-cancer agents, immunosuppressants, flavors, parasiticides, enzymes and proteins.

16. The method of any one of items 13 to 15 , wherein the secondary metabolite is an antibiotic selected from the group consisting of apramycin, bacitracin, chloramphenicol cephalosporins, cycloserine, erythromycin, fosfomycin, gentamicin, kanamycin, kirromycin, lassomycin, lincomycin, lysolipin, microbisporicin, neomycin, noviobiocin, nystatin, nitrofurantoin, platensimycin, 
pristinamycins, rifamycin, streptomycin, teicoplanin, tetracycline, tinidazole, ribostamycin, daptomycin, vancomycin, viomycin and virginiamycin.

17. The method of any one of items 13 to 15 , wherein the secondary metabolite is a herbicide selected from the group consisting of bialaphos, resormycin and phosphinothricin.

18. The method of any one of items 13 to 15 , wherein the secondary metabolite is an anti-cancer agent selected from the group consisting of doxorubicin, salinosporamides, aclarubicin, pentostatin, peplomycin, thrazarine and neocarcinostatin.

19. The method of any one of items 13 to 15 , wherein the secondary metabolite is an immunosuppressant selected from the group consisting of rapamycin, FK520, FK506, cyclosporine, ushikulides, pentalenolactone I and hygromy$\operatorname{cin} \mathrm{A}$.

20. The method of any one of items 13 to 15 , wherein the secondary metabolite is a flavor such as geosmin.

20

21. The method of any one of items 13 to 15 , wherein the secondary metabolite is a parasiticide such as an insecticide, an anthelmintic, a larvacide, or an antiprotozoal agent such as spinsad or avermectin.

22. The method of any one of items 1 to 12 , wherein the at least one nucleic acid encodes an enzyme such as a metabolic enzyme selected from the group consisting of an amylase, a protease, a cellulase, a chitinase, a keratinase and a xylanase, a glycosyltransferase, an oxygenase, a hydroxylase, a methyltransferase, a dehydrogenase, a dehydratase.

23. The method of any one of the preceding items, wherein the generation of at least one deletion results in the inactivation of at least one gene.

24. The method of any one of the preceding items, wherein said deletion is a deletion of 1 to $1500000 \mathrm{bp}$, such as 1 to $1200000 \mathrm{bp}$, such as 1 to 
$1000000 \mathrm{bp}$, such as 1 to $500000 \mathrm{bp}$, such as 1 to $400000 \mathrm{bp}$, such as 1 to $300000 \mathrm{bp}$, such as 1 to $200000 \mathrm{bp}$, such as 1 to $100000 \mathrm{bp}$, such as 2 to $75000 \mathrm{bp}$, such as 3 to $50000 \mathrm{bp}$, such as 4 to $40000 \mathrm{bp}$, such as 5 to $30000 \mathrm{bp}$, such as 10 to $20000 \mathrm{bp}$, such as 25 to $10000 \mathrm{bp}$, such as 50 to $9000 \mathrm{bp}$, such as 75 to $8000 \mathrm{bp}$, such as 100 to $7000 \mathrm{bp}$, such as 150 to $6000 \mathrm{bp}$, such as 200 to $5000 \mathrm{bp}$, such as 250 to $4000 \mathrm{bp}$, such as 300 to $3000 \mathrm{bp}$, such as 400 to $2000 \mathrm{bp}$, such as 500 to $1000 \mathrm{bp}$, such as 600 to $900 \mathrm{bp}$, such as 700 to $800 \mathrm{bp}$.

25. The method of any one of the preceding items, wherein said deletion is a deletion of at least $1 \mathrm{bp}$, such as at least $2 \mathrm{bp}$, such as at least $3 \mathrm{bp}$, such as at least $4 \mathrm{bp}$, such as at least $5 \mathrm{bp}$, such as at least $10 \mathrm{bp}$, such as at least $15 \mathrm{bp}$, such as at least $20 \mathrm{bp}$, such as at least $50 \mathrm{bp}$, such as at least $100 \mathrm{bp}$, such as at least $250 \mathrm{bp}$, such as at least $500 \mathrm{bp}$.

26. The method of any one of the preceding items, wherein said deletion is a deletion of 1 to $100 \mathrm{bp}$, such as 1 to $75 \mathrm{bp}$, such as 1 to $50 \mathrm{bp}$, such as 1 to $40 \mathrm{bp}$, such as 1 to $30 \mathrm{bp}$, such as 1 to $20 \mathrm{bp}$, such as 1 to $10 \mathrm{bp}$, such as 1 to $9 \mathrm{bp}$, such as 1 to $8 \mathrm{bp}$, such as 1 to $7 \mathrm{bp}$, such as 1 to $6 \mathrm{bp}$, such as 1 to $5 \mathrm{bp}$, such as 1 to $4 \mathrm{bp}$, such as 1 to $3 \mathrm{bp}$, such as 1 to $2 \mathrm{bp}$.

27. A method for generating at least one indel around at least one target nucleic acid sequence comprised within a host cell having a non-homologous endjoining (NHEJ) pathway which is at least partly deficient, said method comprising the steps of:

i. restoring the full functionality of the NHEJ pathway in said host cell;

ii. inducing a CRISPR-Cas9 system in said host cell, wherein said CRISPR-Cas9 system is able to generate at least one break in said at least one target nucleic acid sequence and wherein the CRISPRCas 9 system comprises a Cas 9 nuclease and at least one guiding means,

thereby generating at least one indel around said at least one target nucleic acid sequence, wherein said at least one indel is a deletion or insertion of at least1 bp. 
28. The method of item 27 , further comprising the step of determining the size of the indel.

29. The method of any one of items 27 to 28 , wherein said at least one indel is one indel.

30. The method of any one of items 27 to 29 , wherein said at least one target nucleic acid sequence is one target nucleic acid sequence.

10

20

25

15

31. The method of item 30 , wherein the guiding means is a single guide RNA (sgRNA).

32. The method of any one of items 27 to 31 , wherein the host cell is an archaea, a prokaryotic cell or a eukaryotic cell.

33. The method of any one of items 27 to 32 , wherein the NHEJ pathway of said host cell comprises at least one of four activities defined as:

- a DNA-binding activity,

- a primase activity,

- a ligase activity

- a polymerase activity.

34. The method of any one of items 27 to 33 , wherein the NHEJ pathway of said host cell lacks the ligase activity.

35. The method of item 34 , wherein the ligase activity is restored by expression of a functional ligase such as a heterologous ligase.

36. The method of item 35 , wherein the heterologous ligase is derived from an organism selected from the group consisting of: Streptomyces carneus, $M y-$ cobacter tuberculosis, Nocardia spp., Smaragdicoccus niigatensis, Rhodococcus spp., Mycobacterium abscessus, Mycobacterium mageritense and Mycobacterium farcinogenes. 
37. The method of any one of items 27 to 36 , wherein the host cell is selected from the group consisting of actinobacteria.

38. The method of any one of items 27 to 37 , wherein the host cell is selected from the group consisting of Actinomycetales, such as Streptomyces sp., Amycolatopsis sp. or Saccharopolyspora sp..

39. The method of any one of items 27 to 38 , wherein the host cell is selected from the group consisting of Streptomyces coelicolor, Streptomyces avermitilis, Streptomyces aureofaciens, Streptomyces griseus, Streptomyces parvulus, Streptomyces albus, Streptomyces vinaceus, Streptomyces acrimycinis, Streptomyces calvuligerus, Streptomyces lividans, Streptomyces limosus, Streptomyces rubiqinosis, Streptomyces azureus, Streptomyces glaucenscens, Streptomyces rimosus, Streptomyces violaceoruber, Streptomyces kanamyceticus, Amycolatopsis orientalis, Amycolatopsis mediterranei and Saccharopolyspora erythraea.

40. The method of any one of items 27 to 39 , wherein the at least one target nucleic acid sequence is comprised within a secondary metabolite biosynthetic gene.

41. The method of any one of items 27 to 40 , wherein the at least one target nucleic acid sequence is comprised within a gene cluster such as a secondary metabolite gene cluster.

25

42. The method of any one of items 40 to 41 , wherein the secondary metabolite is selected from the group consisting of antibiotics, herbicides, anti-cancer agents, immunosuppressants, flavors, parasiticides, enzymes and proteins.

43. The method of any one of items 40 to 42 , wherein the secondary metabolite is an antibiotic selected from the group consisting of apramycin, bacitracin, chloramphenicol cephalosporins, cycloserine, erythromycin, fosfomycin, gentamicin, kanamycin, kirromycin, lassomycin, lincomycin, lysolipin, microbisporicin, neomycin, noviobiocin, nystatin, nitrofurantoin, platensimycin, 
pristinamycins, rifamycin, streptomycin, teicoplanin, tetracycline, tinidazole, ribostamycin, daptomycin, vancomycin, viomycin, virginiamycin.

44. The method of any one of items 40 to 42 , wherein the secondary metabolite is a herbicide selected from the group consisting of bialaphos, resormycin and phosphinothricin.

45. The method of any one of items 40 to 42 , wherein the secondary metabolite is an anti-cancer agent selected from the group consisting of doxorubicin, salinosporamides, aclarubicin, pentostatin, peplomycin, thrazarine and neocarcinostatin.

46. The method of any one of items 40 to 42 , wherein the secondary metabolite is an immunosuppressant selected from the group consisting of rapamycin, FK520, FK506, cyclosporine, ushikulides, pentalenolactone I and hygromy$\operatorname{cin} \mathrm{A}$.

47. The method of any one of items 40 to 42 , wherein the secondary metabolite is a flavor such as geosmin.

20

48. The method of any one of items 40 to 42 , wherein the secondary metabolite is a parasiticide such as an insecticide, an anthelmintic, a larvacide, or an antiprotozoal agent such as spinsad or avermectin.

49. The method of any one of items 27 to 39 , wherein the at least one nucleic acid encodes an enzyme such as a metabolic enzyme selected from the group consisting of an amylase, a protease, a cellulase, a chitinase, a keratinase and a xylanase, a glycosyltransferase, an oxygenase, a hydroxylase, a methyltransferase, a dehydrogenase, a dehydratase.

50. The method of any one of items 27 to 49 , wherein the generation of at least one indel results in the inactivation of at least one gene. 
51. A method for selectively modulating transcription of at least one target nucleic acid sequence in a host cell, the method comprising introducing into the host cell:

i. at least one guiding means, or a nucleic acid comprising a nucleotide sequence encoding guiding means, wherein the guiding means comprises a nucleotide sequence that is complementary to a target nucleic acid sequence in the host cell; and

ii. a variant Cas9, or a nucleic acid comprising a nucleotide sequence encoding the variant Cas9, wherein the variant Cas 9 has reduced endodeoxyribonuclease activity, wherein said guiding means and said variant Cas9 form a complex in the host cell, said complex selectively modulating transcription of at least one target nucleic acid in the host cell.

52. The method of item 51 , wherein the guiding means comprises at least one sgRNA and/or at least one crRNA/tracrRNA set.

53. The method of item 52 , wherein the variant Cas 9 can cleave one of the strands of the target nucleic acid sequence but has reduced ability to cleave the other strand of the target nucleic acid sequence.

54. The method of any one of items 51 to 53 , wherein the variant Cas 9 is selected from the group consisting of Cas9-H840A, Cas9-D10A and Cas9H840A,D10A.

25

55. The method of any one of items 51 to 54 , wherein the host cell is a prokaryotic cell selected from the group consisting of actinobacteria.

56. The method of any one of items 51 to 55 , wherein the host cell is selected from the group consisting of Actinomycetales, such as Streptomyces sp., Amycolatopsis sp. or Saccharopolyspora sp.

57. The method of any one of items 51 to 56 , wherein the host cell is selected from the group consisting of Streptomyces coelicolor, Streptomyces avermitilis, Streptomyces aureofaciens, Streptomyces griseus, Streptomyces 
parvulus, Streptomyces albus, Streptomyces vinaceus, Streptomyces acrimycinis, Streptomyces calvuligerus, Streptomyces lividans, Streptomyces limosus, Streptomyces rubiqinosis, Streptomyces azureus, Streptomyces glaucenscens, Streptomyces rimosus, Streptomyces violaceoruber, Streptomyces kanamyceticus, Amycolatopsis orientalis, Amycolatopsis mediterranei and Saccharopolyspora erythraea.

58. The method of any one items 51 to 57 , wherein the at least one target nucleic acid sequence is comprised within a secondary metabolite biosynthetic gene.

59. The method of any one items 51 to 58 , wherein the at least one target nucleic acid sequence is comprised within a gene cluster such as a secondary metabolite gene cluster.

60. The method of any one items 58 to 59 , wherein the secondary metabolite is selected from the group consisting of antibiotics, herbicides, anti-cancer agents, immunosuppressants, flavors, parasiticides, enzymes and proteins.

61. The method of any one items 58 to 60 , wherein the secondary metabolite is an antibiotic selected from the group consisting of apramycin, bacitracin, chloramphenicol cephalosporins, cycloserine, erythromycin, fosfomycin, gentamicin, kanamycin, kirromycin, lassomycin, lincomycin, lysolipin, microbisporicin, neomycin, noviobiocin, nystatin, nitrofurantoin, platensimycin, pristinamycins, rifamycin, streptomycin, teicoplanin, tetracycline, tinidazole, ribostamycin, daptomycin, vancomycin, viomycin, virginiamycin.

62. The method of any one items 58 to 60 , wherein the secondary metabolite is a herbicide selected from the group consisting of bialaphos, resormycin and phosphinothricin.

63. The method of any one items 58 to 60 , wherein the secondary metabolite is an anti-cancer agent selected from the group consisting of doxorubicin, salinosporamides, aclarubicin, pentostatin, peplomycin, thrazarine and neocarcinostatin. 
64. The method of any one items 58 to 60 , wherein the secondary metabolite is an immunosuppressant selected from the group consisting of rapamycin, FK520, FK506, cyclosporine, ushikulides, pentalenolactone I and hygromy$\operatorname{cin} \mathrm{A}$.

65. The method of any one items 58 to 60 , wherein the secondary metabolite is a flavor such as geosmin.

66. The method of any one items 58 to 60 , wherein the secondary metabolite is a parasiticide such as an insecticide, an anthelmintic, a larvacide, or an antiprotozoal agent such as spinsad or avermectin.

67. The method of any one items 51 to 57 , wherein the at least one nucleic acid encodes an enzyme such as a metabolic enzyme selected from the group consisting of an amylase, a protease, a cellulase, a chitinase, a keratinase and a xylanase, a glycosyltransferase, an oxygenase, a hydroxylase, a methyltransferase, a dehydrogenase, a dehydratase.

68. The method of any one of items 51 to 67 , wherein:

i. the transcription of the guiding means is under the control of an inducible promoter; or

ii. the expression of the variant Cas 9 is inducible.

69. A polynucleotide having at least $93 \%$ identity with SEQ ID NO: 1 , such as at least $94 \%$ identity, such as at least $95 \%$ identity, such as at least $96 \%$ identity, such as at least $97 \%$ identity, such as at least $98 \%$ identity, such as at least $99 \%$ identity, such as $100 \%$ identity.

70. The polynucleotide of item 69 , wherein the polynucleotide is non-naturally occurring.

71. A polypeptide encoded by the polynucleotide of any of items 69 to 70 . 
72. The polypeptide of any item 71 , wherein the polypeptide is non-naturally occurring.

73. A cell comprising the polynucleotide of any of items 69 to 70 .

5

74. A cell comprising the polypeptide of any of items 71 to 72 .

75. A vector comprising the polynucleotide of any of items 69 to 70.

10

15

20

25
76. A clonal library obtainable by the method of any of items 1 to 26 , said clonal library comprising a plurality of clones, each clone harbouring at least one deletion around at least one target nucleic acid sequence, wherein each of said deletion is a deletion of at least $1 \mathrm{bp}$.

77. A kit for performing the method of any of items 1 to 26 , said kit comprising: - a vector comprising a nucleic acid sequence encoding a Cas9 nuclease or variant thereof; and

- instructions for use.

78. The kit of item 77 , wherein the nucleic acid sequence is the polynucleotide of items 69 to 70 .

79. The kit of any one of items 77 to 78 , further comprising at least one guiding means and/or at least one host cell.

80. The kit of any one of items 77 to 79 , wherein the host cell has a nonhomologous end-joining (NHEJ) pathway which is at least partly deficient.

81. The kit of any one of items 77 to 80 , further comprising means for partly inactivating NHEJ in the host cell.

82. A kit for performing the method of any of items 27 to 50 , said kit comprising:

- a first vector comprising a nucleic acid sequence encoding Cas9 or a variant thereof; and

- instructions for use. 
83. The kit of item 82, further comprising a second vector comprising at least one nucleic acid encoding at least one of the NHEJ activities defined in item 33.

5

84. The kit of item 83, wherein the at least one nucleic acid encodes a ligase derived from S. carneus.

85. A kit for performing the method of any of items 51 to 68 , said kit comprising:

10 - a vector comprising a nucleic acid sequence encoding a variant Cas9; and

- instructions for use.

86. The kit of item 85, wherein the variant Cas9 is Cas9-H840A, Cas9-D10A or 15 Cas9-H840A,D10A.

87. The kit of any of items 85 to 86 , further comprising at least one guiding means and/or at least one host cell. 


\section{Claims}

1. A method for generating at least one deletion around at least one target nucleic acid sequence comprised within a host cell having a non-homologous end-joining (NHEJ) pathway which is at least partly deficient, said method comprising the steps of:

(i) optionally, restoring the full functionality of the NHEJ pathway,

(ii) inducing a CRISPR-Cas9 system in said host cell, wherein said CRISPR-Cas9 system is able to generate at least one break in said at least one target nucleic acid sequence and wherein the CRISPRCas9 system comprises a Cas9 nuclease and at least one guiding means,

thereby generating:

a. if the method does not comprise step (i)., at least one random-sized deletion around said at least one target nucleic acid sequence, wherein said at least one deletion is a random-sized deletion of at least $1 \mathrm{bp}$; or

b. if the method does comprise step (i), at least one indel around said at least one target nucleic acid sequence, wherein said at least one indel is a deletion or insertion of at least1 bp.

2. The method of claim 1, wherein the host cell is an actinobacterium, such as an Actinomycetales, such as Streptomyces sp., Amycolatopsis sp. or Saccharopolyspora $s p$, such as wherein the host cell is selected from the group consisting of Streptomyces coelicolor, Streptomyces avermitilis, Streptomyces aureofaciens, Streptomyces griseus, Streptomyces parvulus, Streptomyces albus, Streptomyces vinaceus, Streptomyces acrimycinis, Streptomyces calvuligerus, Streptomyces lividans, Streptomyces limosus, Streptomyces rubiqinosis, Streptomyces azureus, Streptomyces glaucenscens, Streptomyces rimosus, Streptomyces violaceoruber, Streptomyces kanamyceticus, Amycolatopsis orientalis, Amycolatopsis mediterranei and Saccharopolyspora erythraea. least $95 \%$ identity, such as at least $96 \%$ identity, such as at least $97 \%$ iden- 
tity, such as at least $98 \%$ identity, such as at least $99 \%$ identity, such as $100 \%$ identity, said polynucleotide encoding a Cas9 nuclease or a variant thereof.

4. The polynucleotide of claim 3, wherein the Cas9 nuclease or variant thereof is codon-optimised for Streptomycetes.

5. A polypeptide encoded by the polynucleotide of any one of claims 3 to 4 .

6. A cell comprising the polynucleotide of claims 3 or 4 .

7. A cell comprising the polypeptide of claim 5 .

8. A vector comprising the polynucleotide of claims 3 or 4 .

15

9. A clonal library obtainable by the method of any of claims 1 to 2 , said clonal library comprising a plurality of clones harbouring at least one deletion and/or indel around at least one target nucleic acid sequence, wherein said deletion is a random-sized deletion of at least $1 \mathrm{bp}$ and wherein said indel is a deletion or insertion of at least $1 \mathrm{bp}$.

10. A method for selectively modulating transcription of at least one target nucleic acid sequence in a host cell, the method comprising introducing into the host cell:

(i) at least one guiding means, or a nucleic acid comprising a nucleotide sequence encoding guiding means, wherein the guiding means comprises a nucleotide sequence that is complementary to a target nucleic acid sequence in the host cell; and

(ii) a variant Cas9, or a nucleic acid comprising a nucleotide sequence encoding the variant Cas9, wherein the variant Cas9 is a variant of the polypeptide of claim 5 or of a polypeptide encoded by the nucleotide sequence encoding the variant Cas 9 of claims 3 or 4 , with reduced endodeoxyribonuclease activity and is codon-optimised for Streptomycetes, 
wherein said guiding means and said variant Cas9 form a complex in the host cell, said complex selectively modulating transcription of at least one target nucleic acid in the host cell.

5

10

15

20

25
11. The method of claim 10, wherein the host cell is an actinobacterium, preferably the host cell is selected from the group consisting of Actinomycetales, Streptomyces sp., Amycolatopsis sp. and Saccharopolyspora sp, even more preferably the host cell is selected from the group consisting of Streptomyces coelicolor, Streptomyces avermitilis, Streptomyces aureofaciens, Streptomyces griseus, Streptomyces parvulus, Streptomyces albus, Streptomyces vinaceus, Streptomyces acrimycinis, Streptomyces calvuligerus, Streptomyces lividans, Streptomyces limosus, Streptomyces rubiqinosis, Streptomyces azureus, Streptomyces glaucenscens, Streptomyces rimosus, Streptomyces violaceoruber, Streptomyces kanamyceticus, Amycolatopsis orientalis, Amycolatopsis mediterranei and Saccharopolyspora erythraea.

12. A kit for performing the method of any of claims 1 to 2 , said kit comprising:

- a vector comprising a nucleic acid sequence encoding a Cas9 nuclease or variant thereof; and

- instructions for use.

13. A kit for performing the method of any of claims 10 to 11 , said kit comprising:

- a vector comprising a variant Cas9, or a nucleic acid comprising a nucleotide sequence encoding the variant Cas 9 , wherein the variant Cas 9 is the polypeptide of claim 5 or the nucleotide sequence encoding the variant Cas 9 is the polynucleotide of claims 3 or 4 , and wherein the variant Cas 9 has reduced endodeoxyribonuclease activity; and

- instructions for use. 
$1 / 16$

Figure 1

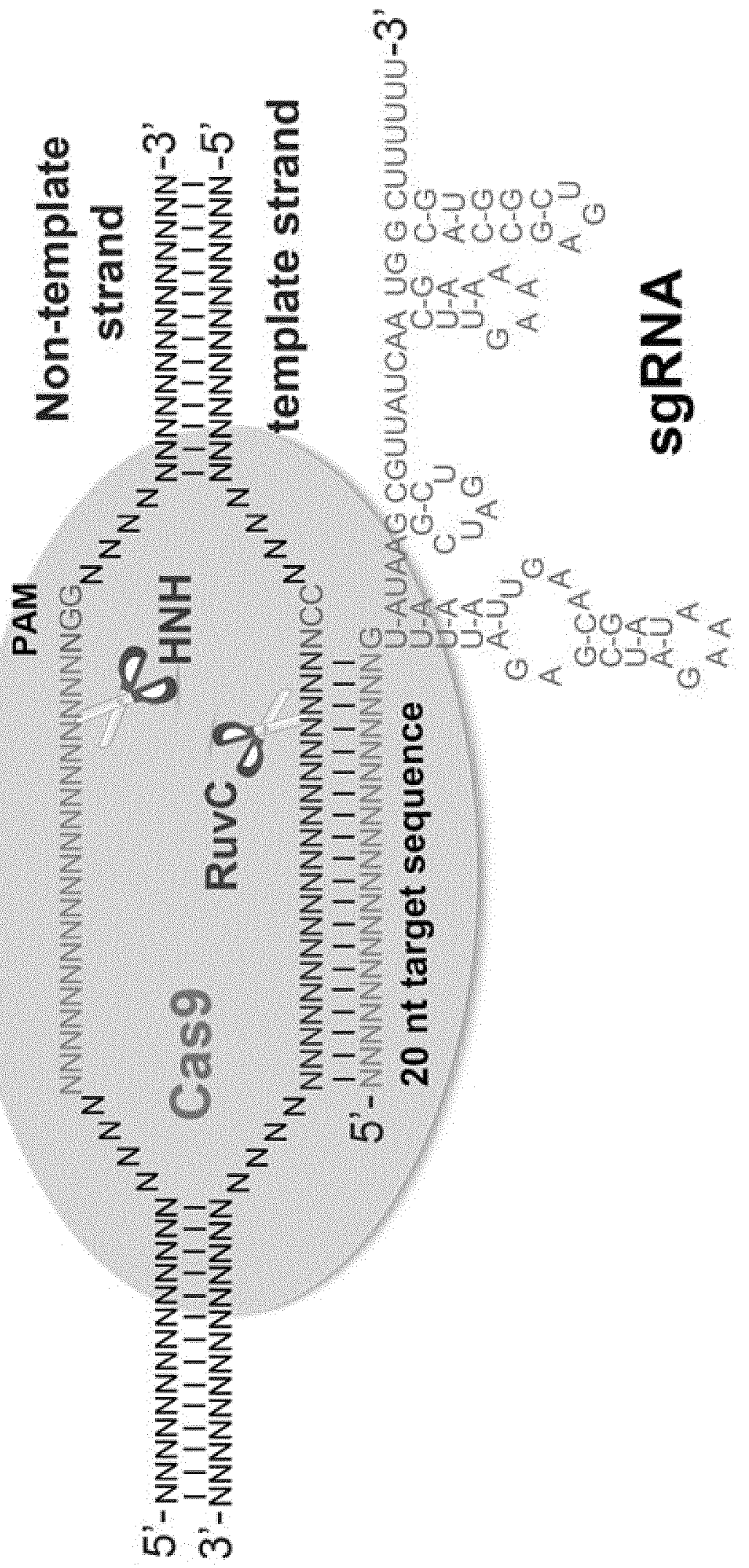


$2 / 16$

Figure 2

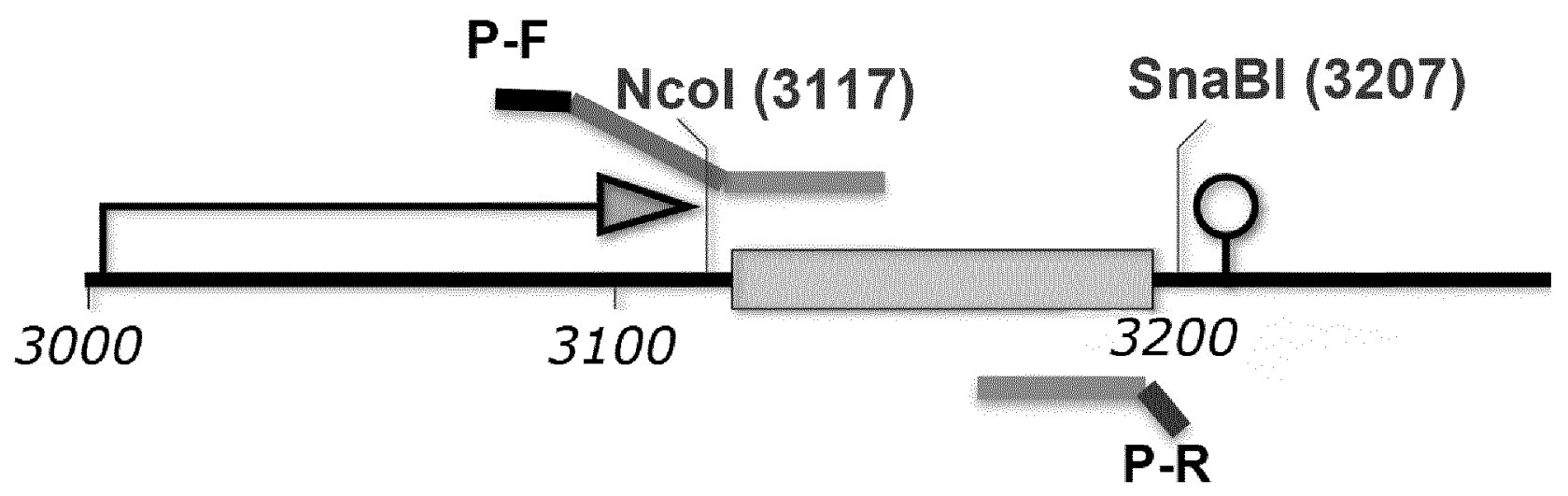


$3 / 16$

Figure 3

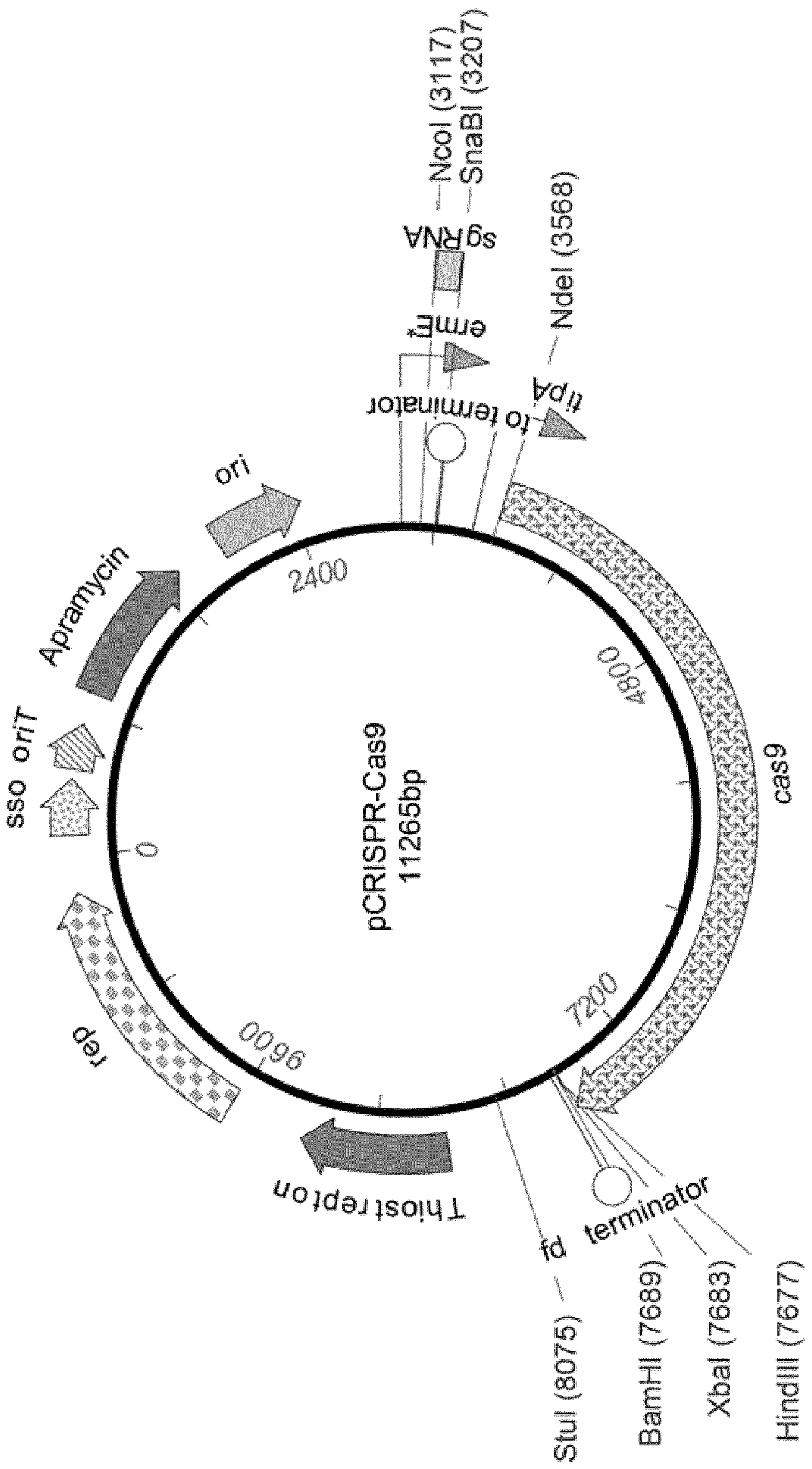


Figure 4
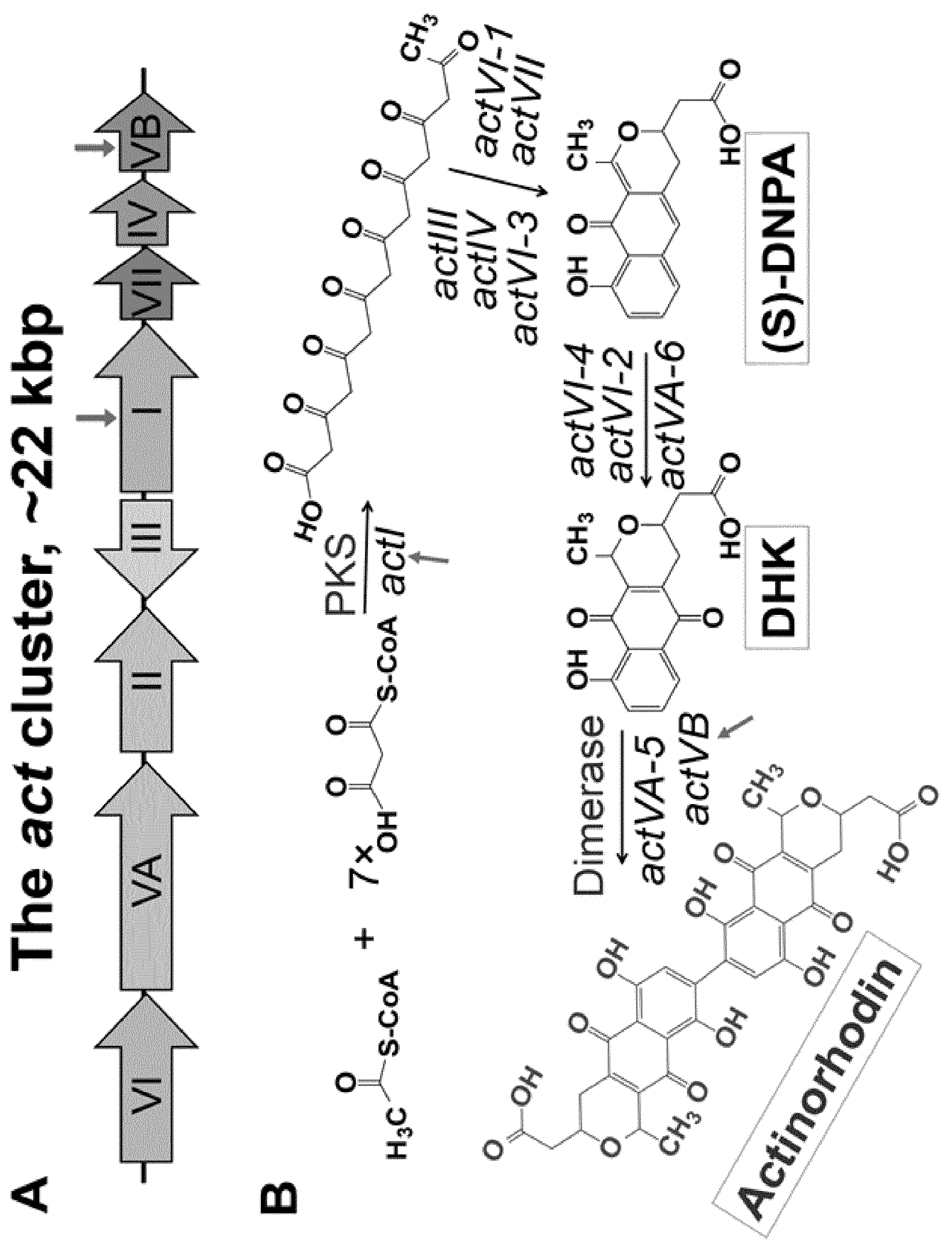
$5 / 16$

Figure 5

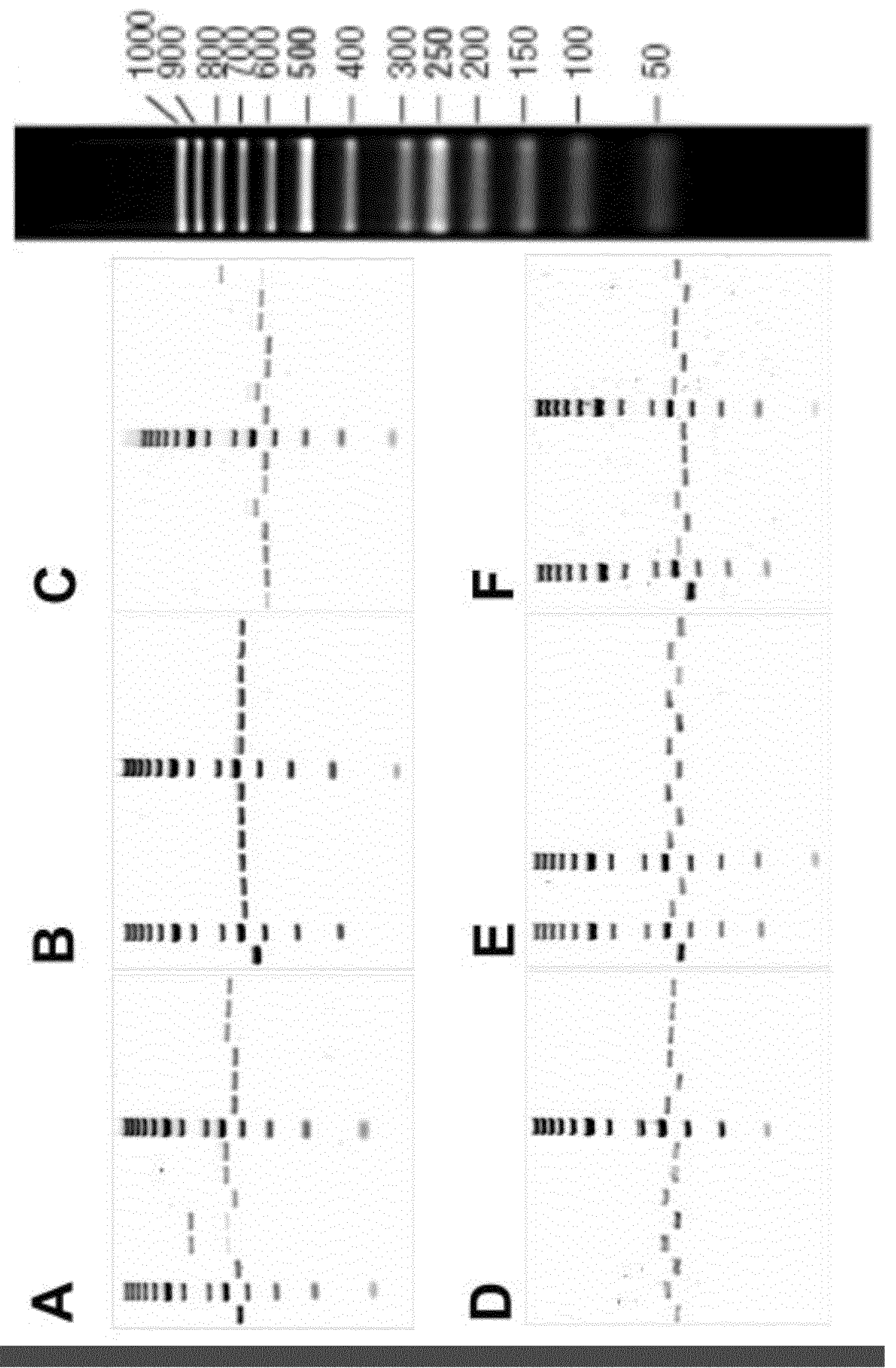


$6 / 16$

Figure 6
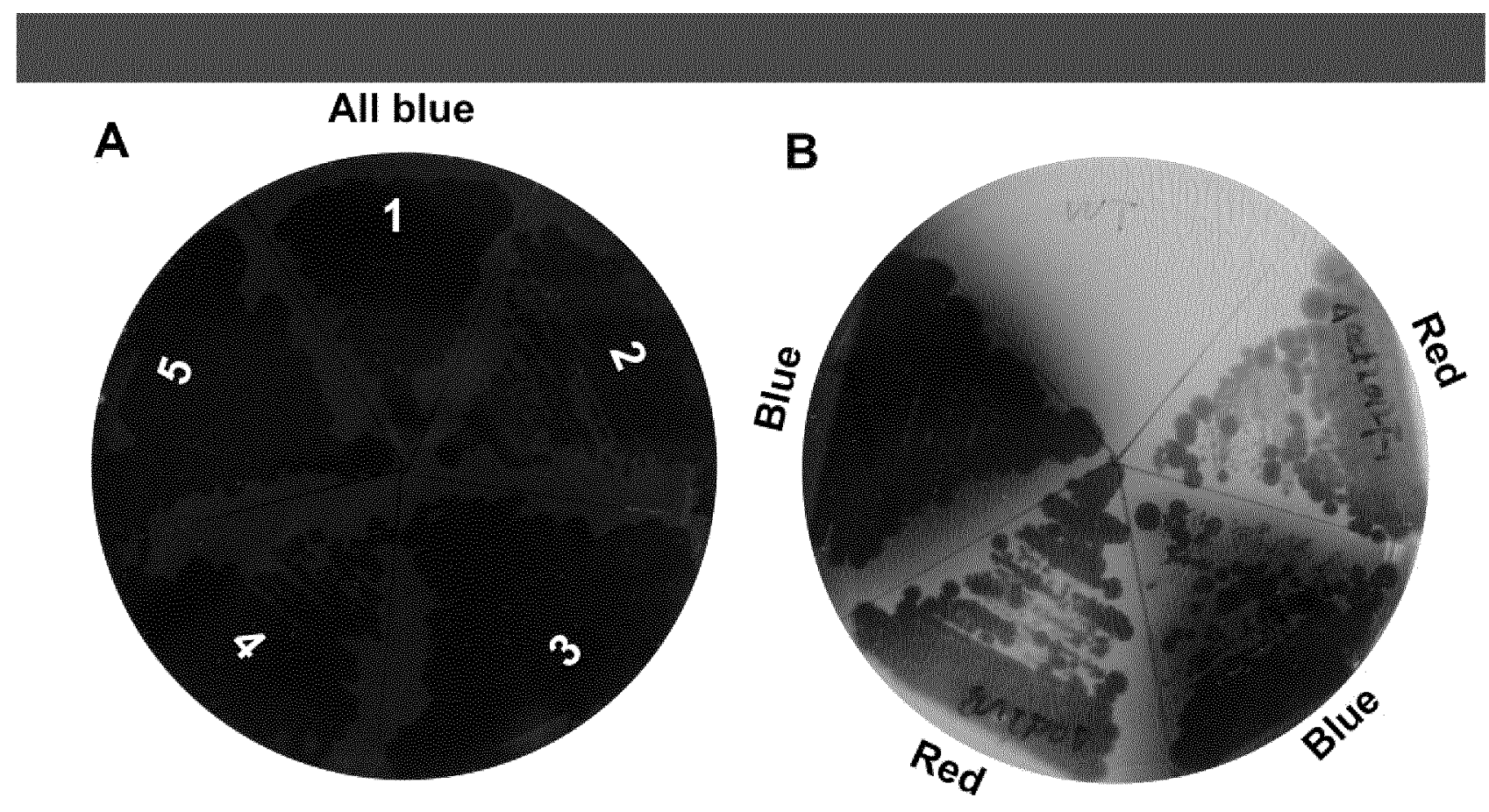
Figure 7

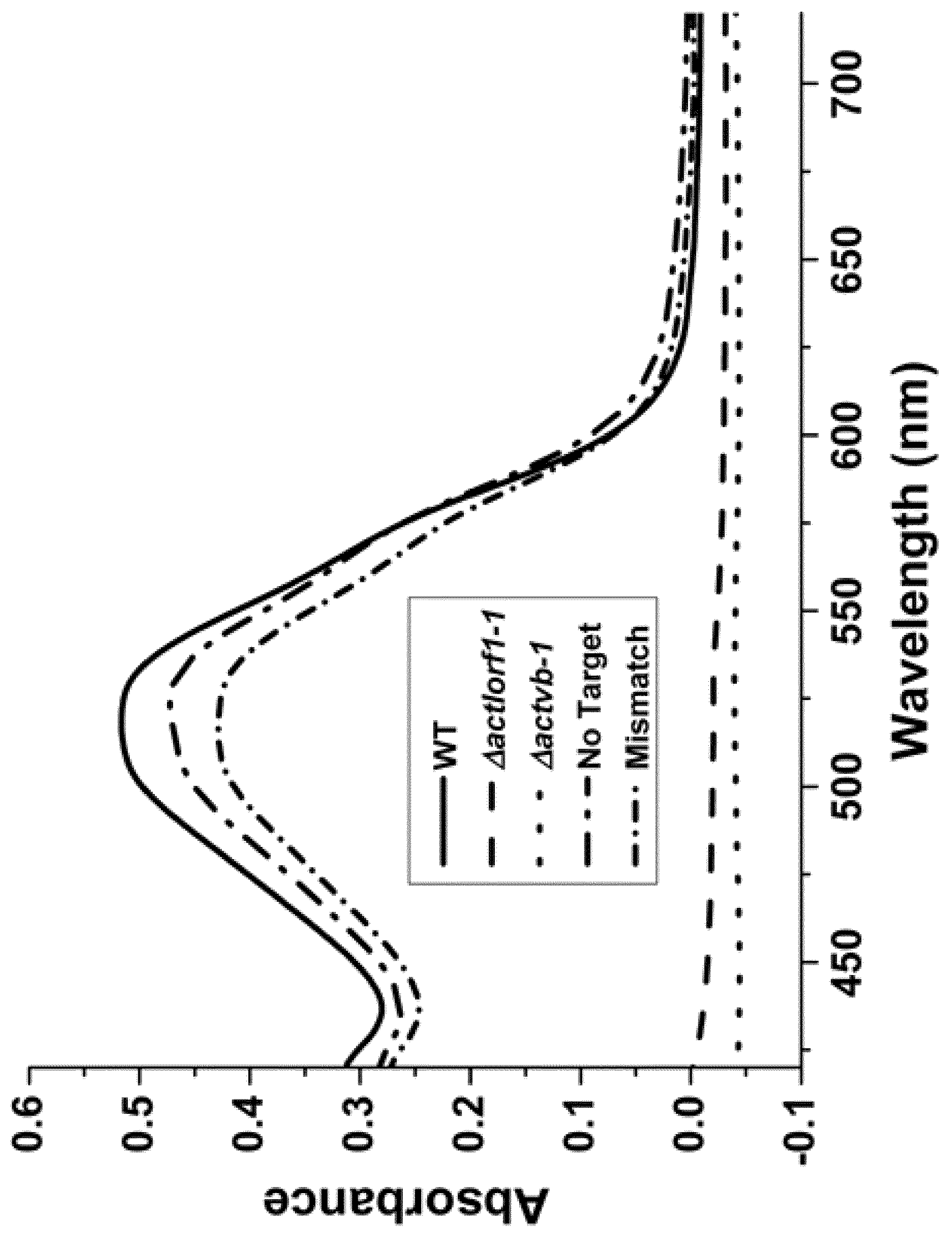


$8 / 16$

Figure 8

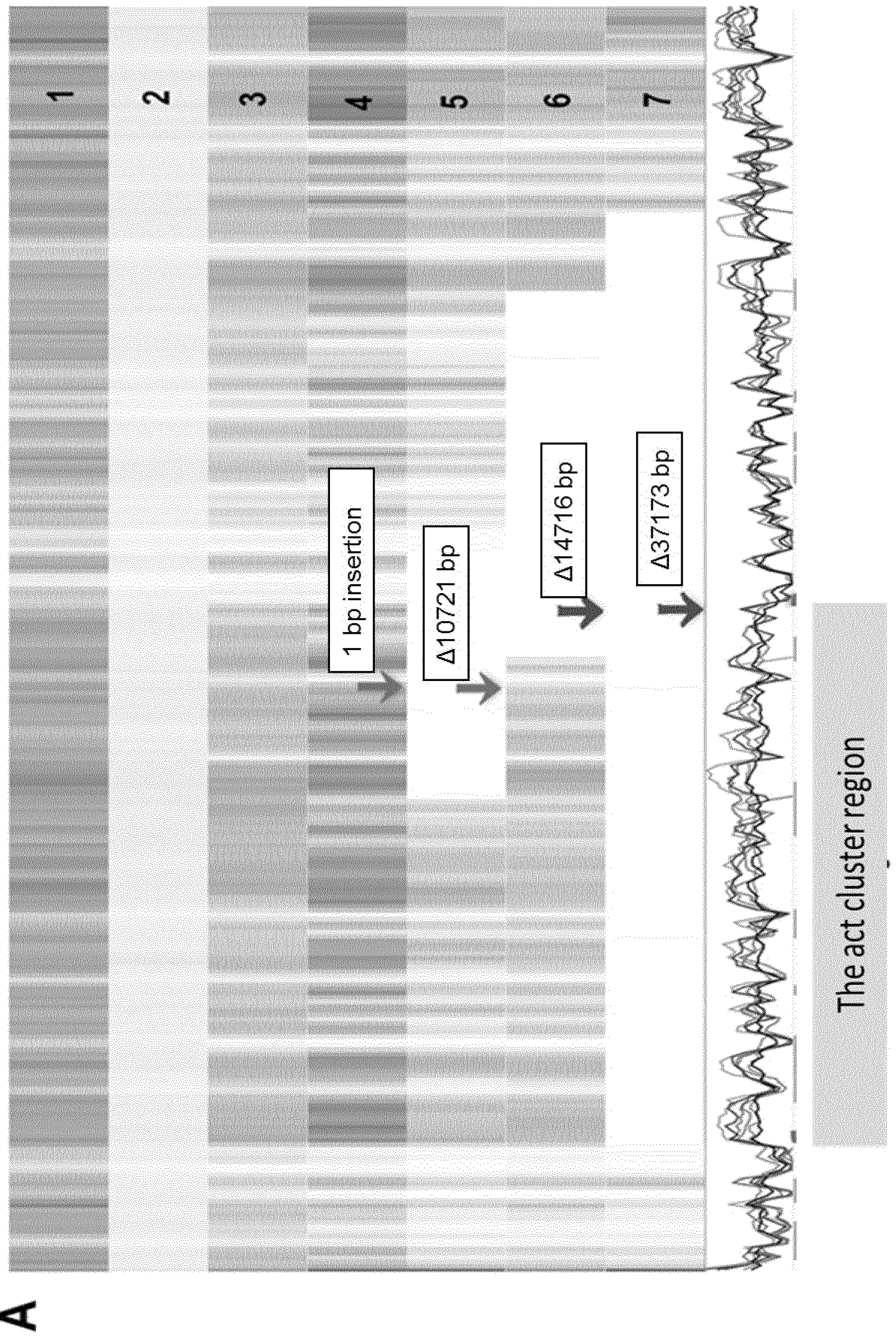


$9 / 16$

Figure 8 (CONT.)

B

sgRNA: Actlorf1-1 NT

1 ATCCGGCATCGAGGGGTCCCGTATCGGCCTTCG--AGCCTCCTTCGAGCCACGGGGCCGA

4 ATCCGGCATCGAGGGGTCCCGTATCGGCCTTCGA-AGCCTCCTTCGAGCCACGGGGCCGA

C sgRNA: Actlorf1-6 T

PAM

1 ACGCGCTGCAACGCGTACCACA-TTGACGGGCCTGAAGGCCGACGGCCGCGAGATGGCCGAGACCATCC Nis:

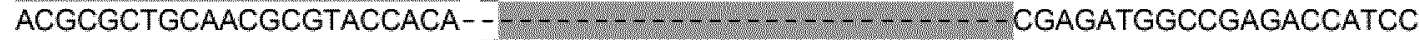
ACGCGCTGCAACGCGTACCACA-IT GACGGGCCTGAAGGCCGACGGCCGCGAGATGGCCGAGACCATCC ACGCGCTGCAACGCGTACCACA-AT GACGGGCCTGAAGGCCGACGGCCGCGAGATGGCCGAGACCATCC ACGCGCTGCAACGCGTACCACAA TGACGGGCCTGAAGGCCGACGGCCGCGAGATGGCCGAGACCATCC ACGCGCTGCAACGCGTACCACATATGACGGGCCTGAAGGCCGACGGCCGCGAGATGGCCGAGACCATCC ACGCGCTGCAACGCGTACCACA - TTACG GGCCTGAAGGCCGACGGCCGCGAGATGGCCGAGACCATCC ACGCGCTGCAACGCGTACCACAAATGACGGGCCTGAAGGCCGACGGCCGCGAGATGGCCGAGACCATCC

sgRNA: Actvb-2 NT

D

$$
\text { PAM }
$$

1 EGAGCTGGTCGCCTACAACGGTGGGCAGCTGCCCACCTGCCTCGCCTAGGGAGTICGAAGATGGCAGCC GGAGCTGGTCGCCTACAACGGTGGGCAGCTGCCCACCTGCCTCGCCTAGG - - TTCGAAGATGGCAGCC GGAGCTGGTCGCCTACAACGGTGGGCAGCTGCCCACCTGCCTCGCCTAGGGAGTTCGA GGATGGCAGCC GGAGCTGGTCGCCTACAACGGTGGGCAGCTGCCCACCTGCCTCGCCT GGGGATTCGAAGATGGCAGCO GGAGCTGGTCGCCTACAACGGTGGGCAGCTGCCCACCTGCCTCGCCTAGGG GTTCGAAGATGGCAGCC GGAGCTGGTCGCCTACAACGGTGGGCAGCTGCCCACCTGCCTCGCOT IGGGAGTTCGAAGATGGCAGCC GGAGCTGGTCGCCTACAACGGTGGGCAGCTGCCCACCTGCCTCGCC GGGGAGTTCGAAGATGGCAGCC GGAGCTGGTCGCCTACAACGGTGGGCAGCTGCCCACCTGCCTCGCCT OGGGAGTTCGAAGATGGCAGCC

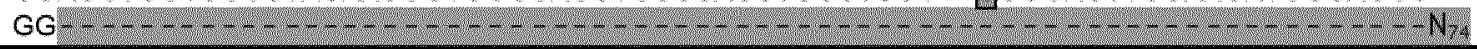




\section{$10 / 16$}

Figure 9

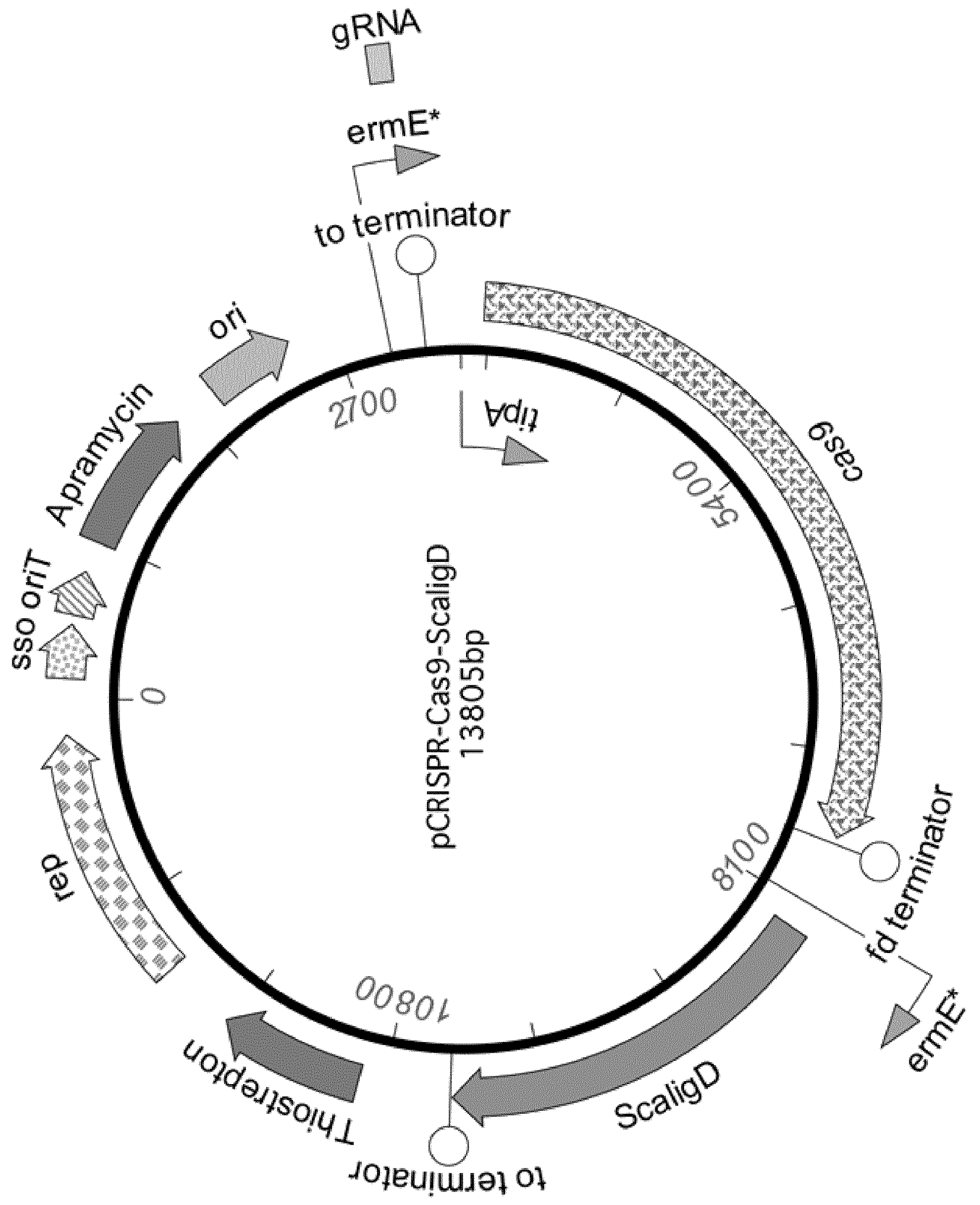


Figure 10
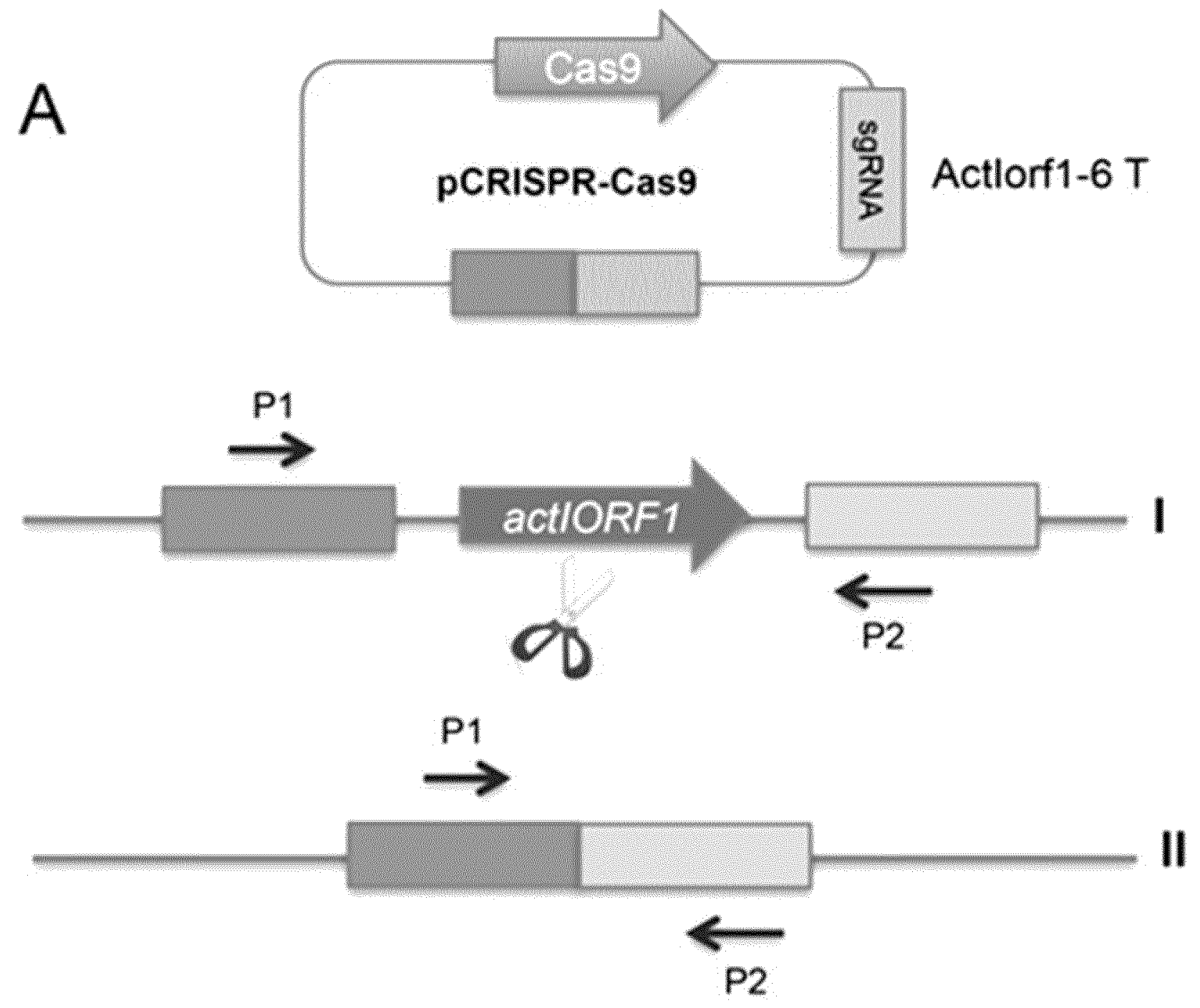
$12 / 16$

Figure 10 (CONT.)
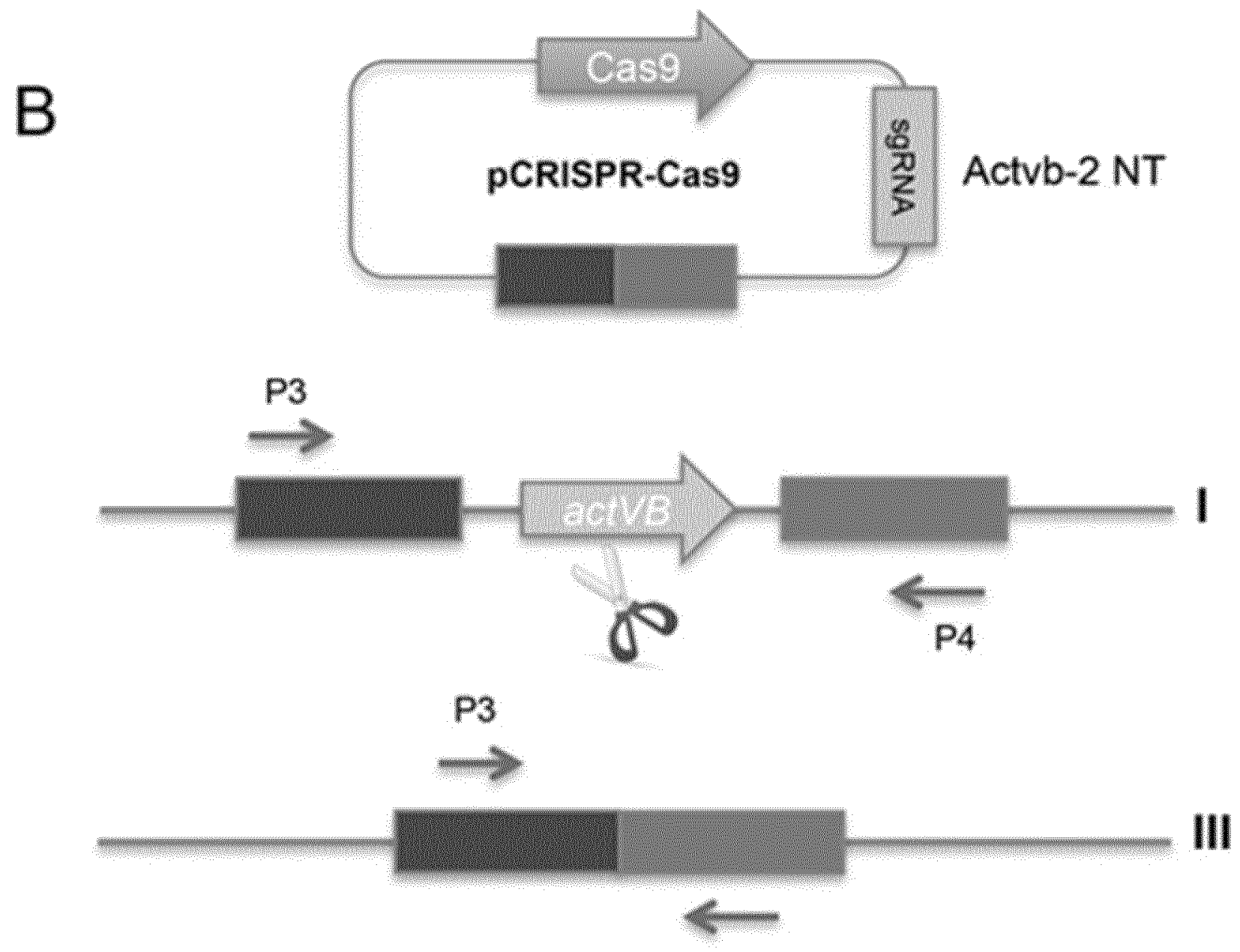
$13 / 16$

Figure 10 (CONT.)

C

$\begin{array}{lllllllllll}\text { WT } & 1 & 2 & 3 & 4 & 5 & 6 & 7 & 8 & 9 & 10\end{array}$
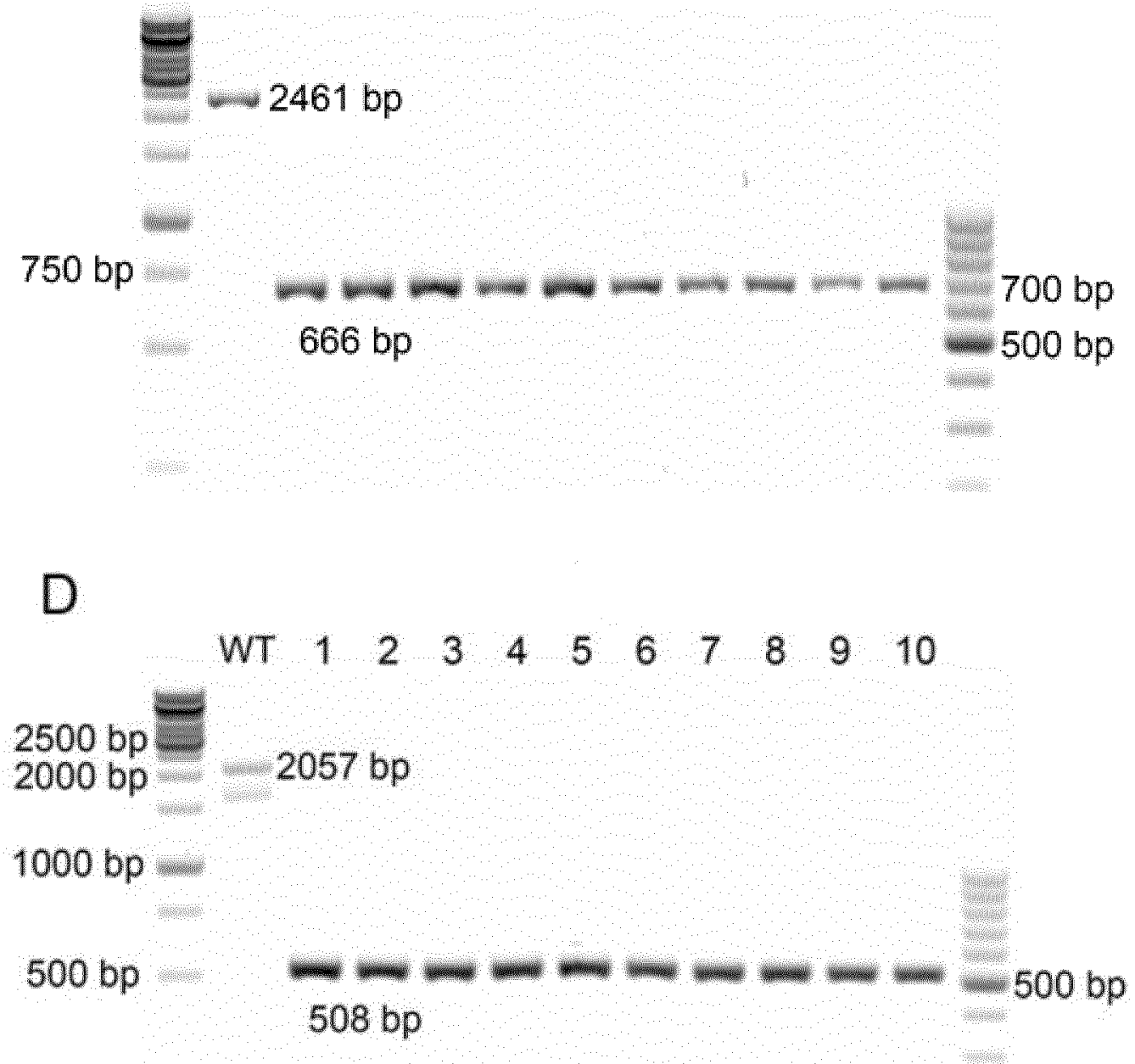
Figure 11

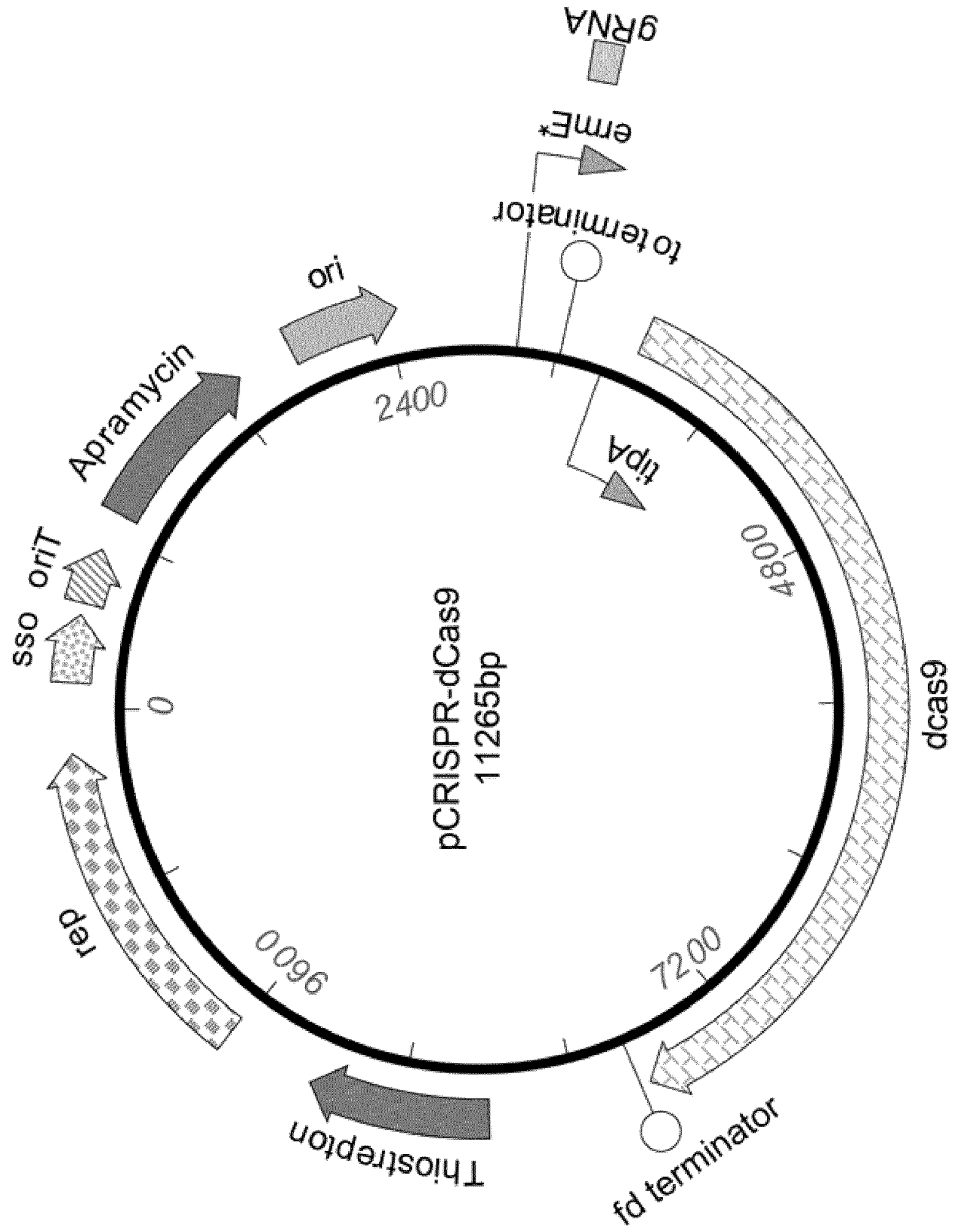


Figure 12

A

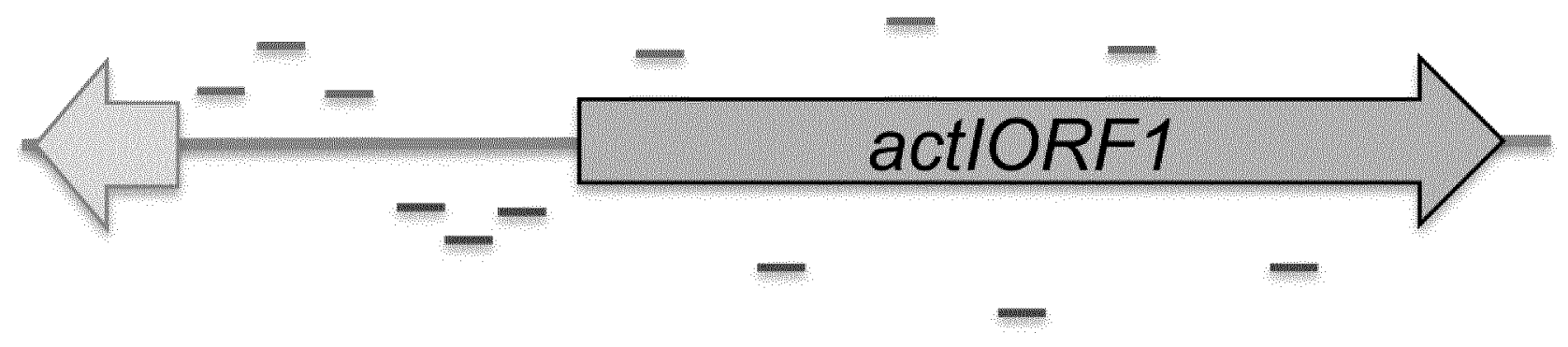

B

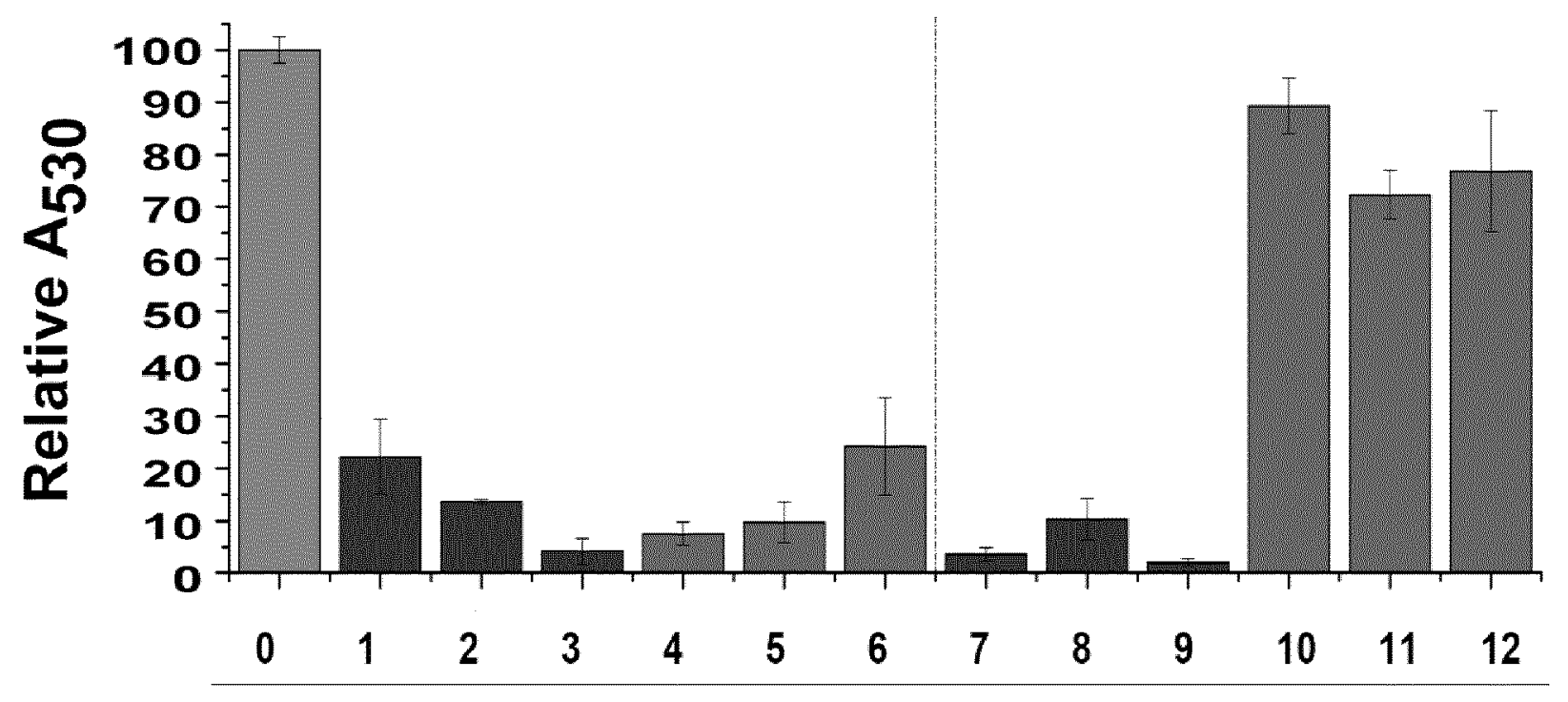


Figure 12 (CONT.)

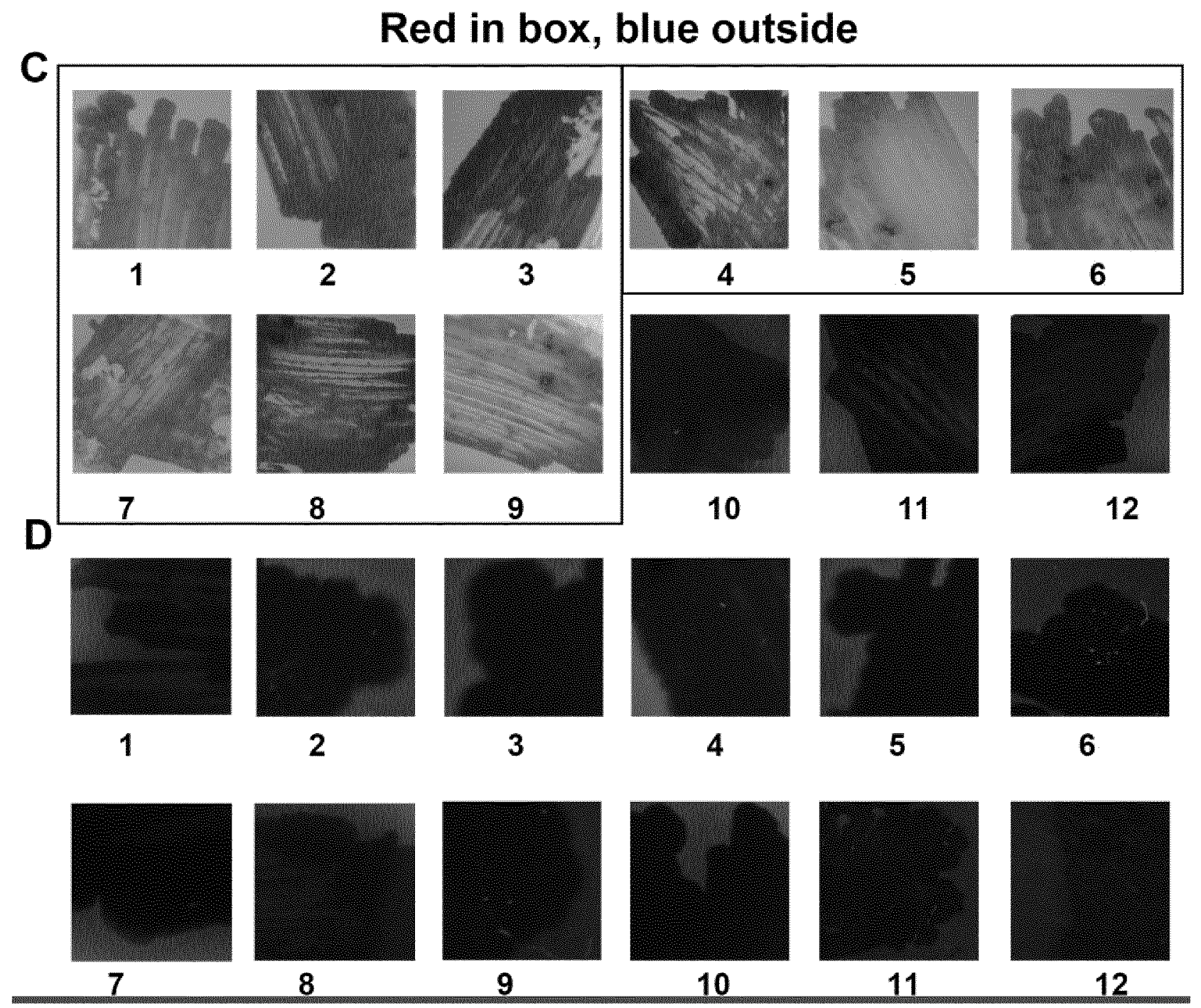


Box No. II Observations where certain claims were found unsearchable (Continuation of item 2 of first sheet)

This international search report has not been established in respect of certain claims under Article 17(2)(a) for the following reasons:

1. $\square$ Claims Nos.:

because they relate to subject matter not required to be searched by this Authority, namely:

2. Claims Nos:

because they relate to parts of the international application that do not comply with the prescribed requirements to such an extent that no meaningful international search can be carried out, specifically:

3

Claims Nos.

because they are dependent claims and are not drafted in accordance with the second and third sentences of Rule 6.4(a).

Box No. III Observations where unity of invention is lacking (Continuation of item 3 of first sheet)

This International Searching Authority found multiple inventions in this international application, as follows:

\section{see additional sheet}

1. As all required additional search fees were timely paid by the applicant, this international search report covers all searchable claims.

2. As all searchable claims could be searched without effort justifying an additional fees, this Authority did not invite payment of additional fees.

3. As only some of the required additional search fees were timely paid by the applicant, this international search report covers only those claims for which fees were paid, specifically claims Nos.:

4. $\mathrm{x}$ No required additional search fees were timely paid by the applicant. Consequently, this international search report is restricted to the invention first mentioned in the claims; it is covered by claims Nos.:

$1,2,9,12$ (all partially)

Remark on Protest

The additional search fees were accompanied by the applicant's protest and, where applicable, the payment of a protest fee.

The additional search fees were accompanied by the applicant's protest but the applicable protest fee was not paid within the time limit specified in the invitation.

No protest accompanied the payment of additional search fees. 


\begin{tabular}{l} 
A. CLASSIFICATIONOF SUBJECT MATTER \\
INV. C12N15/63 CI2N $15 / 90$ \\
ADD. \\
According to International Patent Classification (IPC) or to both national classification and IPC \\
B. FIELDS SEARCHED \\
\hline Minimum documentation searched (classification system followed by classification symbols) \\
C12N
\end{tabular}

Documentation searched other than minimum documentation to the extent that such documents are included in the fields searched

Electronic data base consulted during the international search (name of data base and, where practicable, search terms used)

EP0-Internal, WPI Data, BIOSIS, CHEM ABS Data, Sequence Search, EMBASE

\section{DOCUMENTS CONSIDERED TO BE RELEVANT}

\begin{tabular}{|c|c|c|}
\hline Category $^{*}$ & Citation of document, with indication, where appropriate, of the relevant passages & Relevant to claim No. \\
\hline$\gamma$ & $\begin{array}{l}\text { H. HUANG ET AL: "One-step high-efficiency } \\
\text { CRISPR/Cas9-mediated genome editing in } \\
\text { Streptomyces", } \\
\text { ACTA BIOCHIMICA ET BIOPHYSICA SINICA, } \\
\text { vol. } 47, \text { no. } 4,3 \text { March } 2015 \text { (2015-03-03), } \\
\text { pages } 231-243, \text { XP055204421, } \\
\text { ISSN: 1672-9145, D0I: 10.1093/abbs/gmv007 } \\
\text { page 235, column 1, paragraph } 1 \text { - page } \\
\text { 238, column 1, paragraph } 1 \\
----\end{array}$ & $1,2,9,12$ \\
\hline
\end{tabular}

$X$ Further documents are listed in the continuation of Box $C . \quad X$ See patent family annex.

Special categories of cited documents

" $A$ " document defining the general state of the art which is not considered to be of particular relevance

"E" earlier application or patent but published on or after the international filing date

"L" document which may throw doubts on priority claim(s) or which is cited to establish the publication date of another citation or other

"O" document referring to an oral disclosure, use, exhibition or other means

"P" document published prior to the international filing date but later than the priority date claimed

Date of the actual completion of the international search

13 May 2016 special reason (as specified)

"T" later document published after the international filing date or priority date and not in conflict with the application but cited to understand the principle or theory underlying the invention

"X" document of particular relevance; the claimed invention cannot be considered novel or cannot be considered to involve an inventive step when the document is taken alone

" $Y$ " document of particular relevance; the claimed invention cannot be considered to involve an inventive step when the document is
combined with one or more other such documents, such combination being obvious to a person skilled in the art

"\&" document member of the same patent family

Date of mailing of the international search report

$03 / 08 / 2016$

Name and mailing address of the ISA/

European Patent Office, P.B. 5818 Patentlaan 2

NL - 2280 HV Rijswijk

Tel. $(+31-70) 340-2040$

Fax: $(+31-70)$ 340-3016

Burkhardt, Peter 
INTERNATIONAL SEARCH REPORT

\begin{tabular}{|c|c|c|}
\hline Category ${ }^{*}$ & Citation of document, with indication, where appropriate, of the relevant passages & Relevant to claim No. \\
\hline$Y$ & $\begin{array}{l}\text { XIAOJUAN ZHANG ET AL: "Deletion of } \\
\text { homologs increases gene targeting } \\
\text { frequency in", } \\
\text { JOURNAL OF INDUSTRIAL MICROBIOLOGY \& } \\
\text { BIOTECHNOLOGY ; OFFICIAL JOURNAL OF THE } \\
\text { SOCIETY FOR INDUSTRIAL MICROBIOLOGY, } \\
\text { SPRINGER, BERLIN, DE, } \\
\text { vo1. 39, no. 6, } \\
21 \text { February } 2012 \text { (2012-02-21), pages } \\
917-925, \text { XPO35060031, } \\
\text { ISSN: 1476-5535, DOI: } \\
\text { 10.1007/S10295-012-1097-X } \\
\text { page } 919, \text { column 2, paragraph } 3 \text { - page } \\
923, \text { column 2, paragraph } 1\end{array}$ & $1,2,9,12$ \\
\hline$Y$ & $\begin{array}{l}\text { US 2015/079680 A1 (BRADLEY ALLAN [GB] ET } \\
\text { AL) } 19 \text { March } 2015 \text { (2015-03-19) } \\
\text { paragraph [0010] - paragraph [0011] }\end{array}$ & $1,2,9,12$ \\
\hline$A$ & $\begin{array}{l}\text { THERESA SIEGL ET AL: "I-SCeI } \\
\text { endonuclease: a new tool for DNA repair } \\
\text { studies and genetic manipulations in } \\
\text { streptomycetes", } \\
\text { APPLIED MICROBIOLOGY AND BIOTECHNOLOGY, } \\
\text { SPRINGER, BERLIN, DE, } \\
\text { vo1. } 87, \text { no. 4, 15 May } 2010 \text { (2010-05-15), } \\
\text { pages 1525-1532, XPO19841641, } \\
\text { ISSN: 1432-0614 } \\
\text { page 1531, column 2, paragraph } 1\end{array}$ & $1,2,9,12$ \\
\hline A & $\begin{array}{l}\text { W0 2014/065596 A1 (TOOLGEN INC [KR]) } \\
1 \text { May } 2014(2014-05-01) \\
\text { paragraph [0166]; claims; sequences } 1,108 \\
\text { \& DATABASE Geneseq [Online] }\end{array}$ & $1,2,9,12$ \\
\hline & $\begin{array}{l}3 \text { July } 2014 \text { (2014-07-03), } \\
\text { "Streptococcus pyogens DNA encoding the } \\
\text { Cas9 protein, SEQ 1.", } \\
\text { retrieved from EBI accession no. } \\
\text { GSN:BBF83954 } \\
\text { Database accession no. BBF83954 } \\
\text { sequence } \\
\text { \& DATABASE Geneseq [0nline] }\end{array}$ & \\
\hline & $\begin{array}{l}3 \text { July } 2014 \text { (2014-07-03), } \\
\text { "Streptococcus pyogens DNA encoding the } \\
\text { Cas9 protein, SEQ 108.", } \\
\text { retrieved from EBI accession no. } \\
\text { GSN:BBF84061 } \\
\text { Database accession no. BBF84061 } \\
\text { sequence }\end{array}$ & \\
\hline A & $\begin{array}{l}\text { W0 } 2015 / 013583 \text { A2 (HARVARD COLLEGE [US]) } \\
29 \text { January } 2015(2015-01-29) \\
\text { sequence } 22\end{array}$ & $1,2,9,12$ \\
\hline
\end{tabular}


INTERNATIONAL SEARCH REPORT

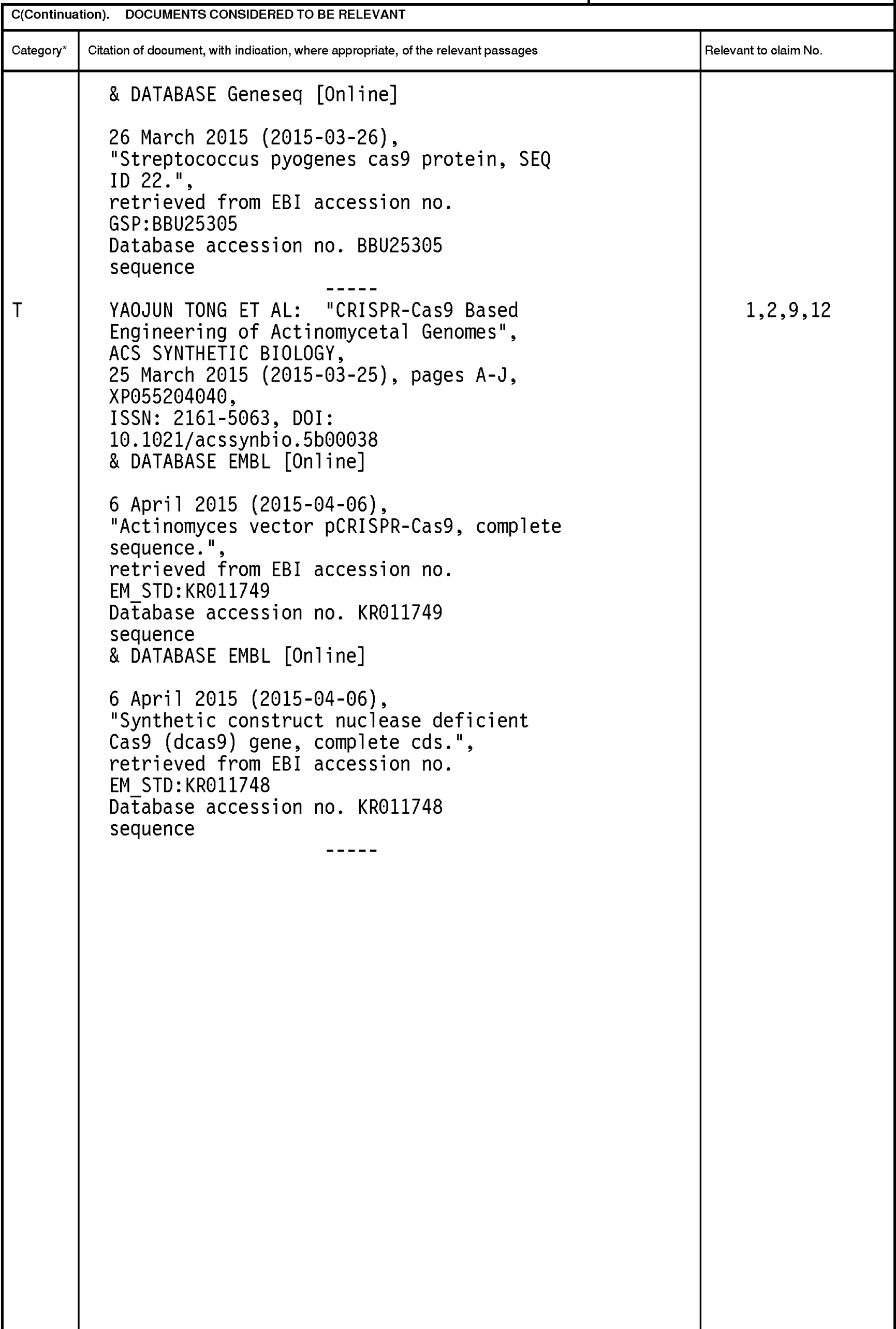


INTERNATIONAL SEARCH REPORT

Information on patent family members

\begin{tabular}{|c|c|c|c|c|}
\hline $\begin{array}{l}\text { Patent document } \\
\text { cited in search report }\end{array}$ & & $\begin{array}{l}\text { Publication } \\
\text { date }\end{array}$ & $\begin{array}{l}\text { Patent family } \\
\text { member(s) }\end{array}$ & $\begin{array}{l}\text { Publication } \\
\text { date }\end{array}$ \\
\hline US 2015079680 & A1 & $19-03-2015$ & $\begin{array}{lrl}\text { CN } & 105637087 & \mathrm{~A} \\
\text { DE } & 202014010413 & \mathrm{U} 1 \\
\text { EP } & 2877571 & \mathrm{~A} 1 \\
\text { TW } & 201542816 & \mathrm{~A} \\
\text { US } & 2015079680 & \mathrm{~A} 1 \\
\text { US } & 2016177340 & \mathrm{~A} 1 \\
\text { US } & 2016207983 & \mathrm{~A} 1 \\
\text { WO } & 2015040402 & \mathrm{~A} 1\end{array}$ & $\begin{array}{l}01-06-2016 \\
08-12-2015 \\
03-06-2015 \\
16-11-2015 \\
19-03-2015 \\
23-06-2016 \\
21-07-2016 \\
26-03-2015\end{array}$ \\
\hline WO 2014065596 & A1 & $01-05-2014$ & $\begin{array}{lrl}\text { AU } & 2013335451 & \mathrm{~A} 1 \\
\mathrm{AU} & 2015218519 & \mathrm{~A} 1 \\
\mathrm{CA} & 2888190 & \mathrm{~A} 1 \\
\mathrm{CN} & 104968784 & \mathrm{~A} \\
\mathrm{CN} & 105441440 & \mathrm{~A} \\
\mathrm{EP} & 2912175 & \mathrm{~A} 1 \\
\mathrm{HK} & 1212732 & \mathrm{~A} 1 \\
\mathrm{JP} & 2016027807 & \mathrm{~A} \\
\mathrm{JP} & 2016500003 & \mathrm{~A} \\
\mathrm{KR} & 20150101446 & \mathrm{~A} \\
\mathrm{KR} & 20150101476 & \mathrm{~A} \\
\mathrm{KR} & 20150101477 & \mathrm{~A} \\
\mathrm{KR} & 20150101478 & \mathrm{~A} \\
\mathrm{SG} & 11201503059 \times & \mathrm{A} \\
\text { US } & 2015284727 & \mathrm{~A} 1 \\
\text { US } & 2015322457 & \mathrm{~A} 1 \\
\text { US } & 2015344912 & \mathrm{~A} 1 \\
\text { WO } & 2014065596 & \mathrm{~A} 1\end{array}$ & $\begin{array}{l}07-05-2015 \\
17-09-2015 \\
01-05-2014 \\
07-10-2015 \\
30-03-2016 \\
02-09-2015 \\
17-06-2016 \\
25-02-2016 \\
07-01-2016 \\
03-09-2015 \\
03-09-2015 \\
03-09-2015 \\
03-09-2015 \\
29-06-2015 \\
08-10-2015 \\
12-11-2015 \\
03-12-2015 \\
01-05-2014\end{array}$ \\
\hline WO 2015013583 & $A 2$ & $29-01-2015$ & $\begin{array}{lrl}\text { AU } & 2014293015 & \mathrm{~A} 1 \\
\mathrm{CA} & 2918540 & \mathrm{~A} 1 \\
\mathrm{CN} & 105473773 & \mathrm{~A} \\
\mathrm{EP} & 3024964 & \mathrm{~A} 2 \\
\mathrm{KR} & 20160035017 & \mathrm{~A} \\
\text { SG } & 112016002170 & \mathrm{~A} \\
\text { US } & 2015031132 & \mathrm{~A} 1 \\
\text { US } & 2015031133 & \mathrm{~A} 1 \\
\text { WO } & 2015013583 & \mathrm{~A} 2\end{array}$ & $\begin{array}{l}11-02-2016 \\
29-01-2015 \\
06-04-2016 \\
01-06-2016 \\
30-03-2016 \\
26-02-2016 \\
29-01-2015 \\
29-01-2015 \\
29-01-2015\end{array}$ \\
\hline \multicolumn{5}{|c|}{ ' } \\
\hline
\end{tabular}




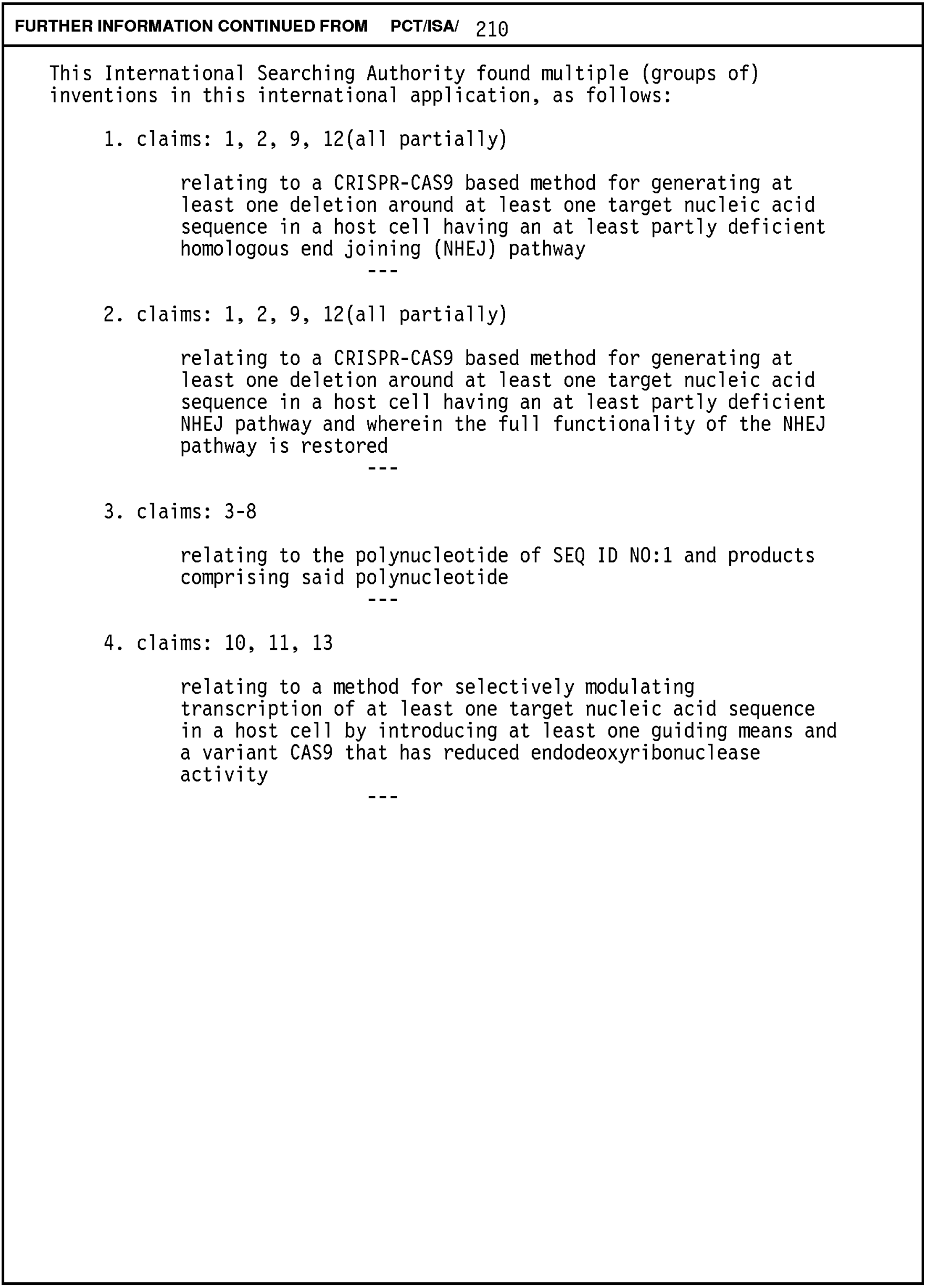

